\title{
DYNAMICS OF MATRIX-FRACTURE COUPLING DURING SHALE GAS PRODUCTION
}

\author{
A Thesis \\ by \\ ASANA WASAKI \\ Submitted to the Office of Graduate and Professional Studies of \\ Texas A\&M University \\ in partial fulfillment of the requirements for the degree of \\ MASTER OF SCIENCE
}

$\begin{array}{ll}\begin{array}{ll}\text { Chair of Committee, } \\ \text { Committee Members, }\end{array} & \begin{array}{l}\text { Ibrahim Yucel Akkutlu } \\ \text { Eduardo Gildin } \\ \text { Marcelo Sanchez }\end{array} \\ \text { Head of Department, } & \text { Alfred Daniel Hill }\end{array}$

August 2015

Major Subject: Petroleum Engineering

Copyright 2015 Asana Wasaki 


\begin{abstract}
In this work, a dynamic permeability model for organic-rich shale matrix is constructed and implemented into a flow simulation to investigate the impact on production. Effective stress and molecular transport effects on the permeability are considered during the flow in the matrix. Using a sector model with a single hydraulicallyinduced fracture, we investigate the dynamic nature of fracture-matrix coupling during the first year of production.
\end{abstract}

The proposed apparent permeability shows improved transport near the fracture due to adsorption capacity of the rock and molecular transport of the adsorbed phase. This fosters drainage and leads eventually to a larger cumulative production. Away from the fracture, however, within the region of depletion, sensitivity to the stress is more pronounced which may impair the local permeability and reduce the production. Overall the shale matrix typically yields more fluid than that with the constant-permeability case.

When the fracture-matrix coupling is considered during the production, an infinite conductivity fracture has negligible effect on the production trends, and that production optimization efforts should focus to considerations to improve the flow rates in the matrix. 


\section{DEDICATION}

To my company, Japan Petroleum Exploration, Co., Ltd., and my dearest husband, Yusuke Wasaki. 


\section{ACKNOWLEDGEMENTS}

I would like to thank my committee chair, Dr. Akkutlu, and my committee members, Dr. Gildin, and Dr. Sanchez for their guidance and support throughout the course of this research. I would also like to thank Dr. Morita for joining the final examination and providing valuable advice, which helped a lot to improve the quality of this work.

Thanks also go to my friends and colleagues and the department faculty and staff for making my time at Texas A\&M University a great experience. Especially, Dr. Dora Restrepo provided a lot of ideas during our discussions. I also want to extend my gratitude to Japan Petroleum Exploration, Co., Ltd., which is the company I work for and they sponsored me throughout the study at Texas A\&M.

Finally, thanks to my mother and father for their encouragement and to my husband for his patience and love. 


\section{NOMENCLATURE}

\begin{tabular}{|c|c|c|}
\hline$A$ & $=$ & Cross section area $\left[\mathrm{m}^{2}\right]$ or $\left[\mathrm{ft}^{2}\right]$ \\
\hline$a$ & $=$ & Aspect ratio [-] \\
\hline$B_{g}$ & $=$ & Formation volume factor for free gas $\left[\mathrm{Rm}^{3} / \mathrm{Sm}^{3}\right]$ or $[\mathrm{rcf} / \mathrm{scf}]$ \\
\hline$C_{\mu}$ & $=$ & $\begin{array}{l}\text { Sorbed-gas concentration in kerogen grain volume }\left[\mathrm{mol} / \mathrm{m}^{3}\right] \text { or } \\
{\left[\mathrm{mol} / \mathrm{ft}^{3}\right]}\end{array}$ \\
\hline$C_{\mu s}$ & $=$ & $\begin{array}{l}\text { Maximum sorbed-gas concentration in kerogen grain volume } \\
{\left[\mathrm{mol} / \mathrm{m}^{3}\right] \text { or }\left[\mathrm{mol} / \mathrm{ft}^{3}\right]}\end{array}$ \\
\hline$C$ & $=$ & Free-gas concentration in pore volume $\left[\mathrm{mol} / \mathrm{m}^{3}\right]$ or $\left[\mathrm{mol} / \mathrm{ft}^{3}\right]$ \\
\hline$c_{g}$ & $=$ & Gas compressibility $[1 / \mathrm{MPa}]$ or $[1 / \mathrm{psi}]$ \\
\hline$c_{p p}$ & $=$ & $\begin{array}{l}\text { Pore compressibility (fixed confining pressure) }[1 / \mathrm{MPa}] \text { or } \\
{[1 / \mathrm{psi}]}\end{array}$ \\
\hline$c_{p c}$ & $=$ & Pore compressibility (fixed pore pressure) $[1 / \mathrm{MPa}]$ or $[1 / \mathrm{psi}]$ \\
\hline$c_{r}$ & $=$ & Rock matrix compressibility [1/MPa] or $[1 / \mathrm{psi}]$ \\
\hline$c_{t}$ & $=$ & Total compressibility $[1 / \mathrm{MPa}]$ or $[1 / \mathrm{psi}]$ \\
\hline$D$ & $=$ & Pore diffusion coefficient $\left[\mathrm{m}^{2} / \mathrm{s}\right]$ \\
\hline$D_{s}$ & $=$ & Diffusion coefficient for sorbed phase transport $\left[\mathrm{m}^{2} / \mathrm{s}\right]$ \\
\hline$E$ & $=$ & Young's modulus [GPa] or [psi] \\
\hline$k_{f}$ & $=$ & Fracture permeability $\left[\mathrm{m}^{2}\right]$ or $[\mathrm{md}]$ \\
\hline$k_{m}$ & $=$ & Matrix permeability $\left[\mathrm{m}^{2}\right]$ or $[\mathrm{md}]$ \\
\hline
\end{tabular}




\begin{tabular}{|c|c|c|}
\hline$K_{n}$ & $=$ & Knudsen number [-] \\
\hline$k_{o}$ & $=$ & Matrix permeability at no effective stress $\left[\mathrm{m}^{2}\right]$ or $[\mathrm{md}]$ \\
\hline$k_{\text {gas }}$ & $=$ & Apparent matric permeability for gas flow $\left[\mathrm{m}^{2}\right]$ or $[\mathrm{md}]$ \\
\hline$M$ & $=$ & Gas molecular weight $[\mathrm{g} / \mathrm{mol}]$ or $[\mathrm{lbm} / \mathrm{mol}]$ \\
\hline$m$ & $=$ & $\begin{array}{l}\text { Associated with geometry of the conductive pore space. }(0 \leq m \\
\leq 1)[-]\end{array}$ \\
\hline$N_{R E}$ & $=$ & Reynolds number [-] \\
\hline$p$ & $=$ & Pore pressure $[\mathrm{MPaA}]$ or $[\mathrm{psia}]$ \\
\hline$p_{c}$ & $=$ & Confining stress $[\mathrm{MPaA}]$ or $[\mathrm{psia}]$ \\
\hline$p_{e}$ & $=$ & Effective stress $[\mathrm{MPaA}]$ or $[\mathrm{psia}]$ \\
\hline$p_{1}$ & $=$ & $\begin{array}{l}\text { Effective stress when slit-shape pores close completely. [MPaA] } \\
\text { or [psia] }\end{array}$ \\
\hline$p_{L}$ & $=$ & Langmuir pressure $[\mathrm{MPaA}]$ or $[\mathrm{psia}]$ \\
\hline$S_{\text {hmin }}$ & $=$ & Minimum horizontal stress $[\mathrm{MPaA}]$ or $[\mathrm{psia}]$ \\
\hline$t$ & $=$ & Production time [sec] \\
\hline$V_{p}$ & $=$ & Pore volume $\left[\mathrm{Rm}^{3}\right]$ or $[\mathrm{rcf}]$ \\
\hline$V_{s L}$ & $=$ & $\begin{array}{l}\text { Langmuir volume, sorbed gas volume per total grain mass } \\
\text { [scf/ton] }\end{array}$ \\
\hline$w$ & $=$ & fracture width $[\mathrm{m}]$ or $[\mathrm{ft}]$ \\
\hline$x$ & $=$ & Spatial coordinate $[\mathrm{m}]$ or $[\mathrm{ft}]$ \\
\hline
\end{tabular}




\section{Greek letters:}

\begin{tabular}{|c|c|c|}
\hline$\alpha$ & $=$ & Biot coefficient or effective stress coefficient [-] \\
\hline$\beta$ & $=$ & Forchheimer coefficient $[1 / \mathrm{m}]$ or $[1 / \mathrm{ft}]$ \\
\hline$\varepsilon$ & $=$ & strain $[-]$ \\
\hline$\varepsilon_{k s}$ & $=$ & $\begin{array}{l}\text { Total organic grain volume per total grain volume }\left[\mathrm{m}^{3} / \mathrm{m}^{3}\right] \text { or } \\
{\left[\mathrm{ft}^{3} / \mathrm{ft}^{3}\right]}\end{array}$ \\
\hline$\Lambda$ & $=$ & Characteristic length of the channel [m] \\
\hline$\lambda$ & $=$ & Mean free path of fluid molecules [m] \\
\hline$\mu$ & $=$ & Gas viscosity $[\mathrm{Pa} \cdot \mathrm{s}]$ or $[\mathrm{cp}]$ \\
\hline$v$ & $=$ & Poisson's ratio [-] \\
\hline$\rho_{\text {grain }}$ & $=$ & Grain density $\left[\mathrm{kg} / \mathrm{m}^{3}\right]$ or $\left[\mathrm{lbm} / \mathrm{ft}^{3}\right]$ \\
\hline$\rho_{s c, g a s}$ & $=$ & Gas density at standard condition $\left[\mathrm{kg} / \mathrm{m}^{3}\right]$ or $\left[\mathrm{lbm} / \mathrm{ft}^{3}\right]$ \\
\hline$\sigma_{o v}$ & $=$ & Overburden stress $[\mathrm{MPaA}]$ or $[\mathrm{psia}]$ \\
\hline$\sigma_{n}$ & $=$ & Normal stress [MPaA] or [psia] \\
\hline$\phi$ & $=$ & Total porosity $\left[\mathrm{m}^{3} / \mathrm{m}^{3}\right]$ or $\left[\mathrm{ft}^{3} / \mathrm{ft}^{3}\right]$ \\
\hline
\end{tabular}




\section{TABLE OF CONTENTS}

Page

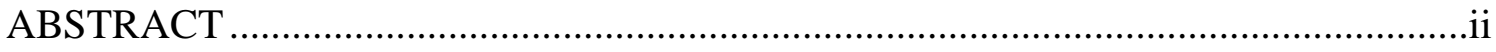

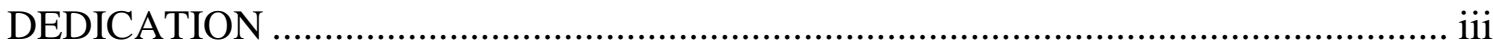

ACKNOWLEDGEMENTS ………………………...................................................iv

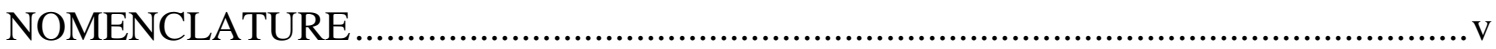

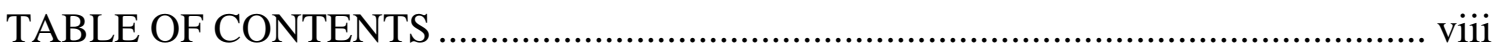

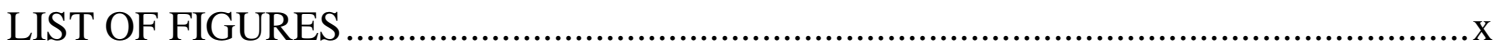

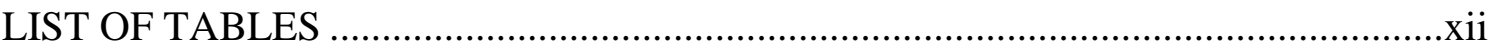

1. INTRODUCTION

1.1 Matrix Flow Models for Organic-Rich Shale ……….............................................

1.2 Hydraulic Fracture Flow Models ……………………........................................

1.3 Scope of the Work and Its Novelty .....................................................................

2. CONTRIBUTION OF MATRIX FLOW TO PRODUCTION.....................................

2.1 Dual-Porosity Nature of the Shale Matrix and the Transport Mechanisms ..............7

2.2 The Organic-Rich Shale Permeability Model .......................................................12

2.3 Analyses on the Apparent Permeability Model.......................................................16

2.4 Description on the In-house Flow Simulator ......................................................... 37

2.5 Impact of the Dynamic Matrix Permeability on Production .....................................47

2.6 Application of the Apparent Permeability Model to a Field Case ..........................54

3. CONTRIBUTION OF FRACTURE FLOW TO PRODUCTION ………………......57

3.1 Threshold Fracture Permeability …………………………………………….....57

3.2 Flow Mechanisms in a Hydraulic Fracture and Its Impact on the Production........67

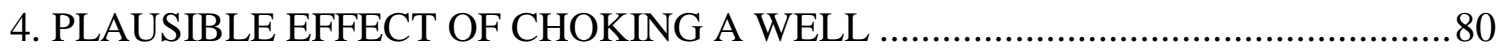

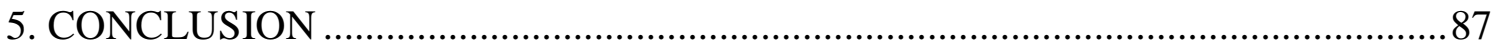

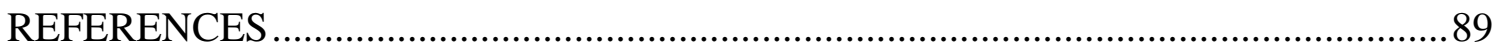

viii 


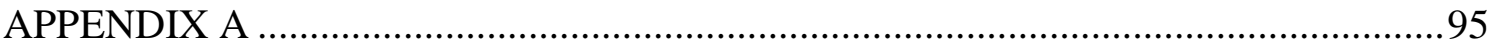

A.1. Derivation of the Sorbed-phase Amount...........................................................95

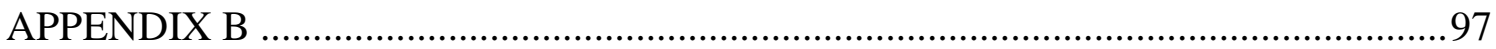

B.1. Derivation of the Apparent Permeability, Equation (2.6) ……………………....97

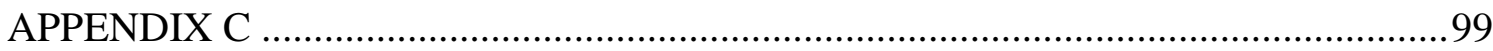

C.1. Derivation of Gangi's Permeability Model ..........................................................99

C.2. Relationship between Permeability of the System and Slit Width .......................100

C.3. Relationship between Net Stress and Slit Width ................................................102 


\section{LIST OF FIGURES}

Page

Figure 2.1. Two-dimensonal SEM image of from Ambrose et al. (2012) ........................ 8

Figure 2.2. Conceptual porosity model for combining multi transport mechanisms .......14

Figure 2.3. Several samples for slit shaped pore orientation .................................. 16

Figure 2.4. Simplified system to build the force balance equation (2.8) ...................... 17

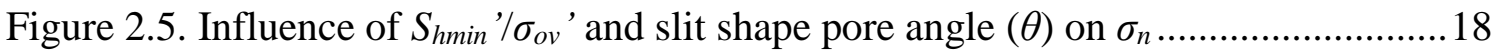

Figure 2.6. Impact of $\theta$ and $v$ on Gangi's permeability model................................20

Figure 2.7. Decomposition of directional permeability ......................................... 21

Figure 2.8. Experimental data matched with Gangi's model .....................................25

Figure 2.9. Analysis of the derived apparent permeability using the cases defined

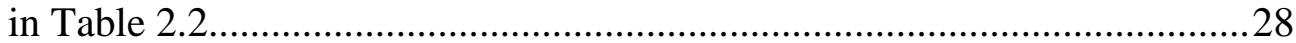

Figure 2.10. Influence of the apparent permeability model to production.....................29

Figure 2.11. Sensitivity of Gangi's model parameters (a) $m$, (b) $p_{1}$, (c) $\alpha$, (d) $D \ldots \ldots \ldots . . .35$

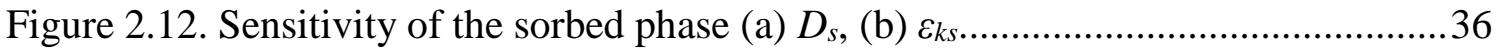

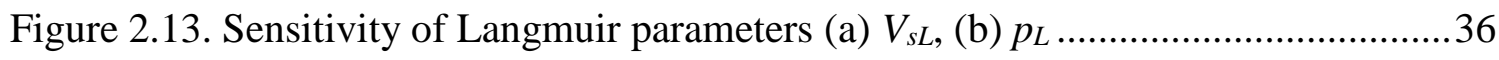

Figure 2.14. A Sector Model with a Single Fracture for 1D/2D flow simulation............38

Figure 2.15. Sample ROCKTAB table for Eclipse input ....................................... 43

Figure 2.16. Comparison between Eclipse and the in-house simulator (Case 1)...........45

Figure 2.17. Comparison between Eclipse and the in-house simulator (Case 2) ...........46

Figure 2.18. Sensitivity analysis of apparent permeability parameters on

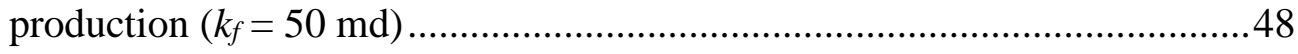

Figure 2.19. Production analysis on a shale reservoir featuring the dynamics in the matrix 
Figure 2.20. Apparent permeability for Formation 1 and Formation $2 \ldots \ldots \ldots \ldots \ldots \ldots \ldots \ldots \ldots . . . .56$

Figure 2.21. Pressure and cumulative production profiles for each formation ................56

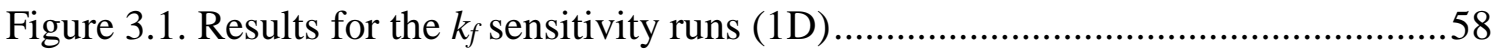

Figure 3.2. Production analysis on a shale reservoir featuring the fracture

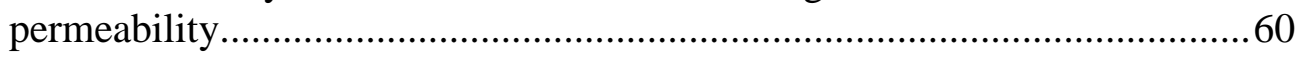

Figure 3.3. Max/Min pressure profile in the fracture .............................................62

Figure 3.4. Sensitivity analysis of apparent permeability parameters on production $\left(k_{f}=5 \mathrm{md}\right.$ colored case compared with $k_{f}=50 \mathrm{md}$ uncolored case)

Figure 3.5. Corresponding apparent matrix permeability to pore pressure distribution after 365 days of production 65

Figure 3.6. Stress sensitive fracture permeability model [modified from Zhang et al. (2014)] 68

Figure 3.7. Idealized creep curve with three regimes [modified from Schwartz and Kolluru (1981)].

Figure 3.8. Stress concentration at (a) joint asperities, (b) proppant.............................70

Figure 3.9. Time deteriorating fracture permeability model $(a=0.057 ; b=1) \ldots \ldots \ldots \ldots . . .71$

Figure 3.11. Fracture permeabilities with Forchheimer effect ..................................76

Figure 3.12. Forchheimer effect on production.................................................... 77

Figure 3.13. Fracture permeability, $k_{f N D}$, and pressure profile ..................................79

Figure 4.1. Choking effect on cumulative production (constant $k_{f}$, dynamic $k_{\text {matrix }}$ ) .......82

Figure 4.2. Stress sensitive fracture permeability models used ................................. 84

Figure 4.3. Effect of choking with stress sensitive fracture permeability ..................... 85

Figure 4.4. Effect of choking with time dependent fracture permeability .....................86 


\section{LIST OF TABLES}

Page

Table 2.1. Parameters used for the base case ............................................................24

Table 2.2. Cases description for quantitative analysis of shale permeability .................27

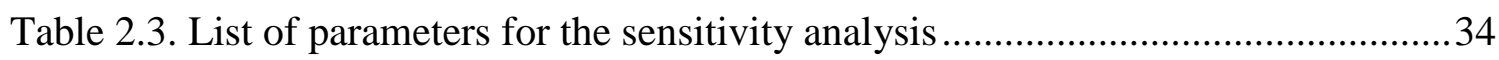

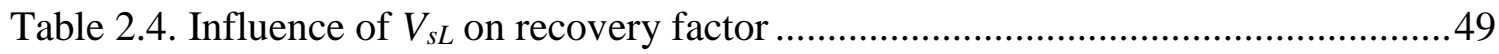

Table 2.5. Effect on uncertainty coming from each flow mechanism in shale

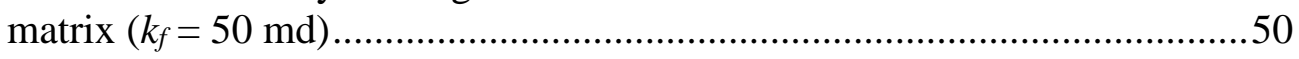

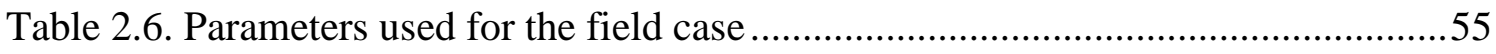

Table 3.1. Effect on uncertainty coming from each flow mechanism in shale

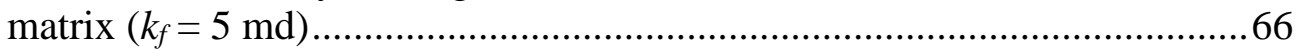




\section{INTRODUCTION*}

A shale well production profile is characterized by a large initial production rate followed by a sharp decline. The rate decline during the first year of production could be as large as $90 \%$ of that recorded during the first days. Because of this production behavior and depending on the economic considerations, the operators may follow a strategy based on continuous drilling and completion of wells in the field. This way, an overall production is maintained in the field, which makes the field operations still profitable. As a consequence, an enormous amount of well data on drilling, well completion and production is coming from the field within a short time span compared with the conventional reservoirs. Commonly, the engineers do not have sufficient time to analyze the production from each well and relate the analyses to well-performance and near-well reservoir characteristics. Consequently, the large production data set is more commonly used with statistical indicators for the ongoing development of the whole field. Understanding the physics underlying the production and using that knowledge in the decision-making process comes at lower priority for the development. However, when our understanding reaches to a maturity and tested in the field, the knowledge could help decrease the uncertainties in production and lead to improved recoveries.

\footnotetext{
"Part of the material in this section is reprinted with permission from "Permeability of Organic-rich Shale" by Wasaki, A., and Akkutlu, I. Y. 2014. SPE-170830 Presented at the SPE Annual Technical Conference and Exhibition, 27-29 October, Amsterdam, The Netherlands. Copyright 2014 by Society of Petroleum Engineers. Further reproduction prohibited without permission.
} 
The large initial production is mainly due to flow of fluids in the fractures. In ratetransient, the early production is often identified by two transient fracture flow regimes: fracture linear flow followed by bi-linear flow. At this early stage of production, contribution of the matrix is negligible. Depending on how large total fracture volume is and how well the complex fracture network has been developed, the fracture flow regimes extend into the production history of the well. Following the fracture flow regimes, the production decline should gradually transient into production from the matrices where the fractures have been embedded. The trend is well-recognized during the rate-transient analysis and it is referred to as the formation linear flow. Although the production rate during the formation linear flow is significantly less than the earlier fracture-dominant production, this regime prevails in the reservoir longer and its contribution to cumulative recoveries from multiple wells over relatively larger times is not insignificant.

The production could be improved when the fluid storage and transport mechanisms in the shale matrix are well-understood. Knowledge on the matrix fluid behavior would improve the quality of the engineering tools, such as the reservoir flow simulators, and decrease the uncertainty in production history-matching. This eventually leads to an improved prediction in well performance and the production optimization.

In this work, permeability models in shale matrix and a hydraulic fracture are revisited to construct a single fracture sector model. There are several mechanisms that could affect the flow capacity in shales. For example, stress sensitivity and molecular transport mechanisms could influence the flow through shale matrix. A new apparent permeability model is proposed in this work with an application to actual formation data. 
By incorporating this model into a flow simulation, influence of each mechanism on the production is analyzed. Further details of the permeability model are discussed in Section 2. Section 2 mainly focuses on flow through shale matrix, however, a well for a shale reservoir typically requires hydraulically induced fractures, and there is significant contribution from the fractures. As for the flow through a hydraulic fracture, the conductivity could change with effective stress or the inertia driven flow condition. The mechanisms that influence the flow in a hydraulic fracture are well-studied by many other researchers, further discussion is in Section 3. By considering the flow mechanisms in the matrix and in a hydraulic fracture, one practical problem of production is investigated. This is an effect of a choking a well, and discussed in Section 4.

In the following, as the introductory section, a brief literature review on shale matrix permeability and hydraulic fracture permeability is presented. At the end of the review, scope of this current work and its novelty is discussed.

\subsection{Matrix Flow Models for Organic-Rich Shale}

Discussion on shale matrix permeability has been active especially within the last decade. It is still mystery to some of us that we are producing petroleum out of a formation previously thought as too tight to produce economically. Efforts continue to reveal the production mechanisms from ultra-tight formations.

The typical flow in a reservoir obeys the Darcy's law. This is applicable for both gas and liquid flow and used extensively in petroleum reservoir systems. However, there are limitations. For example, for gas flow, when the matrix is tight or at low flowing 
pressure, the actual flow could deviate upward from that using Darcy's flow equation. This phenomenon is known as Klinkenberg slip effect and has been recognized since 1941 . Similar phenomenon to Klinkenberg effect is known as Knudsen diffusion and wellstudied in the field of kinetic theory of gases. Several researchers considered that the slippage, Klinkenberg effect or Knudsen diffusion, is occurring in shale matrix and contributing to the production from such a tight formation. For example, Javadpour et al. (2007) and Javadpour (2009) applied the idea of Knudsen diffusion to depict the enhancement of apparent permeability in shale matrix.

Akkutlu and Fathi (2012) looked more into multi-continuum nature of shale matrix, i.e., kerogen and inorganic matrix. Organic-rich shale could bare un-negligible amount of natural gas existing as adsorbed or absorbed phase, which is "sorbed" phase in other word. In their work, they introduced an interesting concept that sorbed phase in kerogen could be mobile, and they showed flux expressions for sorbed phase and free phase (which is not sorbed).

Another interesting work on the flow through shale matrix looks into its sensitivity to effective stress. There is an interesting work done by Heller and Zoback (2013). They conducted a series of permeability measurements on various productive shales and showed that they are sensitive to effective stress.

Interestingly, all these mechanisms that influence the flow are considered almost separately and have not been integrated properly yet. In this work, these mechanisms will be revisited and integrated, starting a discussion from looking at the pore structures in shale matrix. Further detail will be discussed in Section 2. 


\subsection{Hydraulic Fracture Flow Models}

In order to capture the production mechanisms from shales, two different porous media, i.e., shale matrix and a hydraulic fracture, should be considered. In Section 3, as a next step, the flow dynamics in a hydraulic fracture is considered. To make the problem attractable, a simple planar fracture geometry with a rectangular cross section is assumed.

A hydraulic fracture as a porous media has properties more similar to a granular pack with significantly larger porosity when compared to the tight rocks such as shale. Since the flow path in the grains are large, the fluid flow should be considered as a continuum. The complexities that we can account for the flow in the hydraulic fracture are, (i) stress sensitivity, either instantaneous reversible (elastic) response or time dependent irreversible (plastic) response of the fracture, and (ii) the Forchheimer effect. Here, "elastic" is meant by reversible, and "plastic" is meant by irreversible. Elastic behavior is mainly discussed in the production engineering literature, along with figures showing fracture conductivity decline with increasing closure stress [Fredd et al. (2001); Zhang et al. (2014)]. The fracture conductivity changing with time is associated with a phenomenon called "proppant embedment," and it is considered as time dependent plastic behavior to the stress. Fracture conductivity changing with time is also discussed in Wen et al. (2007) and Zhang et al. (2014). The other mechanism on the flow, the Forchheimer effect, is similar to the skin effect which depends on the pore pressure and the flow rate. This phenomenon in a hydraulic fracture is discussed by Miskimins et al. (2005) and Amini and Valkó (2010). In this work, these mechanisms will be revisited and considered one by one in later Section 3. 


\subsection{Scope of the Work and Its Novelty}

The ultimate goal of this research is to improve understanding on production from organic-rich shale. This work starts from visualizing the rock at a fine scale and constructing a conceptual model for the fluid transport through the matrix. The conceptual model will add modification to the storage mechanisms and flow mechanisms. This model will be used to develop an apparent permeability model, and will be applied to an in-house flow simulator. Shale matrix solely cannot deliver petroleum from the reservoir to a wellbore; a hydraulic fracture is necessary for production. The mechanisms previously discussed in this section will be considered to develop the coupled model of shale matrix and a hydraulic fracture. The main contribution of this work is in proposing a new permeability model for shale matrix, as well as showing sample applications of the permeability model.

The limitations of this work are the following. (i) It is limited to a single phase; (ii) although current trend is to fully-couple the mass balance equation for fluid and the momentum balance equation for geomechanics, the in-house simulator used in this work just considers the mass balance equation for fluid and does not consider the momentum balance equation for geomechanics. Therefore, the contribution of rock deformation on pore pressure is not taken into account. 


\section{CONTRIBUTION OF MATRIX FLOW TO PRODUCTION*}

\subsection{Dual-Porosity Nature of the Shale Matrix and the Transport Mechanisms}

Taking a look at FIB-SEM images such as the one shown in Figure 2.1 helps a lot to consider the flow through shale matrix. Clearly, the image shows a dual porosity system with organic round pores and slit-shape pores. The sizes and shapes of these pores are quite different and bring in a multi-scale feature to the matrix which is important for fluid storage and transport. Similar discussions on pore structures can be found in Palciauskas and Domenico (1980) and Loucks et al. (2009, 2012). Round-shape organic pores are developed as a result of thermal maturation and conversion of kerogen. And slit-shape pores are developed as a result of cracking caused by fluid pressures in excess of hydrostatic, and this could exist both in organic/inorganic material. The organic material has a strong affinity (relatively strong molecular interaction) for the hydrocarbons and the organic nanopores have large specific surface area associated with the pore walls. Hence, the organic pores are the ideal places for massive storage of hydrocarbons as adsorbed and dissolved fluids (which we call as "sorption" later on). The other storage mechanism (or the state of fluid molecules) is the conventional "free" fluid. This observation and consideration on pore structures and storage mechanisms makes up the basis of our discussions on the fluid transport in the following pages.

\footnotetext{
* Part of the material in this section is reprinted with permission from "Permeability of Organic-rich Shale" by Wasaki, A., and Akkutlu, I. Y. 2014. SPE-170830 Presented at the SPE Annual Technical Conference and Exhibition, 27-29 October, Amsterdam, The Netherlands. Copyright 2014 by Society of Petroleum Engineers. Further reproduction prohibited without permission.
} 


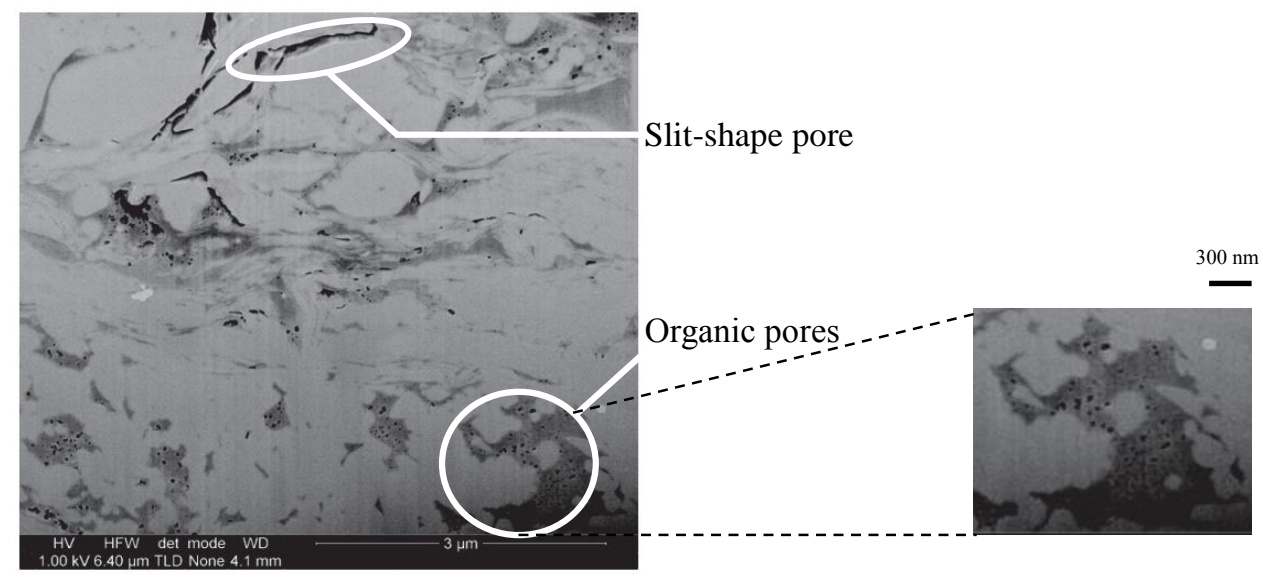

Figure 2.1. Two-dimensonal SEM image of from Ambrose et al. (2012)

In order to explain the flow regimes of free gas, Knudsen Number, $K_{n}(=\lambda / \Lambda)$, is often used. Here $\lambda$ is mean free path of the fluid molecule and $\Lambda$ is the pore diameter. This is a dimensionless number that represents a ratio of fluid-fluid molecules collision distance to fluid-wall molecules collision distance [Karniadakis et al. (2005)]. Although $K_{n}$ has several parameters to control its value, a common discussion under the reservoir conditions includes the width of the flow path, i.e., the capillary diameter or channel size. The larger the width, the smaller $K_{n}$. Let us follow this tradition and explain the possible flow regimes in the shale matrix. Consider the transport of the fluid which exist as free gas (not sorbed) in the organic and inorganic pores. In the slit-shape pore-network, the average channel size is large such that $K_{n} \leq 0.01$. This is when the classical continuum treatment of fluid flow is valid. Thus laminar (or viscous) flow can be used for the description of free gas transport in this pore network. In reservoir engineering, the laminar 
flow in porous media is represented by Darcy's law. Hence, using the same approach, we should define a permeability for the slit-shape pore-network of the shale matrix.

With regards to the flow in matrix, there are many experimental observations suggesting that the absolute permeability of rocks changes with the confining stress. Kwon et al. (2004), and Heller and Zoback (2013) have previously showed the stress-dependence of shale samples in the laboratory. Although several models have been proposed to capture the stress-dependence of the matrix permeability, we have rather limited choices for a matrix pore-network with slit-shaped pores: Gangi (1978) and Walsh (1981) models. Kwon et al. (2004) have previously showed that Gangi's model could represent the stressdependent permeability of the shale matrix. Here, we use the model to describe the stressdependence of the slit-shape pore network:

$$
k_{m}=k_{o}\left(1-\left(\frac{p_{c}-\alpha p}{p_{1}}\right)^{m}\right)^{3}
$$

In Equation (2.1) $p_{c^{-}} \alpha \cdot p$ is the effective stress on the matrix. $k_{o}$ is the permeability at zero effective stress. $p$ is the pore pressure and we often refer to it as the reservoir pressure during the production. $p_{l}$ is the effective stress when the slit-shape pores are closed completely, i.e., when $k_{m}=0$. With larger $p_{l}$, the slit-shape pores become less sensitive to the effective stress because it becomes more difficult to close the pores. An extreme case is when $p_{l}$ is infinitely large. In this case, $k_{m} \rightarrow k_{o}$ and the slit-shape pores become totally insensitive to the effective stress. Finally, the exponent $m$ is associated with the surface roughness of the slit-shape pores. In the original paper, Gangi (1978) considers that the pore surfaces could be rough and they can be conceptually modelled as a bed of 
nails supporting the pore walls (Figure C.1). In the model, $m$ represents a distribution of the height of nails. If the nails heights are close to uniform with only a few shorter nails, then $m$ is close to unity. If there is significant variability in the heights, then the surface roughness increases and $m$ takes values approaching zero. Further explanation on Gangi's model and the derivation of Equation (2.1) is given in Appendix C.

Conceptually, we have two different pore structures. One is round-shape pores and the other is slit-shape pores. In terms of size, we assume the latter is much larger according to the observation from SEM images. We assume the slit-shape pores are stress sensitive, on the other hand, the stress sensitivity for the round-shape pores is negligible. Stress sensitivity of each pore can be analyzed by looking at the aspect ratio of its cross section. According to Zimmerman (1991), Pore compressibility under fixed confining pressure, $c_{p p}=1 / V_{p}\left(\partial V_{p} / \partial p\right)_{p_{c}=\text { const. }}$, has a following relationship:

$$
c_{p p}=c_{p c}-c_{r}
$$

Here, $V_{p}$ is pore volume, $c_{p c}$ is pore compressibility under fixed pore pressure, $c_{p c}=-1 / V_{p}\left(\partial V_{p} / \partial p_{c}\right)_{p=\text { const. }}$, and $c_{r}$ is the compressibility of the rock matrix. Consider a rock including pore with different aspect ratio, $a$. Then $c_{r}$ will be the same, on the other hand, $c_{p c}$ changes with aspect ratios. The relation between the aspect ratio and $c_{p c}$ is given by the following equation:

$$
c_{p c}=\frac{2\left(1-v^{2}\right)}{E}\left[a+\frac{1}{a}\right]
$$

$v$ is Poisson's ratio and $E$ is Youngs' modulus. For example, if an aspect ratio of slit-shape pore or organic round pore is taken from Figure 2.1, the aspect ratio for slit- 
shape pore is around 0.1. On the other hand, organic pores have the aspect ratio close to 1. This contrast creates $c_{p c}$ value one order of magnitude higher for the slit-shape pore. Therefore, it should be easier to change the cross-sectional area for slit-shape pores compared with organic pores, and stress sensitive permeability mainly comes from cross sectional change in slit-shape pores.

In the organic round-shape pore network (or some part of inorganic pore network with narrower flow path), on the other hand, the capillary size is significantly reduced. This leads to an increase in $K_{n}$. The transport of "free" fluid now experiences significantly less number of fluid molecules in the capillary. With the decreasing capillary size, the fluid in the pores gradually becomes difficult to treat as a continuum. It gradually becomes difficult to use macroscopic properties such as viscosity, and becomes more of a collection of molecules. Consequently, the transport is expected to experience a shift from free viscous flow to free molecular transport, i.e., Knudsen diffusion. Several authors have previously worked on the identification of Knudsen diffusion due to increased fluid-wall molecular interactions at larger $K_{n}$. Recent work by Fathi et al. (2012) showed that Knudsen diffusion is not likely to develop in the organic (and inorganic) capillaries due to large pressures experienced in the shale reservoirs. The resource shales are over-pressured and the production is typically held at flowing bottomhole condition that is likely to be larger than 500 psi. Under such high pressures, the wall effects on the mass flux of the free gas is insignificant. Hence, we do not consider that Knudsen diffusion contributes to the mass flux of the free gas. Instead, we introduce pore diffusion which is expressed in the form of Fickian diffusion. Because when these pores are small enough, the velocity 
profile associated with viscous flow may not develop. Consequently, pore diffusion may become the dominant transport mechanism. The mass flux for pore diffusion is proportional to free fluid concentration gradient and its diffusion coefficient is different from that of free molecular transport.

As for the sorbed phase transport in the organic material, there are conflicting arguments in the literature. Although some literature treats the sorbed-phase as immobile, for example Sakhaee-Pour and Bryant (2012), others argued that the sorbed phase can be driven by the sorbed-phase density, or concentration, gradient [Do (1998)] and could be mobile under the reservoir conditions [Fathi and Akkutlu (2009), Kang et al. (2011), Akkutlu and Fathi (2012), and Riewchotisakul (2015)]. This paper follows the latter position and consider the sorbed-phase transport. We use Langmuir isotherm to represent the sorbed phase. The Langmuir model is based on two assumptions. One is that adsorption and desorption of the molecules are under equilibrium. Further description can be found in Appendix A. The other assumption is that the sorbed-phase can be treated as mono-layer adsorption. The sorbed-phase mass flux obeys Fickian diffusion, which is the commonly used expression for sorbed phase transport.

\subsection{The Organic-Rich Shale Permeability Model}

Based on the discussions on multi-scale transport, in Figure 2.2 we propose a simple conceptual model for fluid transport in organic rich shale. In a sense, this approach is similar to conventional treatment of the two phase flow in porous media: each phase follows its own path obeying its own transport mechanisms. We consider a parallel flow 
of free gas phase and sorbed phase. We also assume that pore pressure is in equilibrium within the specified bulk volume and it is the same in organic and inorganic pores. A mass balance equation is built, in terms of moles of gas per unit bulk volume of rock, as a summation of the mass balance equations for the free gas phase and the sorbed phase, Equation (2.4).

$$
\begin{aligned}
\frac{\partial}{\partial t}\left[\varepsilon_{k s}(1-\phi) C_{\mu}\right]+\frac{\partial}{\partial t}[\phi C] & \\
= & \frac{\partial}{\partial x}\left(D_{s} \frac{\partial C_{\mu}}{\partial x}\right)+\frac{\partial}{\partial x}\left(D \frac{\partial C}{\partial x}\right)+\frac{\partial}{\partial x}\left(C \frac{k_{m}}{\mu} \frac{\partial p}{\partial x}\right)
\end{aligned}
$$

Here, $x$ - $t$ are the space-time coordinates. $C(x, t)$ represents the moles of free gas per unit pore volume. Using the compressibility equation of state, the free gas amount is related to pore pressure using $C=p / z R T$. $C_{\mu}(x, t)$ represents the amounts of sorbed-phase in the organic pores in terms of moles per kerogen grain volume. It can be expressed in terms of the pore pressure using the Langmuir model $C_{\mu}=C_{\mu s} \cdot p /\left(p+p_{L}\right)$, where $p_{L}$ is the Langmuir pressure and $C_{\mu s}$ is the maximum sorption capacity of the shale matrix due to presence of the organic material. Derivation of $C_{\mu}$ is shown in Appendix A. $\phi$ is the total interconnected matrix porosity in shale and includes to pore volume contributions of the organic and inorganic pores. $\varepsilon_{k s}$ is the total organic content in terms of organic grain volume per total grain volume. $\varepsilon_{k s}(1-\phi)$ is the fractional kerogen grain volume. Essentially, the left-hand-side of Equation (2.4) shows the total amount of gas: the first term being the amount stored as the sorbed phase and the second term the amount stored as free gas. 


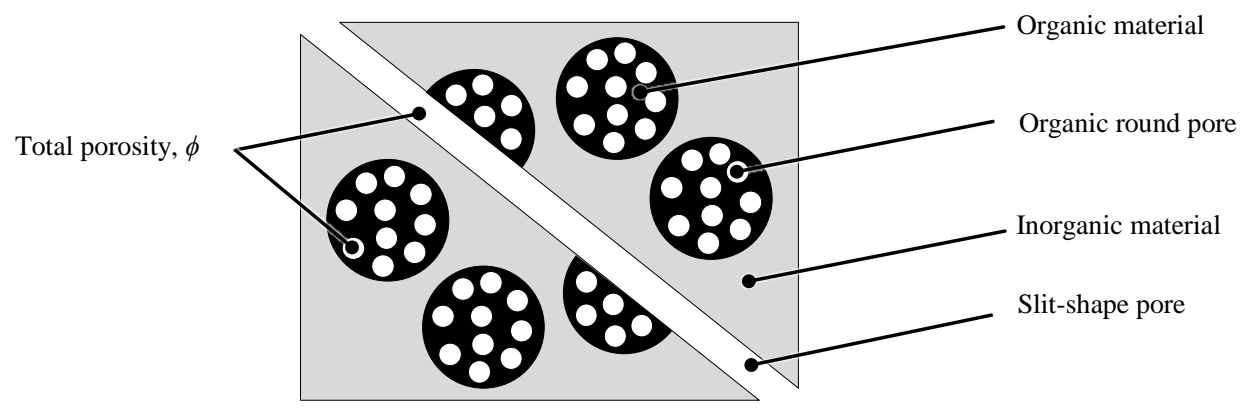

Figure 2.2. Conceptual porosity model for combining multi transport mechanisms

Turning to the right-hand-side of Equation (2.4), first term shows the mass flux due to the sorbed-phase transport, and $D_{s}$ is the diffusion coefficient. The second term shows the free gas mass flux due to pore diffusion, and $D$ is pore diffusion coefficient. The third term accounts for the viscous flow in the slit-shape pore network, and $k_{m}$ is the absolute permeability mainly controlled by slit-shape pores as defined in Equation (2.1), and $\mu$ the dynamic gas viscosity. In this way, we could take care of viscous flow in slitshape pores as well as pore diffusion occurring in the organic pores.

In summary, we consider total pore volume as a place for free gas storage, and storage due to sorption is associated with kerogen grain volume. As for transport mechanisms, we assume viscous flow with stress dependent permeability and pore diffusion for free gas phase, and the Fick's law type of diffusion for sorbed phase. Here, note that pore pressure, $p$, shown in Equation (2.6) is averaged pressure for a given bulk rock volume.

Now, we combine the mass flux terms on the right-hand-side of Equation (2.4), extract the terms that have the same dimension as the permeability and define it as an 
apparent permeability. As we manipulate the right-hand-side, we introduced the following Equation (2.5) for $C_{\mu s}$ :

$$
C_{\mu s}=\frac{V_{s L} \rho_{s c, g a s} \rho_{g r a i n}}{\varepsilon_{k s} M}
$$

$V_{s L}$ is the so called Langmuir volume, which shows the maximum sorbed gas volume converted into standard condition per unit total grain mass, $\rho_{s c, g a s}$ the gas density at standard condition, $\rho_{\text {grain }}$ the grain density, and $M$ the gas molecular weight. Equation (2.6) is the final outcome of the apparent shale matrix permeability. For further details of the derivation, please refer to Appendix B.

$$
k_{g a s}=k_{m}+\mu D c_{g}+\mu D_{s} \frac{V_{s L} \rho_{\text {grain }} B_{g}}{\varepsilon_{k s}} \frac{p_{L}}{\left(p+p_{L}\right)^{2}}
$$

In Equation (2.6), we observe the contribution of each introduced transport mechanism. $k_{m}$ includes stress-dependent permeability of the slit-shape pore-network as described in Equation (2.1), the second term is the contribution of free gas pore diffusion both in the organic and inorganic pore networks. Finally, the third term reflects the contribution of the sorbed phase transport through the organic pore-network. In essence, the last two terms include deviation from the Darcy flow in the organic-rich shale matrix.

Equation (2.6) is derived for the gas transport in organic-rich shale. When the single phase flow is due to liquid hydrocarbons (oil), new considerations should come into play. Firstly, the solid-liquid interactions associated with the oil molecules residing in kerogen are significantly larger in comparison to the natural gas case. This is due to presence of much larger attractive forces by the walls in the presence of liquid [Bui and Akkutlu (2015)]. Hence, one needs to follow the conventional treatment and do not expect 
deviation from Darcy's permeability for free liquid flow (not sorbed). This leaves us with an apparent permeability for liquid as following Equation (2.7):

$$
k_{\text {liquid }}=k_{m}
$$

\subsection{Analyses on the Apparent Permeability Model}

\subsubsection{Consideration on Effective Stress for Gangi's Model}

Here, Gangi's permeability model given in Equation (2.1) is considered. In the original publication, the confining stress, $p_{c}$, is isotropic. However, the stress orientation in an actual reservoir is most likely to be anisotropic. Here, in this section, effective stress to feed into Equation (2.1) is considered and discussed in depth.

(a) $90^{\circ}$ from $S_{h \min }$,

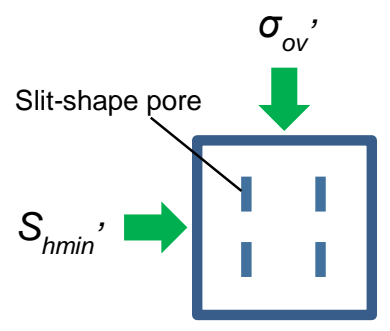

(b) $\theta$ from $S_{\text {hmin }}$ '

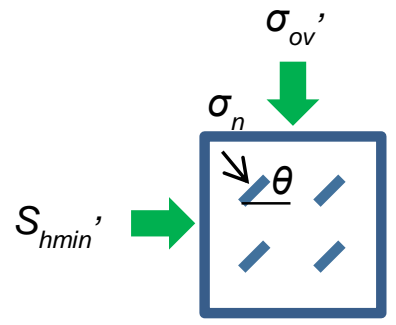

(c) $0^{\circ}$ from $S_{\text {hmin }}$,

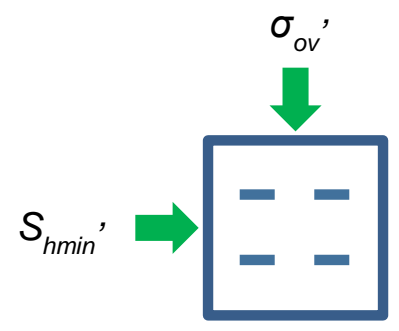

Figure 2.3. Several samples for slit shaped pore orientation 
One simple way to start thinking about this problem is to take a cross section in vertical direction and assume all the slit-shape pores are aligned uniformly as shown in Figure 2.3. Vertical stress is the overburden stress, $\sigma_{o v}$. Horizontal stress, $S_{h m i n}$, also exists, and its magnitude depends on the rock properties. Assuming no shear stress, then that will allow us to estimate the normal stress, $\sigma_{n}$, to the slit easily, as shown in Equation (2.8).

In Figure 2.3(b), the slit is $\theta$ tilted from horizontal direction. In that case, the normal stress $\left(\sigma_{n}\right)$ to the slit is a combined stress of $\sigma_{o v}$ ' and $S_{\text {hmin }}$, and could be written as shown in Equation (2.9). Here, $\sigma_{o v}$ ' is effective overburden stress, $\sigma_{o v}-\alpha \cdot p$, and $S_{h m i n}$ ' is effective minimum horizontal stress, $S_{h m i n}-\alpha \cdot p$. Equation (2.9) is derived by building a force balance equation (2.8) between $\sigma_{o v}{ }^{\prime}, S_{h m i n}$ ' and $\sigma_{n}$ assuming a simplified system shown in Figure 2.4. First, extract a triangular portion including a slit at one of the edges. $\sigma_{o v}$ ' and $S_{h \min }$ ' split into two elements. One is parallel to $\sigma_{n}$ and the other is orthogonal. With the elements parallel to $\sigma_{n}$, Equation (2.8) is derived followed by Equation (2.9).

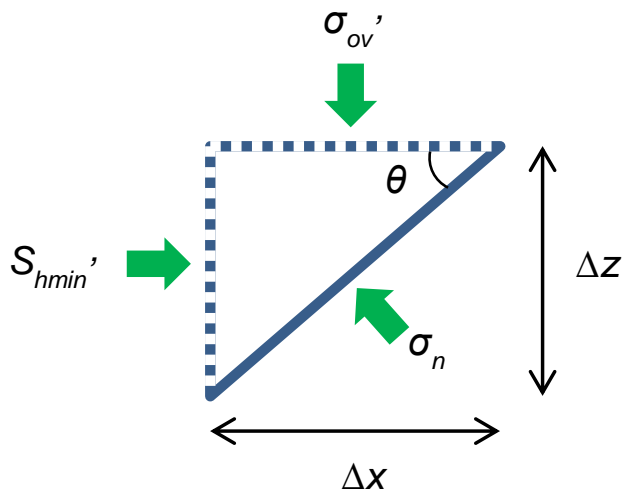

Figure 2.4. Simplified system to build the force balance equation (2.8) 


$$
\begin{gathered}
\sqrt{(\Delta x)^{2}+(\Delta z)^{2}} \sigma_{n}=\sigma_{o v}^{\prime} \Delta x \cos (\theta)-S_{h m i n}^{\prime} \Delta z \sin (\theta) \\
\sigma_{n}=\frac{\sigma_{o v}^{\prime}-S_{h m i n}^{\prime}}{2} \cos (2 \theta)+\frac{\sigma_{o v}^{\prime}+S_{h m i n}^{\prime}}{2} \ldots \ldots \ldots
\end{gathered}
$$

By dividing Equation (2.9) by $\sigma_{o v}$, the following equation can be derived. This ratio is a function of $\theta$ and $S_{h m i n}{ }^{\prime} / \sigma_{o v}$ '. This is the function shown in Figure 2.5 .

$$
\sigma_{n} / \sigma_{o v}^{\prime}=\frac{1}{2}\left(1-\frac{S_{h \min }^{\prime}}{\sigma_{o v}^{\prime}}\right) \cos (2 \theta)+\frac{1}{2}\left(1+\frac{S_{h \min }^{\prime}}{\sigma_{o v}^{\prime}}\right)
$$

$S_{h \min }{ }^{\prime} / \sigma_{o v}{ }^{\prime}=1 / 3$ in Figure 2.5 is a case when a typical value of Poisson's ratio, $v=$ 0.25 is used. $S_{h m i n}{ }^{\prime} / \sigma_{o v}{ }^{\prime}=0,1$ show the extreme cases. The former represents the case $S_{h \min }{ }^{\prime}=0$, and the latter shows the case $S_{h m i n}{ }^{\prime}=\sigma_{o v}{ }^{\prime}$. The figure shows that the maximum confining stress is $\sigma_{o v}$ ' when $\theta=0$, and the minimum is $S_{h m i n}$ ' when $\theta=\pi / 2$.

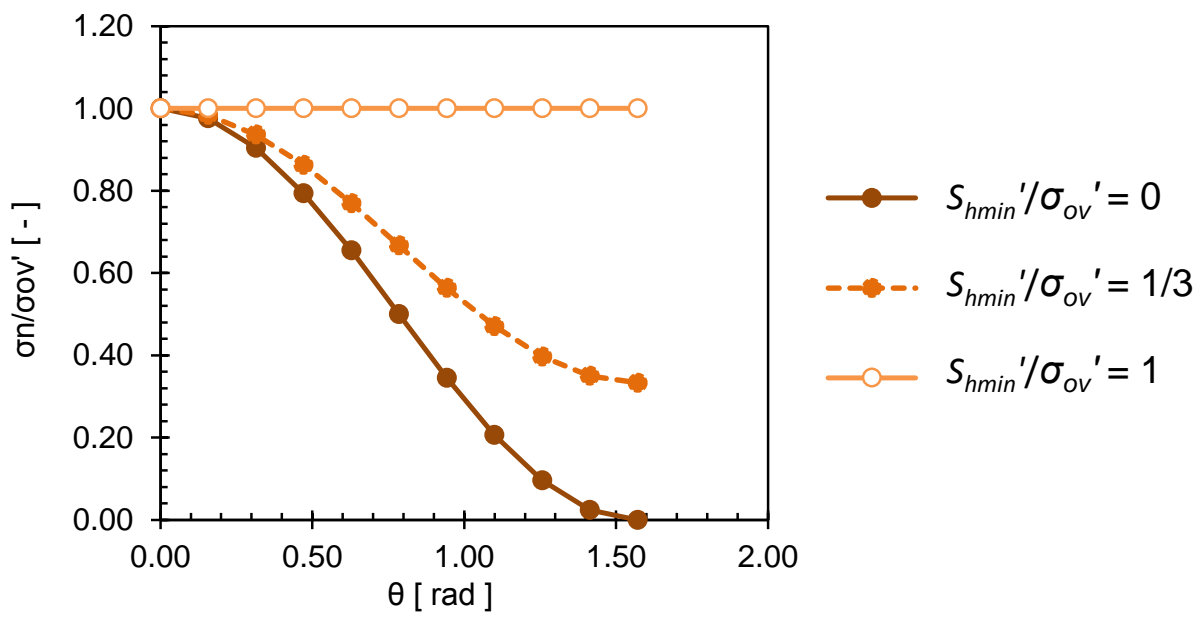

Figure 2.5. Influence of $S_{h m i n} / \sigma_{o v}$ ' and slit shape pore angle $(\theta)$ on $\sigma_{n}$ 
If $\sigma_{o v}{ }^{\prime}, S_{h m i n}$, and $\theta$ are given, assuming all pores are aligned in the same direction, $\sigma_{n}$ can be calculated by Equation (2.9). The simplest relation between effective overburden stress, $\sigma_{o v}{ }^{\prime}$, and effective horizontal stress, $S_{h m i n}$ ', is in Equation (2.11). $v$ is Poisson's ratio. This relation is derived by assuming no deformation in horizontal direction. By applying the definition of effective stress, Equation (2.11) is reshaped to obtain Equation (2.12).

$$
\begin{gathered}
S_{h \min }^{\prime}=\frac{v}{1-v} \sigma_{o v}^{\prime} \\
\Leftrightarrow\left(S_{\text {hmin }}-\alpha p\right)=\frac{v}{1-v}\left(\sigma_{o v}-\alpha p\right) \\
S_{\text {hmin }}=\frac{v}{1-v} \sigma_{o v}+\left(\frac{1-2 v}{1-v}\right) \alpha p
\end{gathered}
$$

$S_{h m i n}$ shown in Equation (2.12) is pore pressure dependent. Combining Equation (2.9) and (2.11) gives Equation (2.13). Now $\theta$ and $v$ are added to Equation (2.1).

$$
k_{x^{\prime} x^{\prime}}=k_{o}\left[1-\left\{\frac{(1-2 v) \cos (2 \theta)+1}{2(1-v) p_{1}}\left(\sigma_{o v}-\alpha p\right)\right\}^{m}\right]^{3} \ldots \ldots \ldots
$$

Figure 2.6 shows how these additional parameters affect the stress sensitive permeability, $k_{x^{\prime} x^{\prime}}$. In Figure 2.6(a), $\theta$ in Equation (2.13) is shifted from 0 to $\pi / 2$ under constant $v$. In this case, the slit-shape pore first parallel to horizontal ends up being perpendicular to the original alignment. As $\theta$ becomes larger, $\sigma_{n}$ decreases as is seen from Equation (2.9), which leads to upward shift in permeability. Figure 2.6(b) shows the case when $v$ is shifted from 0 to 0.5 with fixed $\theta . v=0$ is the case when the rock doesn't have any resistance to maintain its volume. Therefore $S_{h \min }{ }^{\prime}=0$ for any $\sigma_{o v}{ }^{\prime}$ as seen from Equation (2.11), and thus $\sigma_{n}=0$. This is the reason why it seems there is no sensitivity to 
the effective stress. The case when $v=0.5$ is when the rock has the largest resistance to its volume change, and therefore $\sigma_{n}$ becomes the largest.

(a) Fixed $v=0.25 ; \theta$ changed from 0 to $\pi / 2$

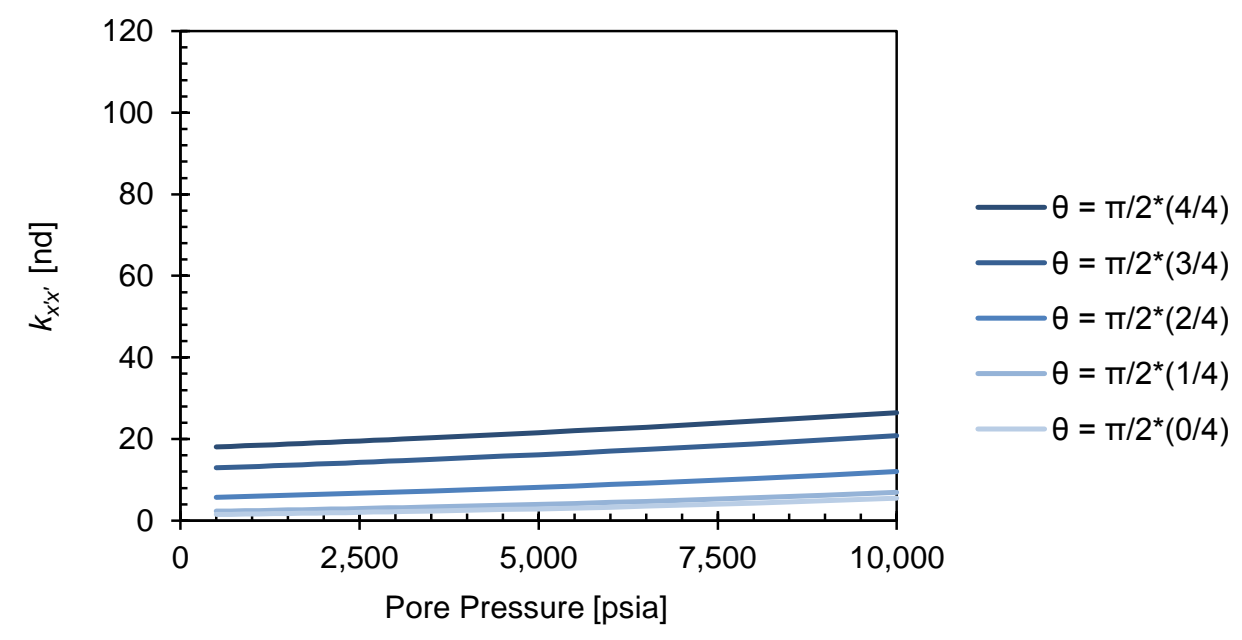

(b) $v$ changed from 0 to 0.5 ; Fixed $\theta=\pi / 2$

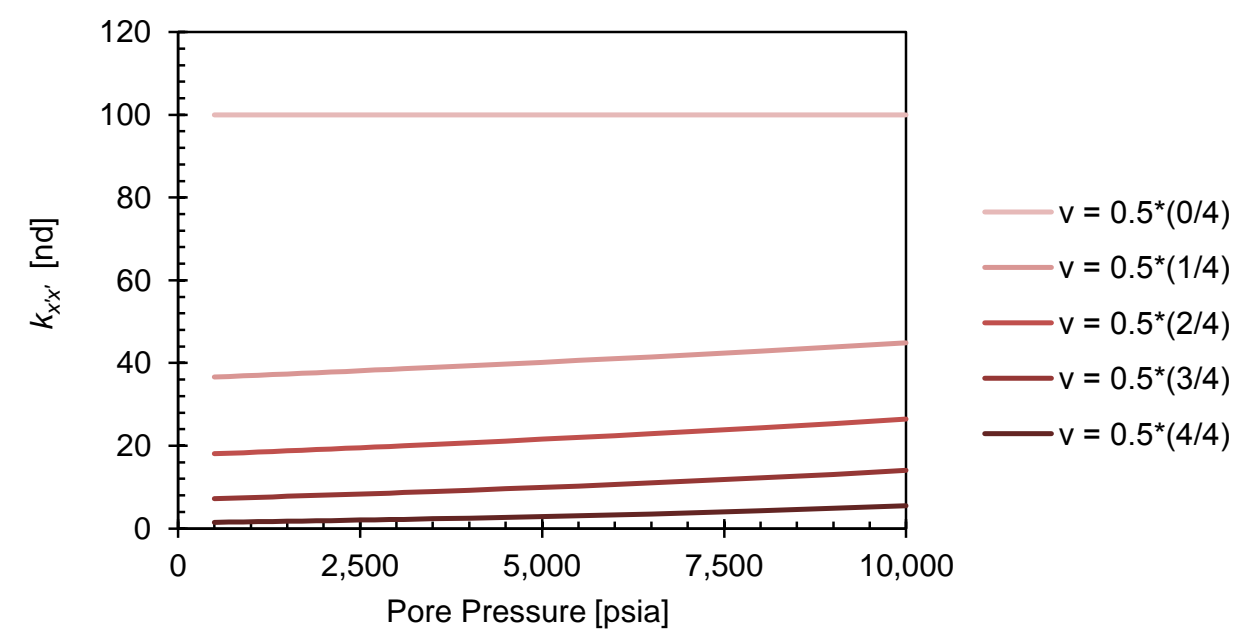

Figure 2.6. Impact of $\theta$ and $v$ on Gangi's permeability model 
One thing to keep in mind here is that Equation (2.13) shows directional permeability along the extent of the slit. If the slit is not aligned in the direction parallel to horizontal, the permeability shown in Equation (2.13) has to be decomposed into horizontal component and vertical component to feed into any commercial simulator. Figure 2.7 explains this graphically. $k_{x}^{\prime} x^{\prime}$ in Figure 2.7(b) corresponds to the permeability in Equation (2.13), which is tilted $\theta$ from horizontal direction. This permeability is good for the $x^{\prime}-z^{\prime}$ coordinate shown in Figure 2.7(a). However, in many case, a simulator employs $x$-z coordinate in Figure 2.7(a), therefore $k_{z z}$ and $k_{x x}$ in Figure 2.7(b) is more favored as inputs for a simulator. In the $x^{\prime}-z^{\prime}$ coordinate, second-order permeability tensor in $2 \mathrm{D}$ is expressed in Equation (2.14) with zero off-diagonal elements, and $k_{x^{\prime} x}{ }^{\prime}$ corresponds to the permeability in Equation (2.13).

$$
\mathbf{k}^{\prime}=\left[\begin{array}{cc}
k_{x^{\prime} x^{\prime}} & 0 \\
0 & k_{z^{\prime} z^{\prime}}
\end{array}\right]
$$

(a) Coordinate rotation

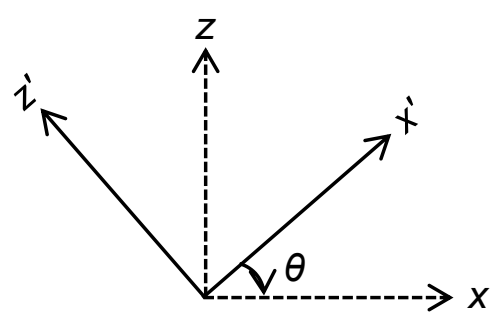

(b) Decomposing permeability

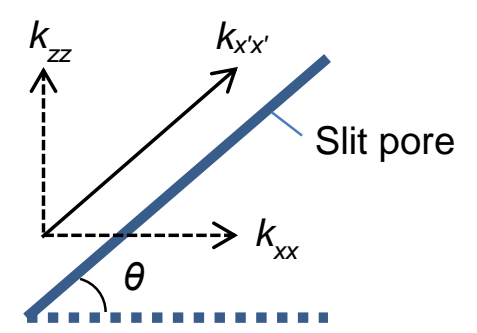

Figure 2.7. Decomposition of directional permeability 
Since $\left(x^{\prime}, z^{\prime}\right)$ can be expressed by $(x, z)$ as shown in Equation (2.15), the secondorder permeability tensor, $\mathbf{k}$, can be transformed for the $x$ - $z$ coordinate by applying rotation [see Equation (2.16) and (2.17)].

$$
\begin{array}{r}
{\left[\begin{array}{l}
x \\
z
\end{array}\right]=\left[\begin{array}{cc}
\cos \theta & -\sin \theta \\
\sin \theta & \cos \theta
\end{array}\right]\left[\begin{array}{l}
x^{\prime} \\
z^{\prime}
\end{array}\right] \ldots} \\
\{\mathrm{k}\}_{\mathrm{ij}}=\boldsymbol{n}_{i} \cdot \mathbf{k}^{\prime} \cdot \boldsymbol{n}_{j} \quad(i, j=1,2)
\end{array}
$$

Where,

$$
\boldsymbol{n}_{1}=\left[\begin{array}{c}
\cos \theta \\
-\sin \theta
\end{array}\right] ; \boldsymbol{n}_{2}=\left[\begin{array}{c}
\sin \theta \\
\cos \theta
\end{array}\right]
$$

This rotation described in Equation (2.16) and (2.17) will give the following transformed permeability tensor, $\mathbf{k}$ in Equation (2.18).

$$
\mathbf{k}=\left[\begin{array}{cc}
\left(k_{x^{\prime} x^{\prime}}-k_{z^{\prime} z^{\prime}}\right) \cos ^{2} \theta+k_{z^{\prime} z^{\prime}} & \left(k_{x^{\prime} x^{\prime}}-k_{z^{\prime} z^{\prime}}\right) \cos \theta \sin \theta \\
\left(k_{x^{\prime} x^{\prime}}-k_{z^{\prime} z^{\prime}}\right) \cos \theta \sin \theta & \left(k_{z^{\prime} z^{\prime}}-k_{x^{\prime} x^{\prime}}\right) \cos ^{2} \theta+k_{x^{\prime} x^{\prime}}
\end{array}\right]
$$

However, in this work, we only consider the case when $\theta=0$. Therefore Equation (2.14) will become the permeability tensor of interest. Moreover, vertical flow will not be discussed, therefore the stress sensitive permeability appears in this work remains same as Equation (2.1), which is the original Gangi's permeability model with $p_{c}$ equals to overburden stress, $\sigma_{o v}$. 


\subsubsection{Impact of Each Mechanism in the Derived Apparent Permeability}

As mentioned previously, we see three terms in the apparent gas permeability. In this section, in order to measure the impact of each component, four cases are introduced in Table 2.2. The numerical outcome of these cases as they change with the pore pressure are shown in Figure 2.9. Data shown in Table 2.1 are used as inputs for the calculations.

Sample data shown in Table 2.1 is prepared using on a combination of data sources of gas producing shale. The reservoir properties of temperature, initial pore pressure and porosity are taken from Eagle Ford data presented in Meyer et al. (2010). Pore compressibility is a typical value. As for the sorption properties, the Langmuir volume and pressure are chosen as average values from Santos and Akkutlu (2013). $\varepsilon_{k s}$ can be estimated by taking a ratio of grain density and kerogen density and multiply it by TOC (Total Organic Carbon). The gas in the system is considered as pure methane for simplicity. Grain density, $\rho_{\text {grain }}$ is a typical value, and bulk rock density, $\rho_{b u l k}$, is from Ambrose et al. (2012).

As for the parameters for the stress-dependent permeability model, $p_{1}$ is obtained by matching with the data from Heller and Zoback (2013). The sample number Eagle Ford 127 is used. Since they used helium for permeability measurement, an apparent permeability model without sorbed phase transport term, Equation (2.6), is used for matching. This apparent permeability model can be reshaped as shown in Equation (2.19). By plotting the right-hand-side over natural logarithm of effective stress, a set of Gangi's model parameters $\left(k_{o}, p_{1}\right.$, and $\left.m\right)$ that gives a straight line are sought (Figure 2.8). 
Table 2.1. Parameters used for the base case

\begin{tabular}{|c|c|c|}
\hline \multicolumn{3}{|c|}{ Reservoir properties } \\
\hline Temperature $^{(\mathrm{a})}$ & 745 & ${ }^{\circ} \mathrm{R}$ \\
\hline Initial pore pressure, $p^{(\mathrm{a})}$ & 8,350 & psia \\
\hline Pore compressibility, $c_{p p}$ & $3.00 \times 10^{-6}$ & $1 / \mathrm{psi}$ \\
\hline Porosity, $\phi^{(\mathrm{a})}$ & 0.06 & - \\
\hline \multicolumn{3}{|l|}{ Sorption properties } \\
\hline Grain density, $\rho_{\text {grain }}$ & 166 & $\mathrm{lbm} / \mathrm{cft}$ \\
\hline Bulk density, $\rho_{\text {bulk }}{ }^{(\mathrm{b})}$ & 156 & $\mathrm{lbm} / \mathrm{cft}$ \\
\hline $\begin{array}{l}\text { Total organic grain volume per total grain volume, } \\
\qquad \varepsilon_{k S}\end{array}$ & 0.01 & - \\
\hline Langmuir volume, $V_{s L}$ & 100 & scf/short-ton \\
\hline Langmuir pressure, $p_{L}$ & 2,000 & psia \\
\hline \multicolumn{3}{|c|}{ Gangi's model parameters } \\
\hline Permeability at zero effective stress, $k_{o}$ & $100 \times 10^{-6}$ & $\mathrm{md}$ \\
\hline$m$ & 0.5 & - \\
\hline$p_{1}{ }^{(\mathrm{c})}$ & 26,000 & psia \\
\hline Confining pressure, $p_{c}{ }^{(c)}$ & 15,000 & psia \\
\hline Effective stress coefficient, $\alpha$ & 0.5 & - \\
\hline \multicolumn{3}{|l|}{ Gas properties } \\
\hline Composition & Methane $100 \%$ & \\
\hline Molecular weight, $M$ & 16 & lbm/lb-mol \\
\hline Specific gravity & 0.55 & - \\
\hline Free gas density at standard condition, $\rho_{s c, g a s}$ & 0.04 & $\mathrm{lbm} / \mathrm{cft}$ \\
\hline Pore diffusion coefficient, $D^{(\mathrm{d})}$ & $10^{-9}$ & $\mathrm{~m}^{2} / \mathrm{s}$ \\
\hline Diffusion coefficient for sorbed phase, $D_{S}^{(\mathrm{d})}$ & $10^{-9}$ & $\mathrm{~m}^{2} / \mathrm{s}$ \\
\hline
\end{tabular}

The values shown in the table are mainly collected from (a) Meyer et al. (2010), (b) Ambrose et al. (2012), (c) Heller and Zoback (2013), Heller (2013), and (d) Akkutlu and Fathi (2012) 
In Table 2.1, $p_{1}$ is based on the experimental data, however, $k_{o}$ is selected according to the typical values we see for shales, and $m$ is chosen at a mid-point between 0 and 1 . Confining pressure is estimated by calculating overburden pressure using a typical value for rock bulk density as well as the depth of the formation shown in Heller and Zoback (2013).

$$
\ln \left[1-\left(\frac{k_{g a s}-\mu D c_{g}}{k_{o}}\right)^{1 / 3}\right]=m \ln \left(p_{c}-\alpha p\right)-m \ln p_{1}
$$

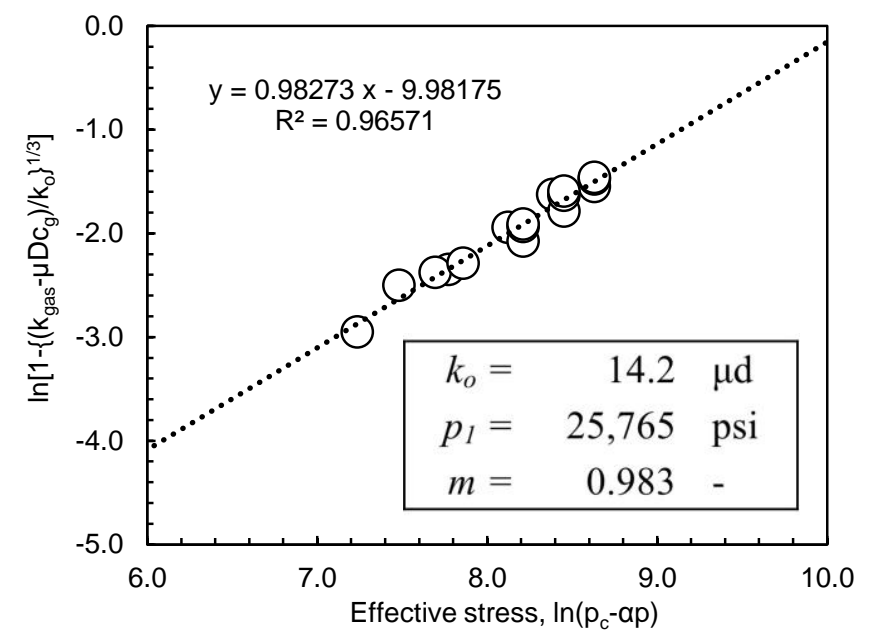

Figure 2.8. Experimental data matched with Gangi's model 
As for the gas properties, z-factor, coefficient of isothermal gas compressibility and viscosity are assumed to be pressure-dependent properties. The correlation proposed by Dranchuk and Abou-Kassem is used for z-factor, and Mattar correlation is used for the gas compressibility. Lee-Gonzalez-Eakin correlation is used for the gas viscosity. Kang et al. (2011) and Akkutlu and Fathi (2012) have estimated both pore diffusion coefficient, $D$, and diffusion coefficient for sorbed phase, $D_{\text {s. }}$ According to their investigation, the possible value of $D$ is approximately $10^{-9}\left[\mathrm{~m}^{2} / \mathrm{s}\right]$, and $D_{s}$ is between $10^{-8}-10^{-10}\left[\mathrm{~m}^{2} / \mathrm{s}\right]$. For this example, we selected $10^{-9}\left[\mathrm{~m}^{2} / \mathrm{s}\right]$ for both diffusion coefficients. Although it is likely that these diffusion coefficients change with the reservoir pressure, Bae and Do (2003), we assume these values are constant over pressure.

Figure 2.9 shows the four cases described in Table 2.2. Note that $k_{\text {init }}$ indicates permeability at initial reservoir pressure, which is calculated using Equation (2.1) with the value of the initial reservoir pressure given in Table 2.1. Firstly, let us compare the predicted apparent permeability for Case 1 and the Case 4 in Table 2.2 and Figure 2.9(a). The apparent permeability for Case 1 is decreasing with the decreasing pore pressure which can be confirmed from Equation (2.1). Decrease in pore pressure results in increase in effective stress according to its definition, i.e., $p_{c}-\alpha \cdot p$. The aperture of slit-shaped pores becomes smaller with the increasing effective stress, which results in decrease in permeability for Case 1. The effective stress builds up linearly, hence, the permeability decreases monotonically as the pore pressure is reduced. No molecular transport mechanism comes into play in the case of liquid. The predicted apparent permeability for gas, i.e., the Case 4, on the other hand, shows a trend similar to that with liquid at high 
pressure. The molecular transport comes in as a positive effect on permeability as the pore pressure is lowered further. Consequently, the permeability reduction rate decreases, reaching a zero rate (a minimum permeability) at around 4,000 psia for the base-case values listed in Table 2.1. Interestingly, below 3,000 psia, the predicted permeability improves rather dramatically. This increase in permeability indicates that the overall transport is improved even though the inorganic matrix pores are closed. The permeability increase in this case is purely driven by the molecular transport mechanisms, i.e., the diffusion of free and adsorbed molecules. This results in relatively large difference (nonDarcian effects) compared to $k_{\text {liquid }}$.

Table 2.2. Cases description for quantitative analysis of shale permeability

\begin{tabular}{|c|c|c|}
\hline $\begin{array}{c}\text { Case } \\
\text { No. }\end{array}$ & Apparent permeability model & Description \\
\hline 1 & $k_{\text {liquid }}=k_{m}$ & $\begin{array}{c}\text { Only the liquid transport } \\
\text { included }\end{array}$ \\
\hline 2 & $k_{g a s}=k_{m}+\mu D c_{g}$ & $\begin{array}{c}\text { Only the free gas phase } \\
\text { transport included }\end{array}$ \\
\hline 3 & $k_{g a s}=\mu D_{s} \frac{V_{s L} \rho_{g r a i n} B_{g}}{\varepsilon_{k s}} \frac{p_{L}}{\left(p+p_{L}\right)^{2}}$ & $\begin{array}{c}\text { Only the sorbed gas } \\
\text { phase transport included }\end{array}$ \\
\hline 4 & $k_{g a s}=k_{m}+\mu D c_{g}+\mu D_{s} \frac{V_{s L} \rho_{g r a i n} B_{g}}{\varepsilon_{k s}} \frac{p_{L}}{\left(p+p_{L}\right)^{2}}$ & $\begin{array}{c}\text { New apparent } \\
\text { permeability given by } \\
\text { Equation (2.6) }\end{array}$ \\
\hline
\end{tabular}


(a)

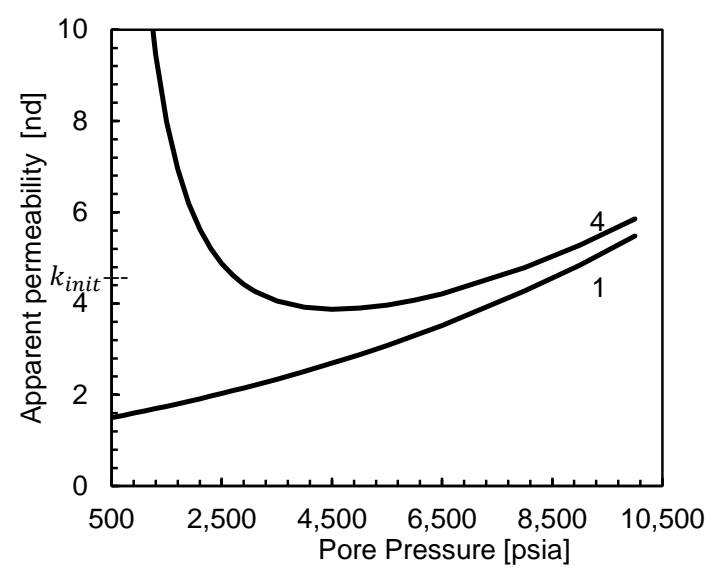

(c)

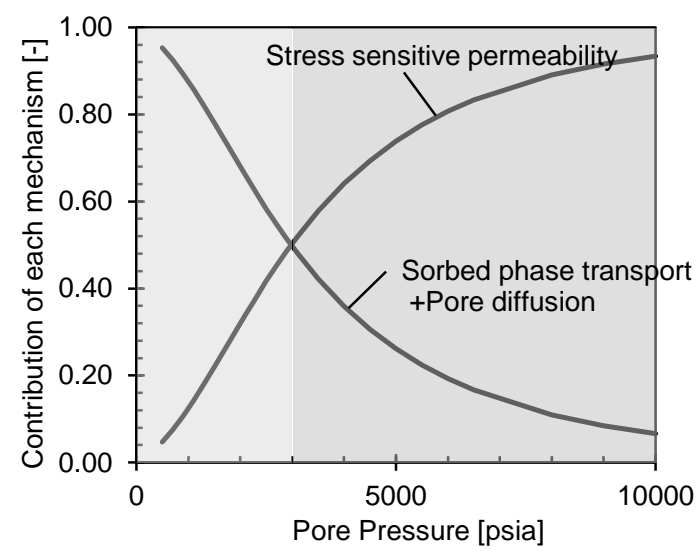

(b)

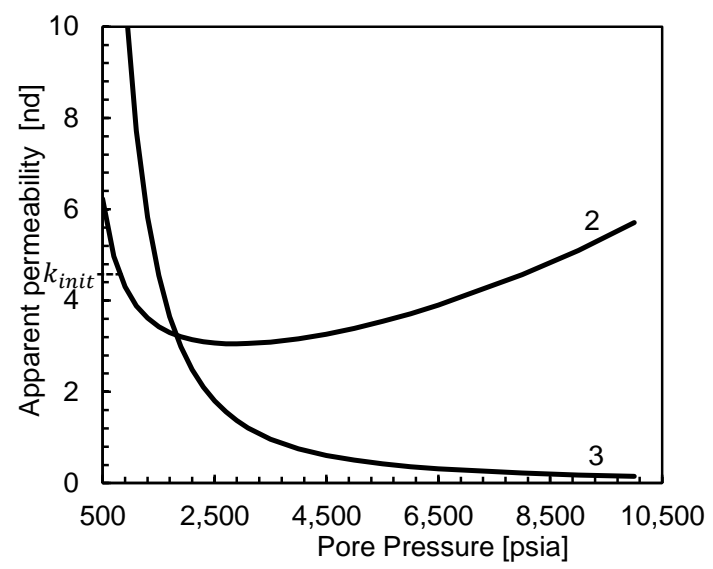

(d)

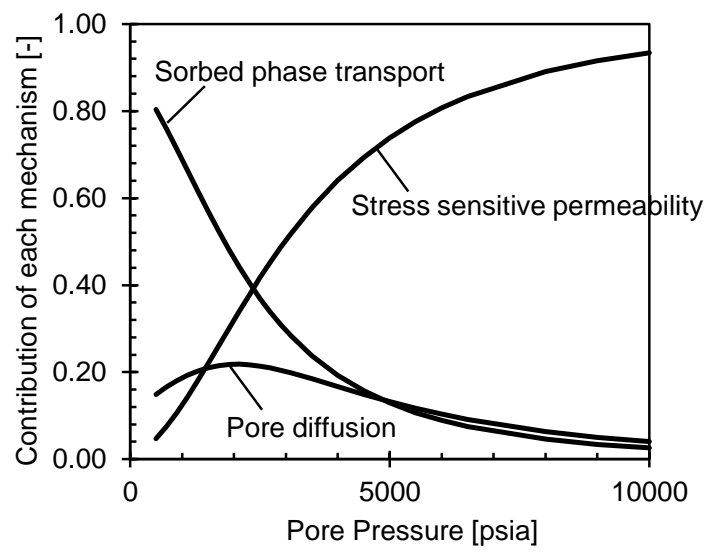

Figure 2.9. Analysis of the derived apparent permeability using the cases defined in Table 2.2 
(a)

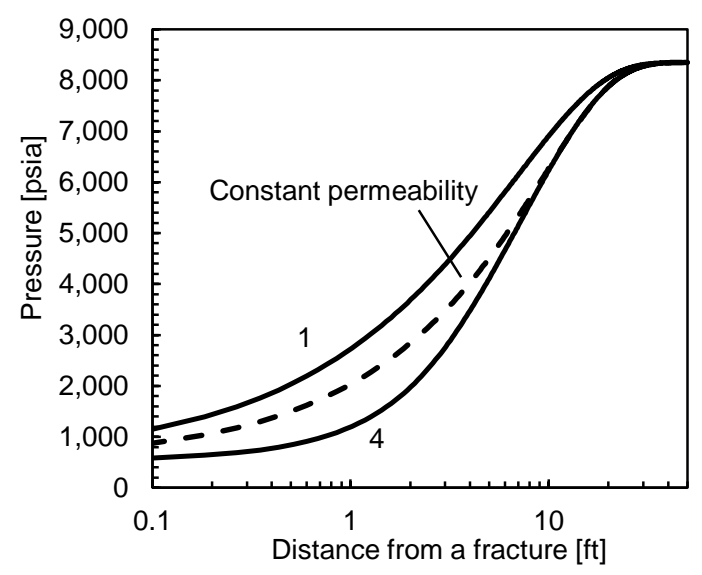

(b)

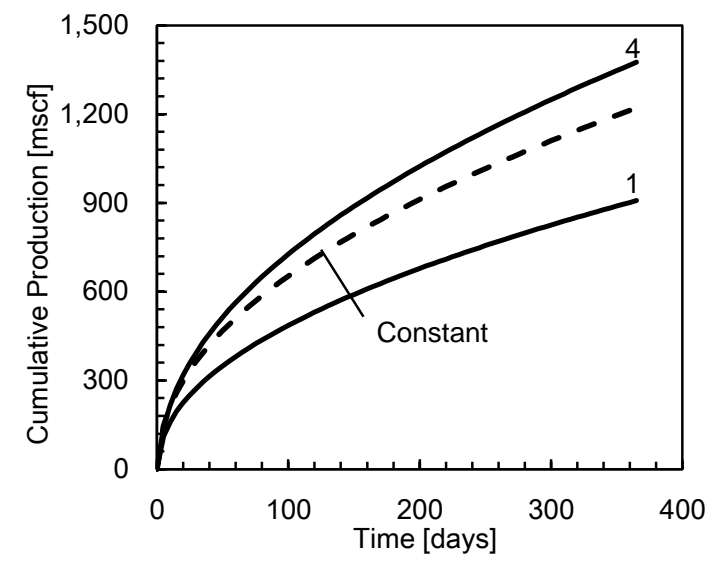

Figure 2.10. Influence of the apparent permeability model to production

Figure 2.9(b) shows the contribution of the molecular transport mechanisms on the apparent permeability for gas. Case 2 represents that particular case when the free gas phase transport is considered only. For this case, when the pore pressure is reduced and effective stress becomes larger, the aperture of slit-shaped inorganic pores becomes smaller. The subsequent reduction on flow is significant. We observe increase in permeability when the flux due to flow becomes comparable to flux due to pore diffusion of free gas molecules. At low pressures, indicated by light gray color in Figure 2.9(b), pore diffusion is the dominant mechanism. Case 3 in Figure 2.9(b) is the case when the apparent permeability includes only the contribution of the sorbed phase transport. In this case, the permeability improves as the pore pressure decreases. This is mainly because the formation volume factor, $B_{g}$, becomes larger as the pressure is reduced, consequently, the sorbed phase transport term, the third term on the right-hand-side of Equation (2.6), 
becomes larger. This trend seems to be consistent with some experimental observation previously reported by Bae and Do (2003). Our calculations using the base-case data shows that the sorbed phase transport becomes significant at pressures below 3,100 psia, shown in Figure 2.9(c) with lighter grey shade. The value of 3,100 psia is picked from Figure 2.9(c). The $y$-axis in Figure 2.9(c) and (d) shows the contribution of each flow mechanism shown in Equation (2.6). The first term is stress sensitive permeability, the second term is pore diffusion, and the third term is sorbed phase transport. The fraction of each term over the combination of the three terms is the $y$-axis shown in Figure 2.9(c) and (d). Going back to Figure 2.9(c), it shows the dominant mechanism switches from stress sensitive permeability to molecular transport effects around 3,100 psia. As for Figure 2.9(d), it shows that the major flow mechanism is stress sensitive permeability at higher pore pressure and sorbed phase transport emerges at lower pore pressure. Pore diffusion has a peak at the intermediate pore pressure but it does not become the most dominant flow mechanism.

In order to investigate the influence of the proposed apparent permeability on production, a 1D flow simulation is conducted. A bulk rock volume of $50 \mathrm{ft} \times 350 \mathrm{ft} \times 30$ $\mathrm{ft}$ (half interval of fractures, bi-wing fracture length, and fracture height respectively) with a planar fracture cell attached at the left side is considered. A sink (producer) is placed in the fracture cell so that the fluid flows from right to left. 1 year of production is simulated. As shown in Figure 2.10, three cases are prepared. Those are, Case 1 and 4 from Table 2.2 and constant permeability case (fixed at the initial value of Case 1, $k_{\text {init }}$ ). Figure 2.10(a) shows the pressure profiles after 1 year of production. Since the producer is placed in the 
fracture cell, pressures are lowest at the left and build up to the right. $x$-axis is shown in logarithmic scale and cannot show the pressure at the fracture. However, the sink term is controlled at bottom-hole pressure of 500 psia in all cases. Figure 2.10(b) shows cumulative production profiles for each case. Further detail on the simulation is in Section 2.4. Figure 2.10(b) shows the highest cumulative production for Case 4 and the lowest for Case 1. This is consistent with the observation made in Figure 2.9, since this figure shows higher permeability for Case 4 compared with Case 1 . Case 1 shows the lowest cumulative production because permeability decreases as pore pressure declines and effective stress increases. As a consequence, cumulative production is the lowest [Figure 2.10(b)], and pressure profile remains the highest among all [Figure 2.10(a)]. On the other hand, in Case 4, molecular diffusion and sorbed phase transport emerges as pore pressure decreases and apparent permeability doesn't decreases as low as Case 1, moreover, apparent permeability can increase at lower pore pressure [Figure 2.9(a)]. Then the cumulative production for Case 4 becomes the best as shown in Figure 2.10(b) and pressure profile shows the lowest pressure distribution in Figure 2.10(a).

\subsubsection{Sensitivity of Additional Parameters to the New Apparent Permeability}

In Equation (2.6), we identified eight parameters related to the stress-dependency, and molecular transport. These parameters are explicitly shown in Table 2.3. In order to investigate the impact of each parameter, we perturbed them one by one and observed the sensitivity. In this section the sample data shown in Table 2.1 is still used, hence Case 4 in Figure 2.9 is now the base case of our sensitivity analysis. We perturbed each parameter 
by $\pm 50 \%$, while making sure that the value used is still within a reasonable range. Sensitivity is quantified as the deviation of apparent permeability as is shown in Equation (2.20):

$$
\text { Deviation of } k_{\text {gas }}=\frac{k_{\text {gas }}-k_{\text {gas }, \text { base }}}{k_{\text {gas }, \text { base }}}
$$

Figure 2.11 through Figure 2.13 are the results. Figure 2.11 shows the sensitivity to the parameters associated with the free gas transport. Figure 2.12 shows the sensitivity of diffusion coefficient for sorbed phase, $D_{s}$, and fractional volume of kerogen grain, $\varepsilon_{k s}$. Figure 2.13 shows the sensitivity due to Langmuir parameters $V_{s L}$ and $p_{L}$. Clearly, the apparent permeability is the most influenced by Gangi's stress-dependency parameters, $m, p_{1}$, and to a somewhat less extent, $\alpha$. According to the original paper, Gangi (1978) assumes that the surface of a slit shaped flow path is rough and can be modelled as a bed of nails. In the model, $m$ represents a distribution of the height of nails. If the heights are close to uniform with a few shorter nails, then $m$ becomes close to unity. If there are a variety of heights, then $\mathrm{m}$ becomes close to zero. Figure 2.11(a) shows a trend that the stress-dependent permeability declines faster for smaller $m$. This is because the aperture of the slit becomes more sensitive to effective stress change. Imagine a bed of nails with a variety of heights, it is easier to close the slit if there are fewer nails that touch both surfaces. Larger $m$ corresponds to almost uniform heights of nails. This makes more difficult to close the slit because there are more nails supporting the slit from closure. As the pressure decreases the apparent permeability curves follow their own corresponding paths according to the $\mathrm{m}$ values, and converge to zero at low pressure, where viscous flow 
becomes negligible and molecular transports become the dominant transport mechanism.

Figure 2.11(b) shows the sensitivity of $p_{1}$. This parameter represents the required effective stress to close the slit-shaped pores completely. As $p_{1}$ becomes larger, it becomes more difficult to close the slit-shaped pores. Eventually, permeability reduction due to decreasing pore pressure (increasing effective stress) becomes less and less. Figure 2.11(c) shows the sensitivity of $\alpha$. This is an effective stress coefficient, which appears in stress sensitive term [the first term in Equation (2.6)]. The influence of $\alpha$ decreases with decreasing pore pressure in Figure 2.11(c). This is consistent with the observation in Figure 2.9(d). As pore pressure decreases, the stress sensitive term in Equation (2.6) becomes relatively smaller compared to the other molecular transport effects. This leads to the diminishing influence of $\alpha$ in Figure 2.11(c). The last plot in Figure 2.11, Figure 2.11(d), shows the sensitivity of $D$. This is a diffusion coefficient for pore diffusion, which is shown as the second term in Equation (2.6). From Figure 2.11(d), the variation of $D$ does not influence much on the apparent permeability regardless of the pore pressure. However, the figure shows a peak at a lower pressure. This is because of the relative magnitude of the pore diffusion term. As pore pressure decreases, the two molecular transport terms start picking up. However sorbed phase transport, the third term in Equation (2.6), increases more than pore diffusion, and that leads to limited influence coming from pore diffusion.

Figure 2.12 shows the sensitivity of parameters related to sorbed phase transport, $D_{s}$ and $\varepsilon_{k s}$. We observe a slight enhancement on apparent permeability for larger diffusion coefficient. On the other hand, if you look at Figure 2.12(b), kerogen grain volume 
fraction, $\varepsilon_{k s}$, works oppositely. This variable explicitly appears in Equation (2.6) as a reciprocal. Thus for smaller $\varepsilon_{k s}$, apparent permeability seems to increase.

Finally, Figure 2.13 shows the sensitivity of Langmuir parameters. Figure 2.13(a) shows the sensitivity of $V_{s L}$, and Figure 2.13(b) shows the sensitivity of $p_{L} . V_{s L}$ have intermediate impact on apparent permeability, whereas $p_{L}$ is one of the least influential parameters among all. The effect of $V_{s L}$ is also simple. If you consider Equation (2.6), this parameter appears in sorbed phase transport term, and it shows larger $V_{s L}$ results in larger sorbed phase transport. This is why the effect of changing $V_{s L}$ appears at lower pressure region.

Table 2.3. List of parameters for the sensitivity analysis

\begin{tabular}{|c|c|c|c|}
\hline \multicolumn{2}{|c|}{ Parameters } & Unit & Range \\
\hline \multirow{3}{*}{ Viscous flow term } & $m$ & - & $0.25 \sim 0.75$ \\
\cline { 2 - 4 } & $p_{1}{ }^{(\mathrm{a})}$ & $\mathrm{psi}$ & $13,000 \sim 39,000$ \\
\cline { 2 - 4 } & $\alpha$ & - & $0.25 \sim 0.75$ \\
\hline Pore diffusion & $D^{(\mathrm{b})}$ & $\mathrm{m}^{2} / \mathrm{s}$ & $0.5 \times 10^{-9} \sim 1.5 \times 10^{-9}$ \\
\hline Sorbed phase transport & $D_{s}{ }^{(\mathrm{b})}$ & $\mathrm{m}^{2} / \mathrm{s}$ & $0.5 \times 10^{-9} \sim 1.5 \times 10^{-9}$ \\
\hline Kerogen volume & $\varepsilon_{k s}$ & - & $0.005 \sim 0.015$ \\
\hline Langmuir parameters & $V_{s L}{ }^{(\mathrm{c})}$ & scf/ton & $50 \sim 150$ \\
\cline { 2 - 4 } & $p_{L}{ }^{(\mathrm{c})}$ & psia & $1,000 \sim 3,000$ \\
\hline
\end{tabular}

(a) Heller and Zoback (2013), Heller (2013), (b) Akkutlu and Fathi (2012), (c) Santos and Akkutlu (2013) 
(a)

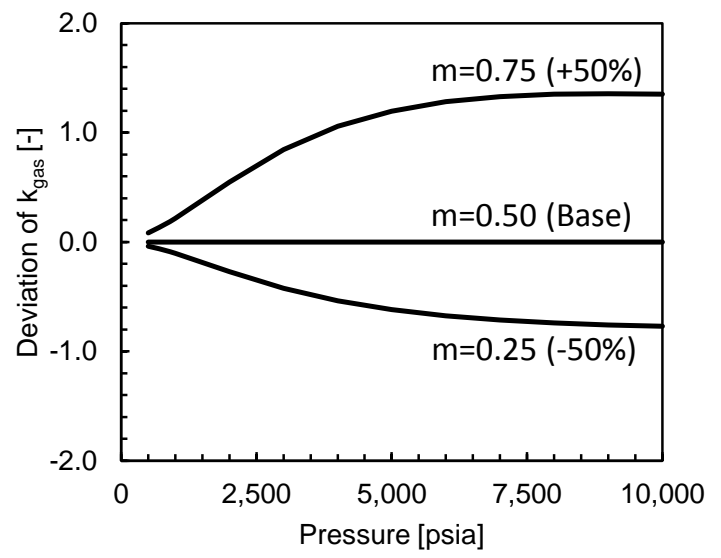

(c)

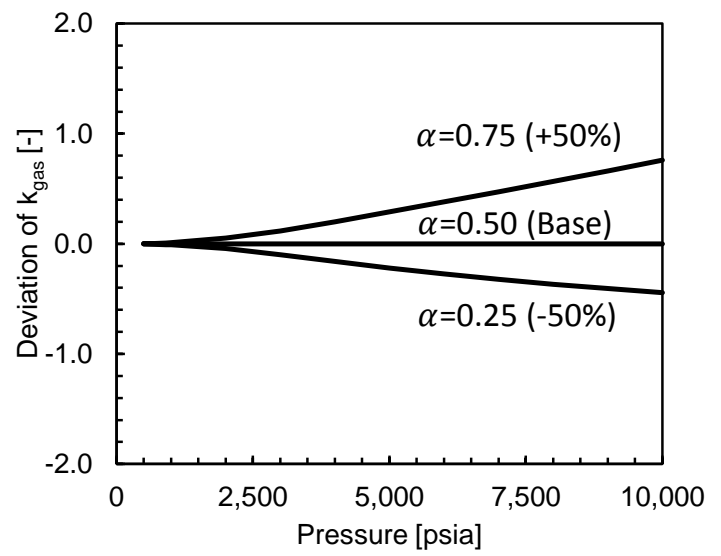

(b)

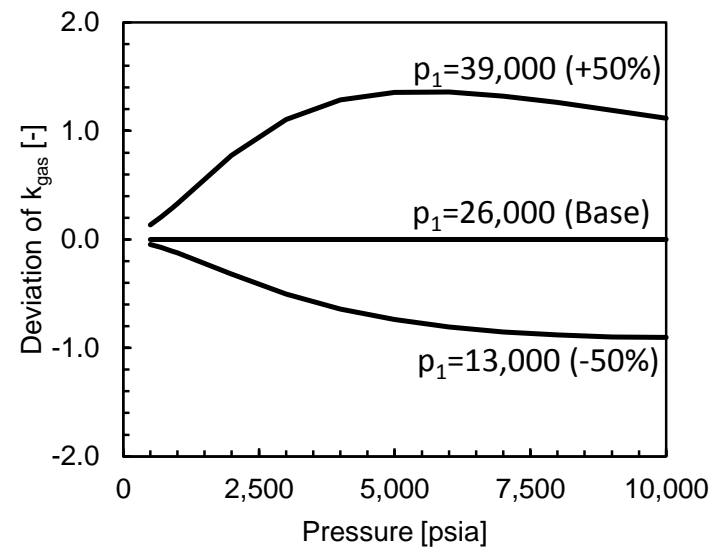

(d)

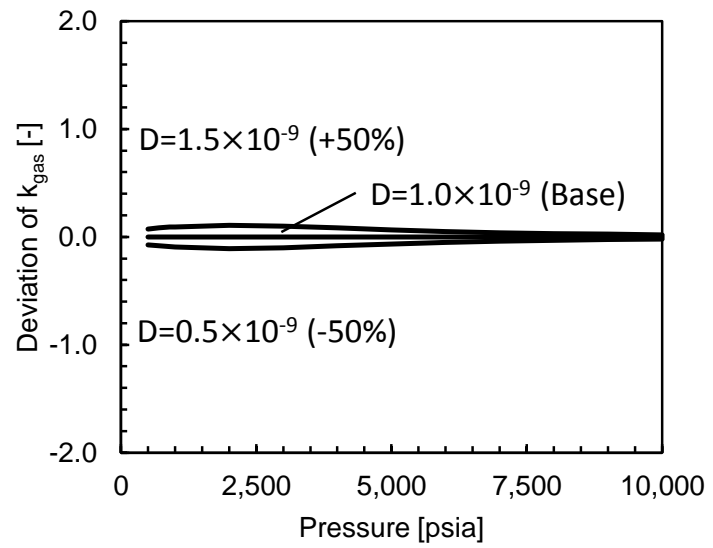

Figure 2.11. Sensitivity of Gangi's model parameters (a) $m$, (b) $p_{1}$, (c) $\alpha$, (d) $D$ 
(a)

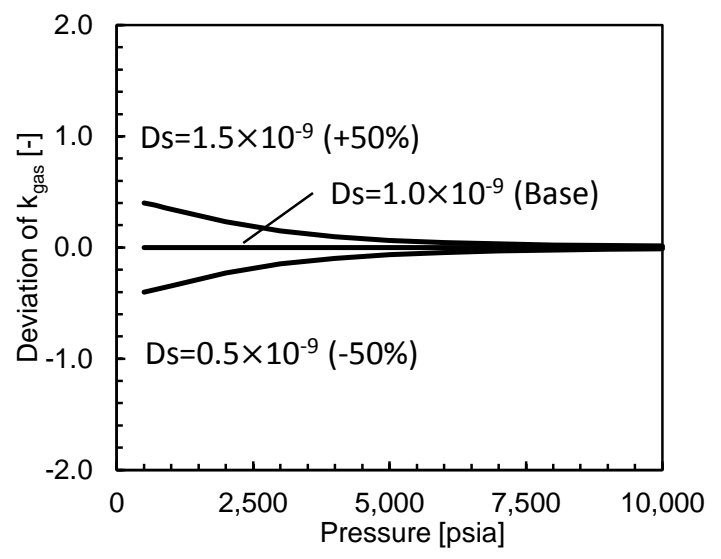

(b)

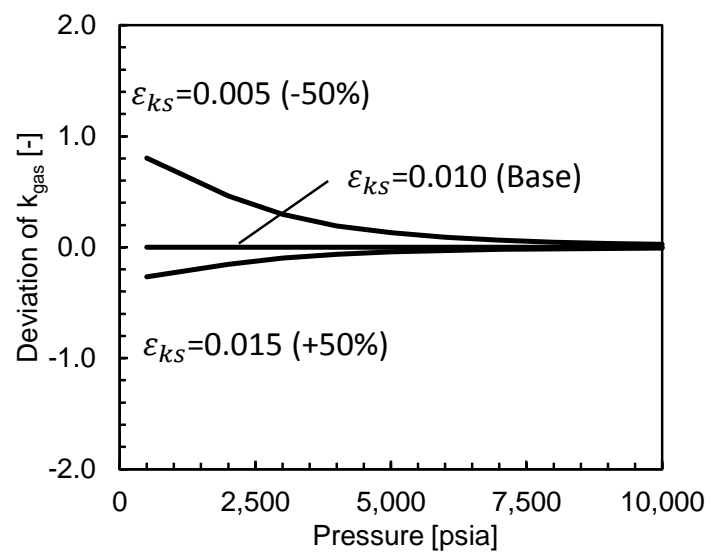

Figure 2.12. Sensitivity of the sorbed phase (a) $D_{s}$, (b) $\varepsilon_{k s}$

(a)

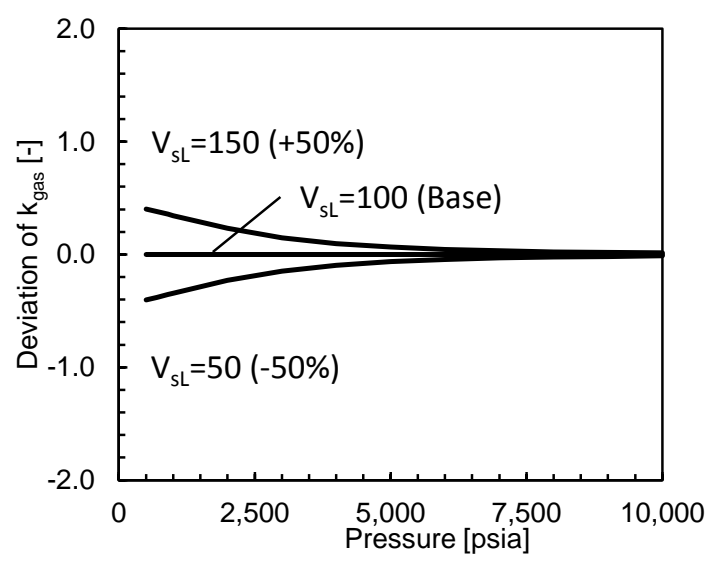

(b)

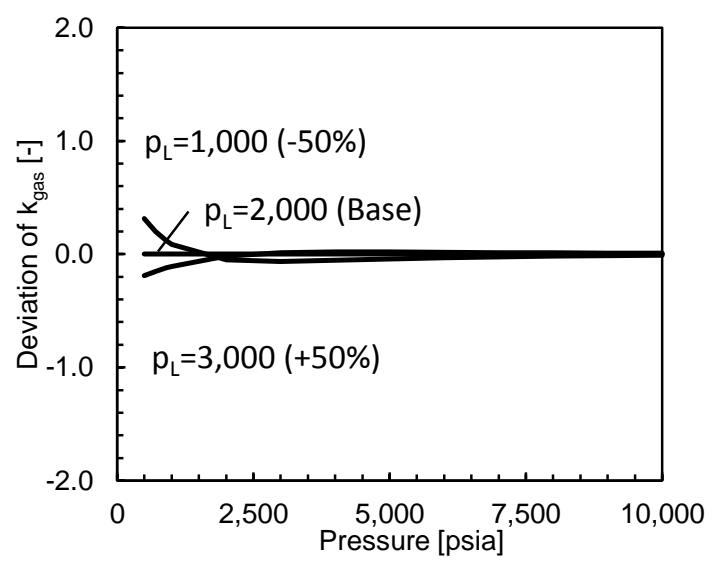

Figure 2.13. Sensitivity of Langmuir parameters (a) $V_{s L}$, (b) $p_{L}$ 


\subsection{Description on the In-house Flow Simulator}

\subsubsection{Configuration of a Sector Model and the Description of the Flow Simulator}

A simple $1 \mathrm{D} / 2 \mathrm{D}$ single phase flow simulator is developed assuming the fluid is gas. This simulator assume the fluid as a single pseudo-component whose properties, such as viscosity, compressibility, formation volume factor, are calculated from correlations basically using gas specific gravity. For further description on the correlations, please refer to Section 2.3.2.

The configuration of a sector model is shown in Figure 2.14. Assuming a horizontal well with multi-stage hydraulic fractures, a smallest flow unit with a fracture and adjacent matrix is taken into consideration. Figure 2.14(a) shows the one for 2D case. One wing out of two is modeled. The fracture is expressed as a single cell in $x$ - and $z$ direction, and multiple cells in $y$-direction. A sink is placed at the left bottom cell, which is one of the fracture cells, to represent a wellbore. The sector model has only one cell in $z$-direction, whose thickness is equal to the hydraulic fracture height, and has multiple cells in $x$ - and $y$-directions. As for 1D case, the extent of the sector model is doubled in $y$ -

direction containing a bi-wing fracture at the left of the model [Figure 2.14(b)]. The sink is placed at the center of the fracture cell for 1D case. And the model is discretized in $x$ direction. 
(a) A Sector Model with a Single Fracture for 2D flow simulation

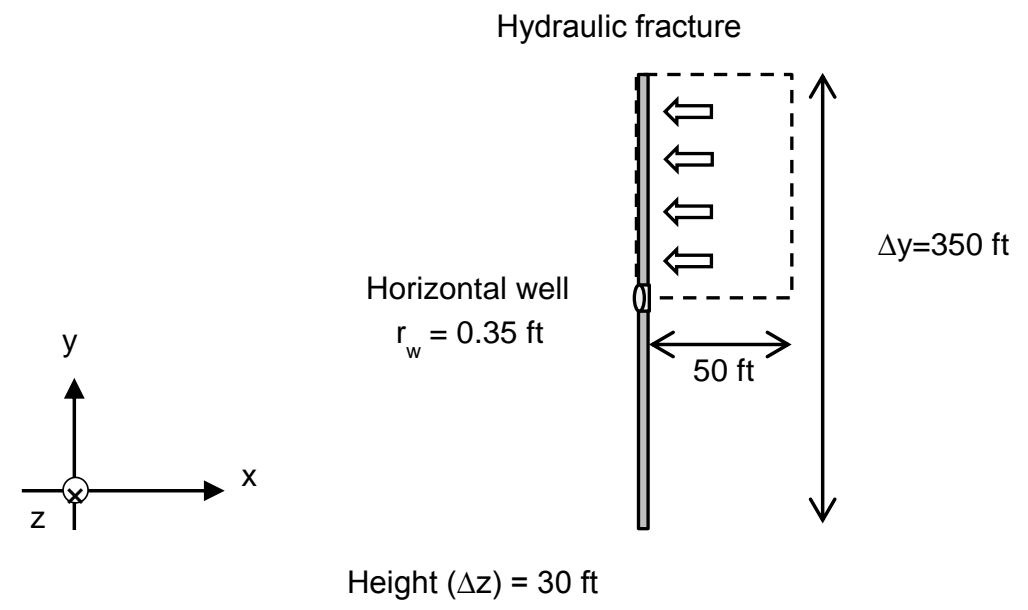

(b) A Sector Model with a Single Fracture for 1D flow simulation

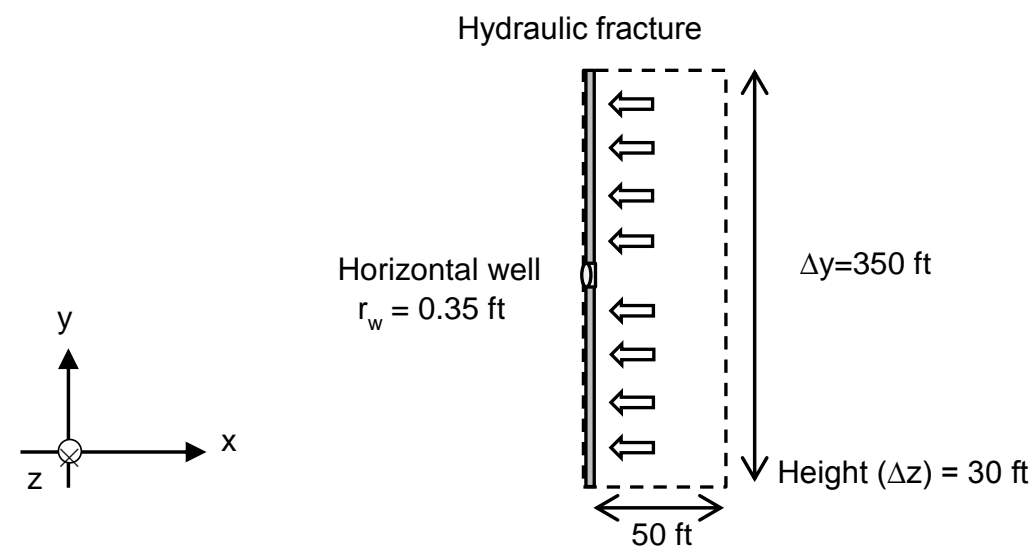

Figure 2.14. A Sector Model with a Single Fracture for 1D/2D flow simulation 
The flow simulation starts from discretizing the following mass balance equation for shale matrix, Equation (2.21). The unit of this equation is in mole per unit bulk volume, and we are going to multiply it with grid block volume later. In Equation (2.21), the left hand side shows an accumulation within the unit bulk rock volume and assumes sorption and free gas as a storage mechanism. $\phi$ is a connected total porosity and the volume reduction due to sorbed phase is not taken into account. When you turn to the right hand side, the first term shows net flux, where all the flow mechanisms are put together and shown as an apparent permeability, $k_{\text {gas }}$. A sink term per bulk rock volume is shown as $\tilde{q}$. As for fracture cells, the sorption term shown in the first term of the left hand side of Equation (2.16) will be gone, and the apparent permeability on the right hand side will become purely a term associated with convection.

$$
\frac{\partial}{\partial t}\left[\varepsilon_{k s}(1-\phi) C_{\mu}+\phi C\right]=\frac{\partial}{\partial x}\left(C \frac{k_{g a s}}{\mu} \frac{\partial p}{\partial x}\right)+\tilde{q}
$$

This equation is written in moles of gas per bulk volume of rock $(\Delta V)$. By reshaping the equation for a mass balance equation for a bulk rock volume and discretizing it in space and time, Equation (2.22) (2.24) can be derived. In this case, backward difference scheme is applied for discretization in time, and central difference scheme is applied for discretization in space. $i$ indicates a grid block number, and $n$ indicates time step. $A_{c}$ is an accumulation term, which is shown as Equation (2.23), and $T_{r}$ is a transmissibility term, which is shown in Equation (2.24). 


$$
\begin{aligned}
& A_{c_{i}}^{n+1}\left(p_{i}^{n+1}-p_{i}^{n}\right) \\
& =T_{r_{i+\frac{1}{2}}^{n+1}}^{n+1} p_{i+1}^{n+1}-\left(T_{r_{i+\frac{1}{2}}^{n+1}}^{n+} T_{r_{i-\frac{1}{2}}^{n+1}}^{n+1}\right) p_{i}^{n+1}+T_{r_{i-\frac{1}{2}}^{n+1}}^{n+1} p_{i-1}^{n+1}+q_{i}
\end{aligned}
$$

where,

$$
\begin{gathered}
A_{c_{i}}^{n+1}=\frac{\Delta V_{i}}{\Delta t}\left[C_{\mu} \varepsilon_{k s}\left\{\frac{(1-\phi) p_{L}}{\left(p+p_{L}\right) p}-\phi c_{p p}\right\}+C \phi c_{t}\right]_{i}^{n+1} . \\
T_{r_{i \pm \frac{1}{2}}^{n+1}}^{n}=\frac{\left(C A k_{g a s}\right)_{i \pm 1}^{n+1}\left(C A k_{g a s}\right)_{i}^{n+1}}{\left(C A k_{g a s}\right)_{i \pm 1}^{n+1}(\mu \Delta x)_{i}^{n+1}+(\mu \Delta x)_{i \pm 1}^{n+1}\left(C A k_{g a s}\right)_{i}^{n+1}}
\end{gathered}
$$

In Equation (2.24), the transmissibility is estimated at a block face as a harmonic average. $A$ is a cross section of a grid block assuming all the grid block shape of cuboid. A hydraulic fracture consists of one cell for 1D simulation whereas consists of 1174 cells for 2D simulation. In $x$-direction, there are 337 cells. The cell sizes in each flow direction is made small to match with the scale of a plug cores used in permeability measurement that we referred to. In total, there are 395,638 cells, which makes the problem large and increase the computation time.

Other inputs for rock properties and fluid properties are same as the ones described in Section 2.3.2. Additional information required here is some properties for fracture cells and grid block sizes. Porosity of the fracture cells are set to be $\phi_{f}=0.3$. The width of the fracture cell in $x$-direction is set as 0.4 inch. As for matrix cells, porosity is set to be 0.06 . The diffusion coefficients used in the simulation are based on Kang et al. (2011) and Akkutlu and Fathi (2012), and the values were estimated based on experiments using core 
plug under confining stress. We followed this and set the width of a matrix cell as 4.544 $\mathrm{cm}$.

As for the sink term, $q_{i}$, this is in moles per second and defined only for the fracture cell, and computed by Equation (2.25). We set it as a bottom-hole pressure control. Flowing bottom-hole pressure, $p_{w f}$, is set to be 500 psia. The well is modeled based on Peaceman's well model for horizontal well case [Chen and Zhang (2009)], assuming there is no anisotropy for proppant permeability, $k_{f} . r_{o}$ is the equivalent radius from Peaceman's well model. $h_{w}$, which is completion interval in Equation (2.25), is set to be the fracture width, i.e., 0.4 inch. Skin is set as zero. In 1D simulation, all of the wellbore is in the grid block and $\sigma=1$ is used. As for 2D case, only half is in the cell, therefore $\sigma=0.5$ is used.

$$
\begin{gathered}
q_{i}=-\sigma C \frac{2 \pi k_{f} h_{w}}{\mu \ln \left(r_{o} / r_{w}\right)}\left(p-p_{w f}\right) \\
r_{o}=0.14\left(\Delta z^{2}+\Delta y^{2}\right)^{0.5} \ldots .
\end{gathered}
$$

A matrix equation is constructed based on Equation (2.22), and new pressure for each grid block is estimated by Newton-Raphson method for each time step $(\Delta t=5 \sim 30$ days) until 1 year.

\subsubsection{Comparison with a Commercial Simulator for Verification}

In order to check the validity of the developed simulator, the in-house simulator is compared with Eclipse. The cases compared are, Case 1) with all the storage mechanisms with fixed matrix and fracture permeabilities. Case 2) with all the storage mechanisms with changing matrix permeability and static properties for the fracture. Using these two 
cases, cumulative production profiles, pressure distributions after a year of production, and grid block pressure profiles for the one with sink are compared.

In order to account for sorbed phase as storage, the effective porosity, $\phi_{\text {eff, }}$, shown in Equation (2.27) is introduced. This equation is derived from the left hand side of Equation (2.21). By introducing this effective porosity, it is still possible to use the conventional description of the mass balance equation, as if there is no sorption.

$$
\phi_{e f f}=\varepsilon_{k s}(1-\phi) \frac{C_{\mu}}{C}+\phi \ldots \ldots \ldots \ldots \ldots \ldots \ldots \ldots \ldots \ldots \ldots \ldots
$$

Since Eclipse allows us to give information on rock properties, such as porosity and permeability, as a function of pore pressure, this feature is used to solve the mass balance equation of interest. The porosity as a function of pore pressure is given according to Equation (2.27) above, and permeability is given following the apparent permeability constructed in the previous Section 2.2. A part of the actual input is shown in the following page, with ROCKTAB keyword. The first column is pore pressure, and the second column is Pore Volume Multiplier and the third is Transmissibility Multiplier. Pore Volume Multiplier corresponds to the ratio of $\phi_{\text {eff }}$ over $\phi_{\text {eff }}$ at initial pore pressure. Transmissibility Multiplier corresponds to the ratio of $k_{g a s}$ over $k_{g a s}$ at initial pore pressure. 
(a) Case 1: Fixed matrix permeability

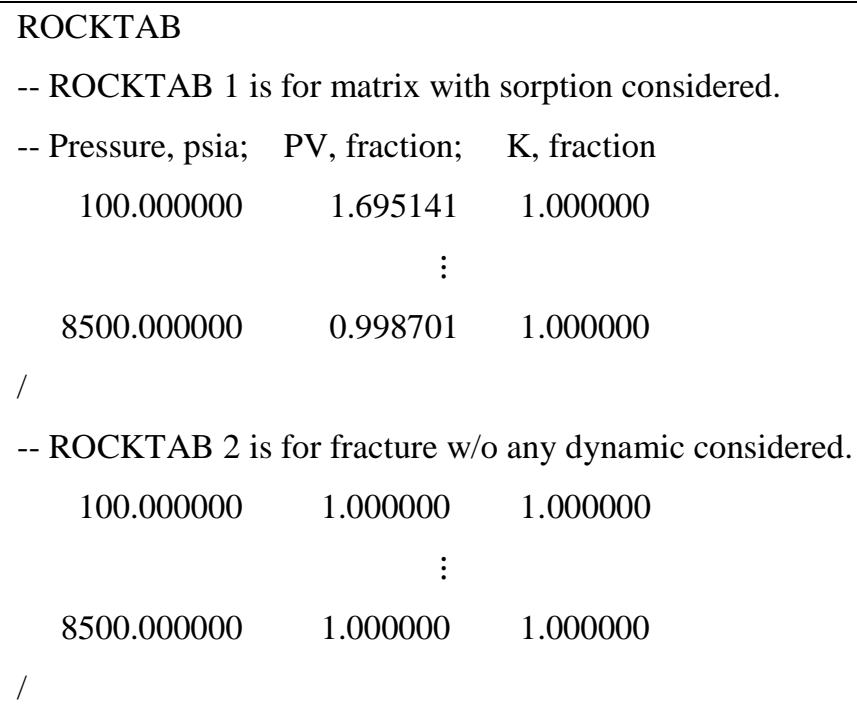

(b) Case 2: Dynamic matrix permeability with all the mechanisms

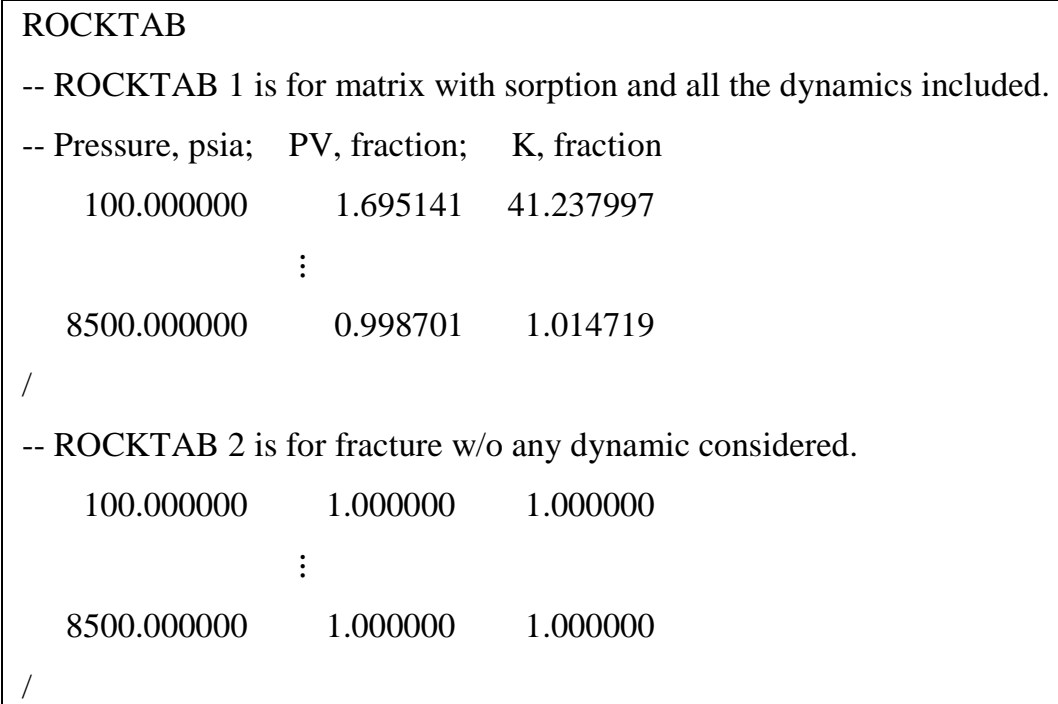

Figure 2.15. Sample ROCKTAB table for Eclipse input 
For the first case, the third column in ROCKTAB 1 is set at unity for all rows, and for the second case, it changes with pore pressure. ROCKTAB 2 is kept the same and both Pore Volume Multiplier and Transmissibility Multiplier are set as pressure independent parameters. In either case, Pore Volume Multiplier increases as pore pressure goes down. This trend acceptable only if coal bed methane option (COAL) is used. In addition, this option should be used along with dual porosity option (DUALPORO). However, the porosity model desired is a single porosity. To implement this, shape factor (SIGMA) of zero has to be used.

The results from each case run are shown in Figure 2.16 and Figure 2.17. Figure 2.16 is the results for Case 1 (fixed matrix permeability), and Figure 2.17 is for Case 2 (changing matrix permeability). For each figure, the following three items are compared. (a) Pressure distribution at day 360, (b) cumulative production, and (c) well block pressure profile are compared to show the validity of the in-house simulator. 
(a) Pressure distribution comparison @ day 360

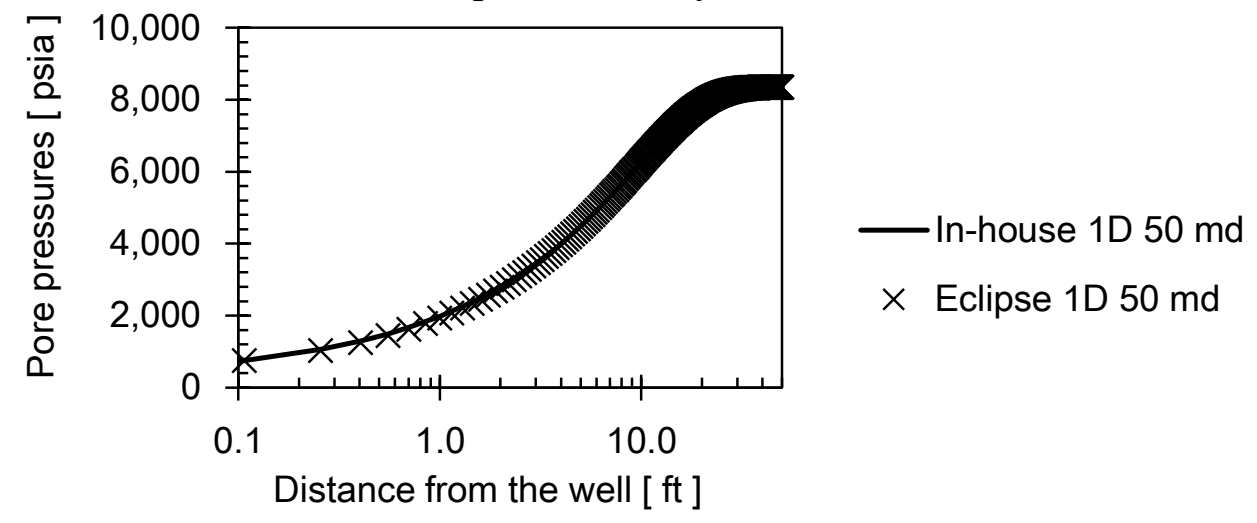

(b) Cumulative production comparison

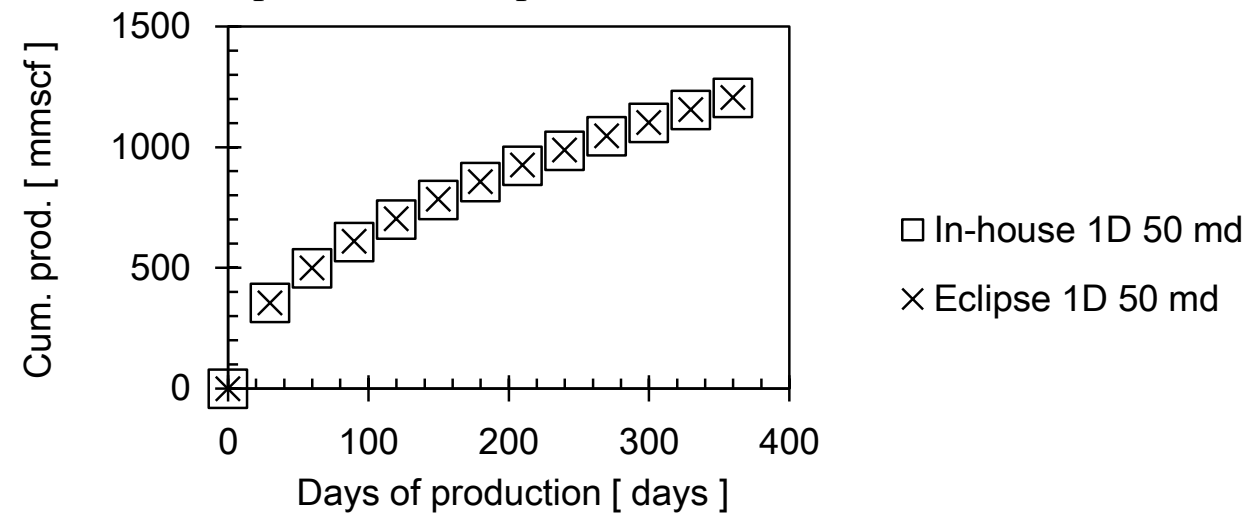

(c) Well Block Pressure (WBP) comparison

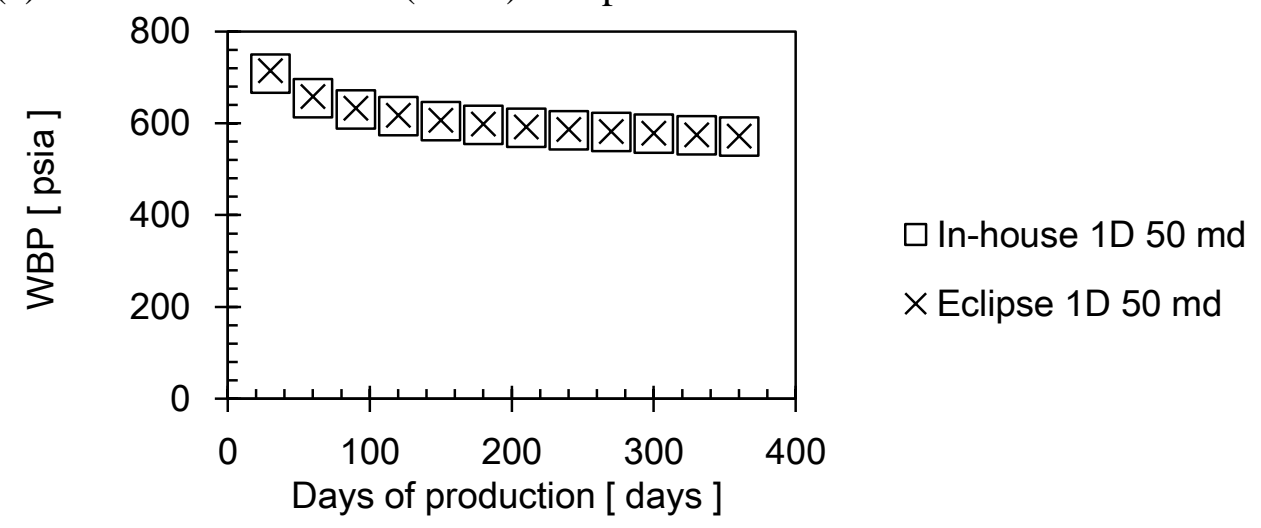

Figure 2.16. Comparison between Eclipse and the in-house simulator (Case 1) 
(a) Pressure distribution comparison@ day 360

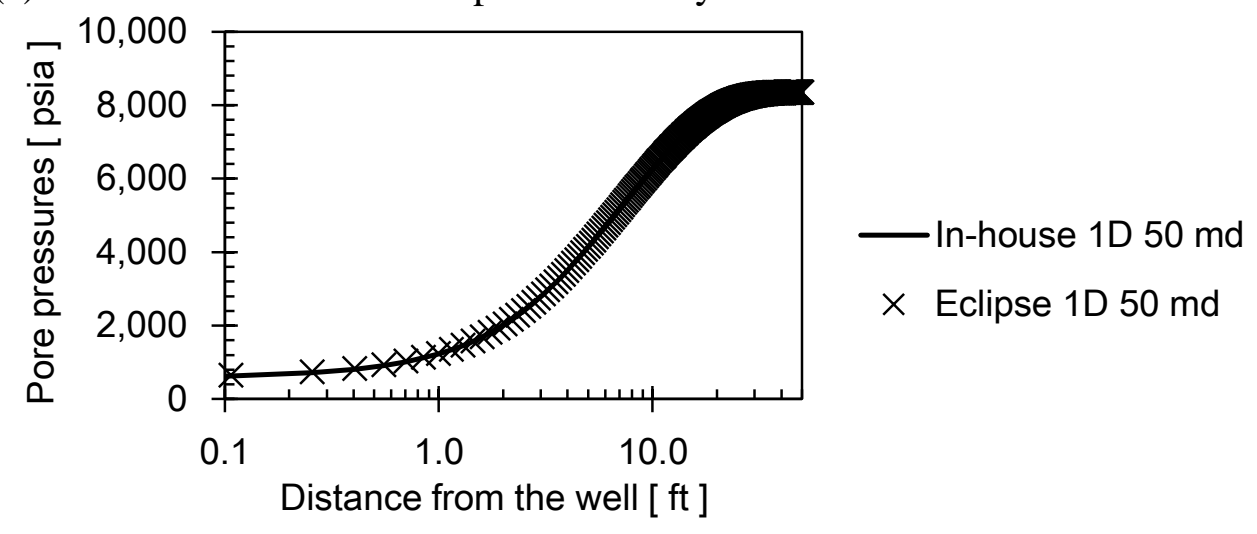

(b) Cumulative production comparison

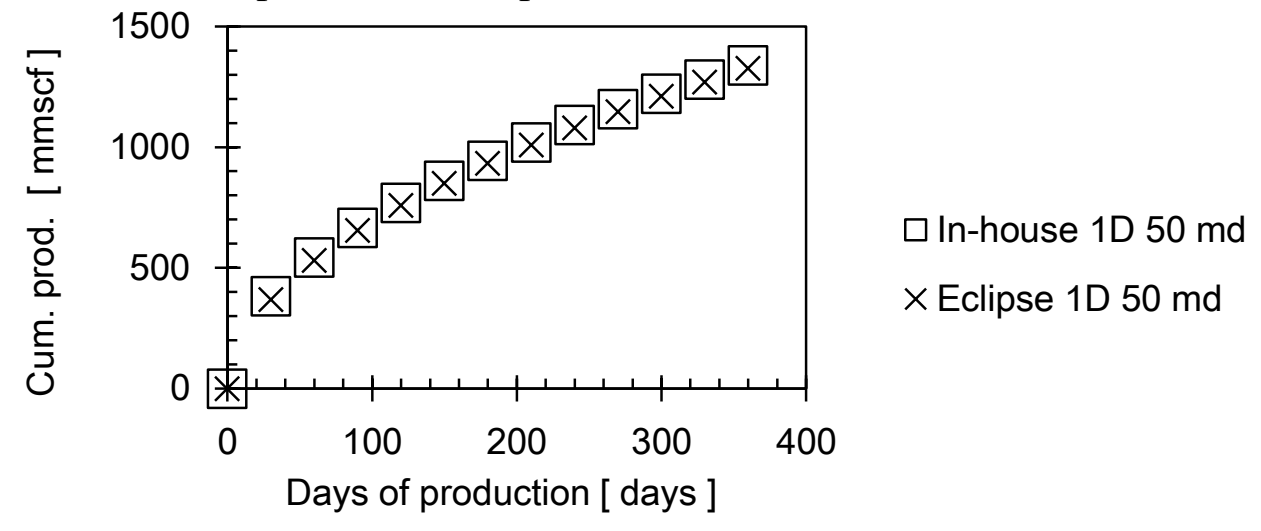

(c) Well Block Pressure (WBP) comparison

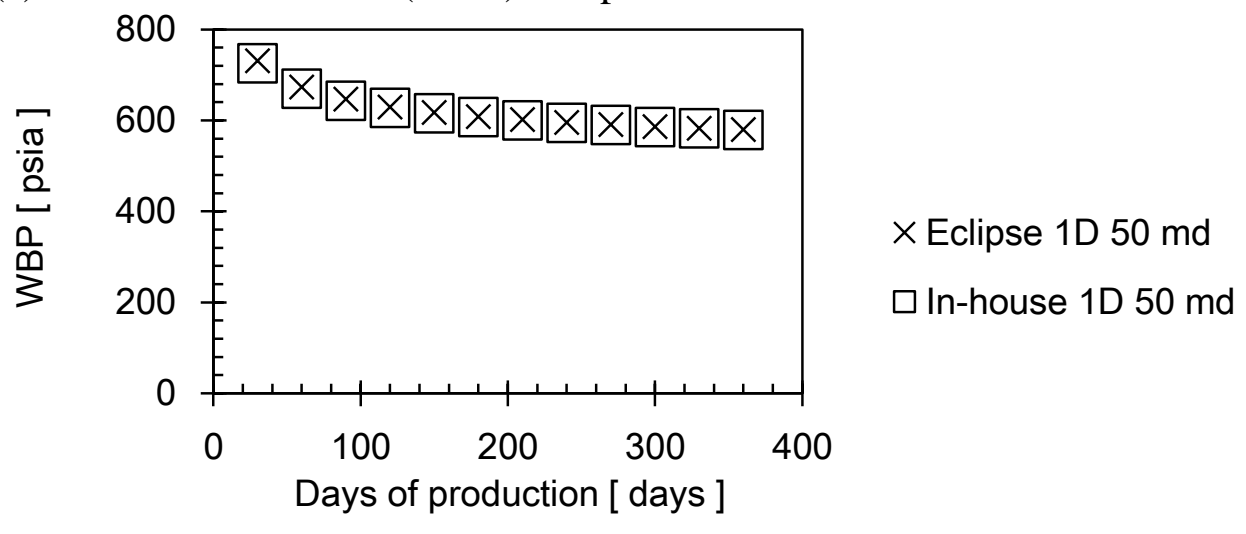

Figure 2.17. Comparison between Eclipse and the in-house simulator (Case 2) 


\subsection{Impact of the Dynamic Matrix Permeability on Production}

Complete form of the proposed apparent permeability for shale matrix is shown in Equation (2.28). In this section, fracture permeability is fixed at 50 md to look carefully at the effects coming from matrix. Then, eight parameters in the apparent permeability model are perturbed by $\pm 50 \%$ independently to check the effects on the cumulative gas production. The parameters are $\alpha, p_{l}, m, D, D_{s}, V_{s L}, \varepsilon_{k s}$, and $p_{L}$. The base case, and the range of perturbation is the same as the one shown in Section 2.3.3, Table 2.3.

$$
k_{\text {gas }}=k_{o}\left(1-\left(\frac{p_{c}-\alpha p}{p_{1}}\right)^{m}\right)^{3}+\mu D c_{g}+\mu D_{s} \frac{V_{s L} \rho_{g r a i n} B_{g}}{\varepsilon_{k s}} \frac{p_{L}}{\left(p+p_{L}\right)^{2}} \ldots
$$

Note that $V_{s L}$ is the only parameter among the eight parameters that influences in the accumulation term, i.e., the left hand side of the mass balance equation we are using. All the eight parameters control "how fast we can deplete the sector," but $V_{s L}$ can also affect the storage. Therefore, when this parameter is perturbed, it affects both deliverability and storage.

The result is shown in Figure 2.18 in terms of cumulative production (fraction). $y$-axis shows the list of parameters in Equation (2.28) used for this sensitivity analysis. $x$ axis shows the impact on cumulative production. Zero in the center indicates there is no difference from base case. For example, if you take a look at the first parameter on the list, $p_{1}$, red bar corresponds to the case when the parameter was perturbed by $+50 \%$, and this red bar shows around $37 \%$ increase in cumulative production compared to the base case. The blue bar shows the case when the parameter is perturbed by $-50 \%$, and the impact is about $28 \%$ decrease in cumulative production. 
Figure 2.18 shows that parameters shown in stress sensitive permeability model, the first term in Equation (2.28), is the most influential among all the parameters. Physical meaning of $p_{1}$ and $m$ are resistance to slit-shape pores closure. $p_{1}$ is the effective stress required to obtain zero permeability in Gangi's permeability model. Larger $p_{1}$ becomes, it requires more effective stress to close the slit. $m$ is related to the distribution of nail lengths in the same permeability model. Smaller $m$ represents rough surface of the slit with different nail lengths. Larger $m$ represents smoother surface with more uniform nail length distribution, which results in more resistance to fracture closure.

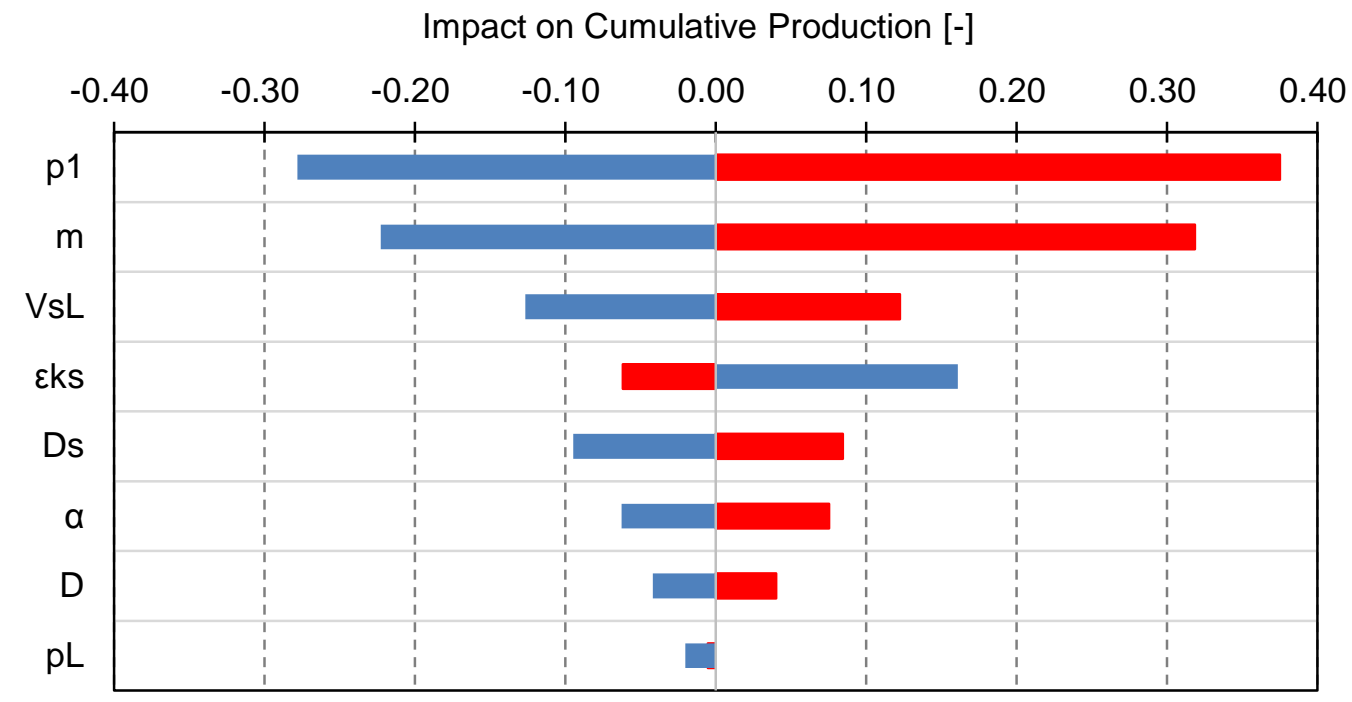

Figure 2.18. Sensitivity analysis of apparent permeability parameters on production $\left(\boldsymbol{k}_{f}=\mathbf{5 0} \mathrm{md}\right)$ 
Parameters in the sorbed phase transport, the third term in Equation (2.28), is the next influential term, and pore diffusion is the least influential term among the three mechanisms in the apparent permeability model.

Previously it was mentioned that $V_{s L}$ also affects the storage in the bulk rock. When $V_{s L}$ is increased by $50 \%$, this improves deliverability as well as fluid in place. On the other hand, the percentage of the fluid volume you can extract from the reservoir will decrease (see the right column in Table 2.4). In this table, the difference between the fluid volume under two different reservoir pressures are compared for three cases, i.e., high $V_{s L}$, low $V_{s L}$ and base cases. The high reservoir pressure of 8,350 psia is the initial reservoir pressure in this example, and 500 psia is the abandonment reservoir pressure. The right column shows the ratios of volumes, which are difference between initial and abandonment conditions divided by the initial fluid in place. With higher $V_{s L}$, the volume fraction on the right column decreases. This indicates the potential of getting less recovery factor for higher $V_{s L}$. This is something worth to keep in mind when one is to conduct a production forecast for a shale well.

Table 2.4. Influence of $V_{s L}$ on recovery factor

\begin{tabular}{|c|c|c|c|c|}
\hline & Fluid in $\mathrm{p}$ & mscf] & \multirow{3}{*}{$\begin{array}{c}\text { Fraction of the difference } \\
\text { of the fluid volume } \\
{[-]}\end{array}$} \\
\hline & & \multicolumn{2}{|c|}{ Reservoir pressure [psia] } & \\
\hline & & 8,350 & 500 & \\
\hline \multirow{3}{*}{$V_{S L}$} & low $(-50 \%)$ & 11,950 & 1,175 & 0.90 \\
\hline & base $(0 \%)$ & 13,603 & 1,586 & 0.88 \\
\hline & high $(+50 \%)$ & 15,256 & 1,996 & 0.87 \\
\hline
\end{tabular}


According to the observation in Figure 2.18, the biggest uncertainty in 1-year cumulative production comes from the stress sensitive permeability term, i.e., the first term in Equation (2.28). In order to see this more clearly, a simple numerical experiment is made. For each of the eight parameter in Equation (2.28), we have identified minimum, maximum, and mean value as shown in Table 2.3. According to these three values, assume a triangular distribution for each parameter. 500 sets of 8 parameters are drawn from these cumulative density function, and 500 runs are made to obtain one year cumulative production for each set. Table $\mathbf{2 . 5}$ shows the mean and variance of the 500 cumulative productions. Case 1 is when all the eight parameters are perturbed. Case 2 is when the parameters from the first term in Equation (2.28), the stress sensitive term, are perturbed. Case 3 is when the parameters in the third term, the sorbed phase transport term, are perturbed. And Case 4 is when the parameter in the second term, the pore diffusion term, is perturbed. The variance becomes small for Case 3 and 4 , which indicates the uncertainty mainly comes from the stress sensitive term.

Table 2.5. Effect on uncertainty coming from each flow mechanism in shale matrix $\left(k_{f}=50 \mathrm{md}\right)$

\begin{tabular}{|l|r|r|}
\hline & \multicolumn{1}{|c|}{$\begin{array}{c}\text { Mean } \\
(\mathrm{mmscf})\end{array}$} & $\begin{array}{c}\text { Variance } \\
\left(\mathrm{mmscf}^{2}\right)\end{array}$ \\
\hline Case 1 (all of the eight parameters perturbed) & 1.35 & $8.43 \times 10^{-2}$ \\
\hline Case 2 (only the stress sensitive term perturbed) & 1.39 & $7.25 \times 10^{-2}$ \\
\hline Case 3 (only the sorbed phase transport term perturbed) & 1.32 & $9.90 \times 10^{-3}$ \\
\hline Case 4 (only the pore diffusion term perturbed) & 1.35 & $4.68 \times 10^{-4}$ \\
\hline
\end{tabular}


Since the simulation is bottom-hole pressure controlled, it is possible to conduct production analysis such as the one shown by Wattenbarger et al. (1998). They derived an analytical production profile for a tight rock formation assuming a rectangular drainage with a planar fracture. According to their work, a half slope appears in early production, which indicates the infinite acting linear flow in the formation. Later on, the production profile deviates from the half slope and shows exponential behavior. This is the time when the flow starts feeling the extent of the reservoir.

This behavior is also captured by the in-house flow simulator, and the result is displayed in Figure 2.19. Dimensionless rate and time is calculated from Equation (2.29) and (2.30). Here, $k$ is matrix permeability, which a constant value is used for this case. $h$ is the reservoir thickness, $m(p)$ is pseudo-pressure, $q$ is the flow rate, and $T$ is the reservoir temperature. $t$ is production duration in days. $\phi$ is porosity and fixed value is used. Note that it is not the effective porosity in Equation (2.27) used for the calculation here. $\mu$ is gas viscosity, $c_{t}$ is total compressibility, $y_{e}$ is the distance from the hydraulic fracture to the end of the reservoir. Subscript $i$ for $\phi \mu c_{t}$ stands for the initial condition. Units are all in field unit for these equations.

$$
\begin{gathered}
\frac{1}{q_{D}}=\frac{k h\left[m\left(p_{i}\right)-m\left(p_{w f}\right)\right]}{1424 q T} \\
t_{D_{y e}}=\frac{0.00633 k t}{\left(\phi \mu c_{t}\right)_{i} y_{e}^{2}} \ldots \ldots
\end{gathered}
$$

Figure 2.19(a) shows the comparison between two cases with different storage mechanisms considered. The line in a black solid is the case when there is sorption as the 
additional storage mechanism. For this case, matrix and fracture permeability are fixed. The black dotted line, on the other hand, is the case without sorption. Whether sorption is taken into account or not, the shape of the production profile does not change much as Figure 2.19(a) shows. For either case, a straight line of a half slope first appears, followed by an exponential trend. This half slope is an indication of formation linear flow, and the exponential trend comes from the boundary effect of the closed reservoir. After the linear flow regime, the case without sorption declines a little faster than the other case.

Figure 2.19(b) shows the comparison between three cases with different transport mechanisms considered. The black solid line is the same case as the one shown in Figure 2.19(a). The black dashed line is when Gangi's model is used for matrix permeability, and the dash dot line shows the case when all the three transport mechanisms are considered. All the three lines does not show much difference for the linear flow regime, however it shows clear difference in the later flow regime. 
(a) The contribution of sorption as a storage mechanism

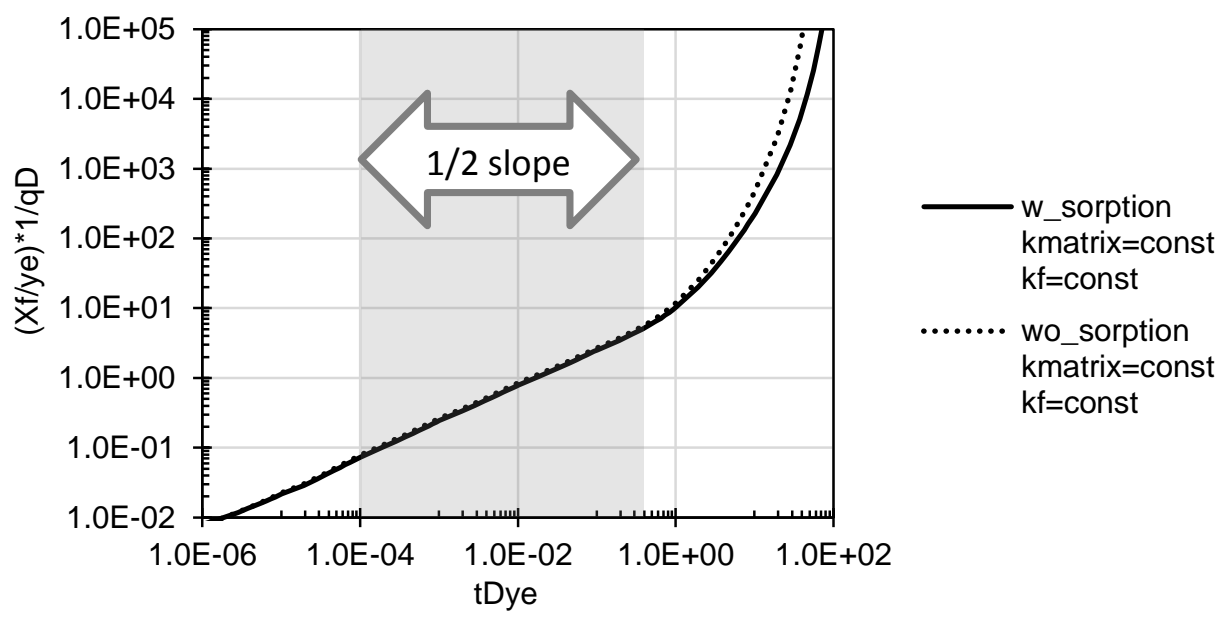

(b) The contribution of each flow mechanism

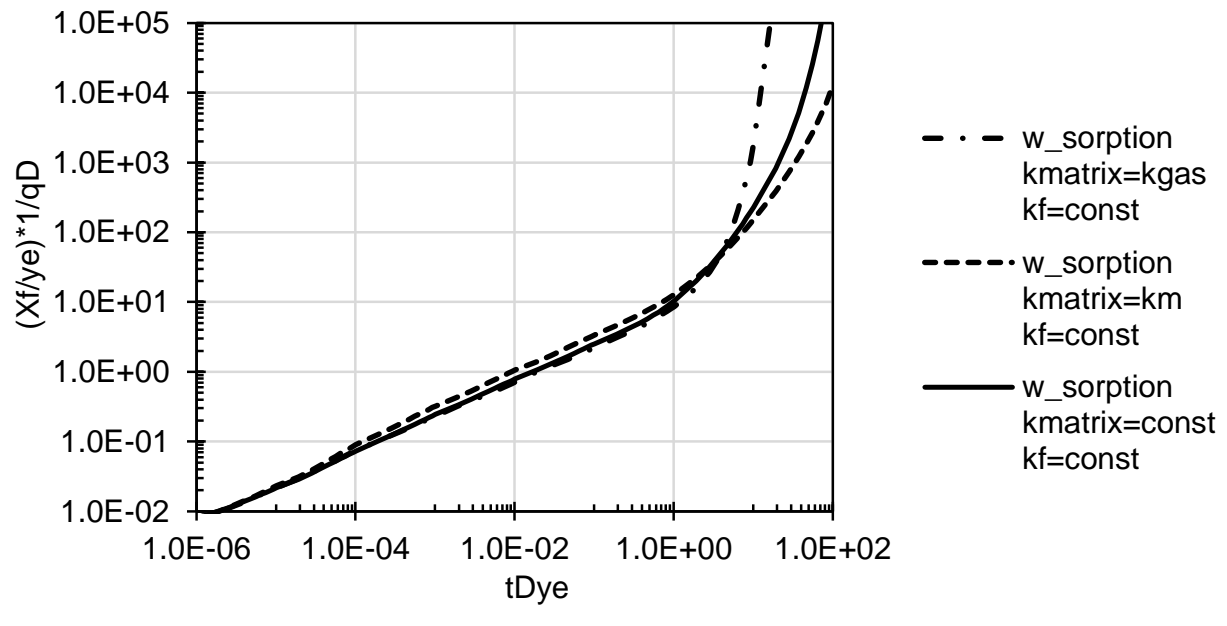

Figure 2.19. Production analysis on a shale reservoir featuring the dynamics in the matrix 


\subsection{Application of the Apparent Permeability Model to a Field Case}

Two sets of data from different formations in South America are used to show the application of the apparent permeability model. Here, the formations are named Formation 1 and 2. These formations have the following properties as shown in Table 2.6. According to this table, Formation 2 has higher $V_{s L}, \phi, \varepsilon k s$ and lower $p_{1}$ compared to Formation 1. Higher $V_{s L}$ and $\phi$ indicate that Formation 2 has more storage capacity. Suppose an abandonment pressure of 500 psia, then Formation 2 shows more recoverable (see Table 2.7). This storage capacity can lead to higher productivity. However, the resulting apparent permeability shown in Figure 2.20 indicates higher apparent permeability for Formation 1 compared to Formation 2. It is hard to tell which formation is more productive just by looking at the properties. Therefore, flow simulation is conducted for each formation and the resulting cumulative production is shown in Figure 2.21(b). The figure is consistent with the observation in Figure 2.20. The apparent permeability for Formation 1 is evaluated higher than that of Formation 2. Therefore, Formation 1 is more permeable and results in better production at the end of one year. Also, Figure 2.21(a) indicates that Formation 1 has larger drainage after one year of production due to the higher permeability. The reason for this behavior is the geomechanics. Despite the fact that Formation 2 has higher $\phi$ for free gas and more sorbed gas, it loses its productivity due to the lower $p_{1}$ value. Formation 1 has $27 \%$ more cumulative production compared to Formation 2 under the same bottom-hole pressure condition of 500 psia. Formation 2 has lower deliverability than Formation 1. Better deliverability can be achieved by lower bottom-hole pressure, longer fracture length, and more frequent fracture spacing. 
Table 2.6. Parameters used for the field case

\begin{tabular}{|c|c|c|c|}
\hline & Formation 1 & Formation 2 & \\
\hline \multicolumn{4}{|l|}{ Reservoir properties } \\
\hline Temperature & 665 & 680 & ${ }^{\circ} \mathrm{R}$ \\
\hline Initial pore pressure, $p$ & 6,948 & 7,114 & psia \\
\hline Pore compressibility, $c_{p p}$ & $1.00 \times 10^{-5}$ & $1.00 \times 10^{-5}$ & $1 / \mathrm{psi}$ \\
\hline Porosity, $\phi$ & 0.06 & 0.08 & - \\
\hline \multicolumn{4}{|l|}{ Sorption properties } \\
\hline Grain density, $\rho_{\text {grain }}$ & 156 & 143.5 & $\mathrm{lbm} / \mathrm{cft}$ \\
\hline Bulk density, $\rho_{\text {bulk }}$ & 143 & 143 & $1 \mathrm{bm} / \mathrm{cft}$ \\
\hline Total organic grain volume per total grain volume, $\varepsilon_{k s}$ & 0.057 & 0.152 & - \\
\hline Langmuir volume, $V_{S L}$ & 125 & 170 & scf/short-ton \\
\hline Langmuir pressure, $p_{L}$ & 1,523 & 1,000 & psia \\
\hline \multicolumn{4}{|l|}{ Gangi's model parameters } \\
\hline Permeability at zero effective stress, $k_{o}$ & $100 \times 10^{-6}$ & $100 \times 10^{-6}$ & md \\
\hline$m$ & 0.5 & 0.5 & - \\
\hline$p_{1}$ & 26,000 & 18,000 & psia \\
\hline Confining pressure, $p_{c}$ & 9,940 & 10,176 & psia \\
\hline Effective stress coefficient, $\alpha$ & 0.7 & 0.7 & - \\
\hline \multicolumn{4}{|l|}{ Gas properties } \\
\hline Composition & $100 \%$ Methane & $100 \%$ Methane & \\
\hline Molecular weight, $M$ & 16 & 16 & lbm/lb-mol \\
\hline Specific gravity & 0.55 & 0.55 & - \\
\hline Free gas density at standard condition, $\rho_{s c, \text { gas }}$ & 0.04 & 0.04 & $\mathrm{lbm} / \mathrm{cft}$ \\
\hline Pore diffusion coefficient, $D$ & $10^{-9}$ & $10^{-9}$ & $\mathrm{~m}^{2} / \mathrm{s}$ \\
\hline Diffusion coefficient for sorbed phase, $D_{S}$ & $10^{-9}$ & $10^{-9}$ & $\mathrm{~m}^{2} / \mathrm{s}$ \\
\hline \multicolumn{4}{|l|}{ Fracture geometry } \\
\hline Fracture width, $w_{f}$ & 0.1 & 0.1 & in \\
\hline Fracture half length, $X_{f}$ & 100 & 100 & $\mathrm{ft}$ \\
\hline Fracture height, $h_{f}$ & 50 & 50 & $\mathrm{ft}$ \\
\hline Fracture permeability, $k_{f}$ & 50,000 & 50,000 & md \\
\hline
\end{tabular}

Table 2.7. Petroleum in place for the given reservoir pressure conditions and recoverable (Unit: mmscf)

\begin{tabular}{|l|c|c|}
\hline & Formation 1 & Formation 2 \\
\hline PIIP @ Initial pressure & 14.1 & 18.6 \\
\hline PIP @ 500 psia & 2.0 & 3.0 \\
\hline Recoverable & 12.2 & 15.7 \\
\hline
\end{tabular}


(a) Formation 1

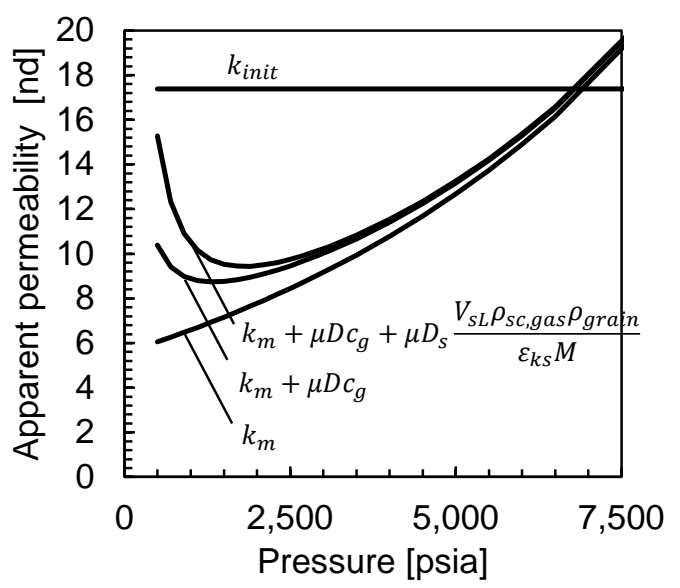

(b) Formation 2

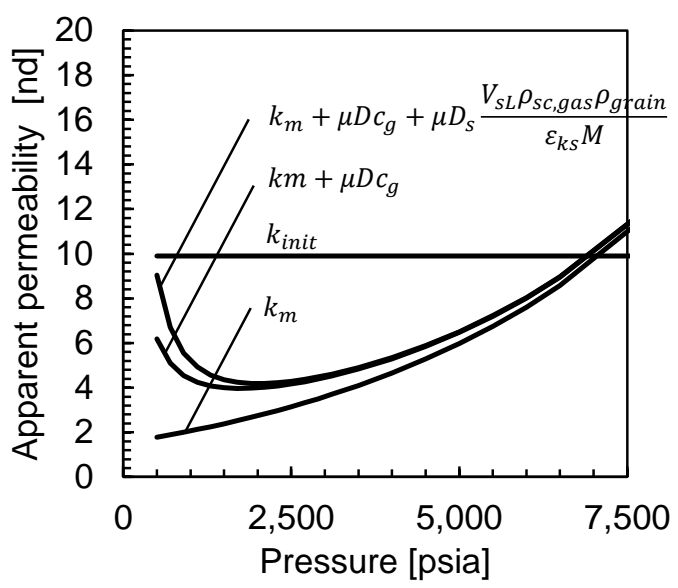

Figure 2.20. Apparent permeability for Formation 1 and Formation 2

(a) Pressure distributions @1 year

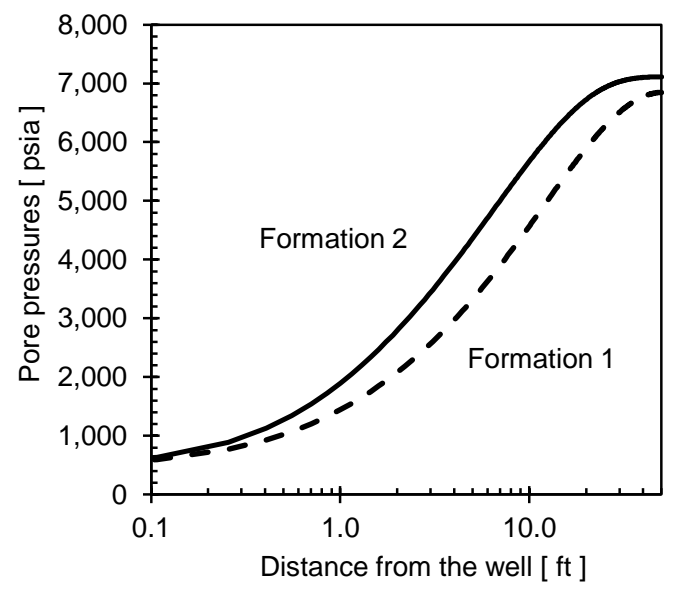

(b) Cumulative production profiles

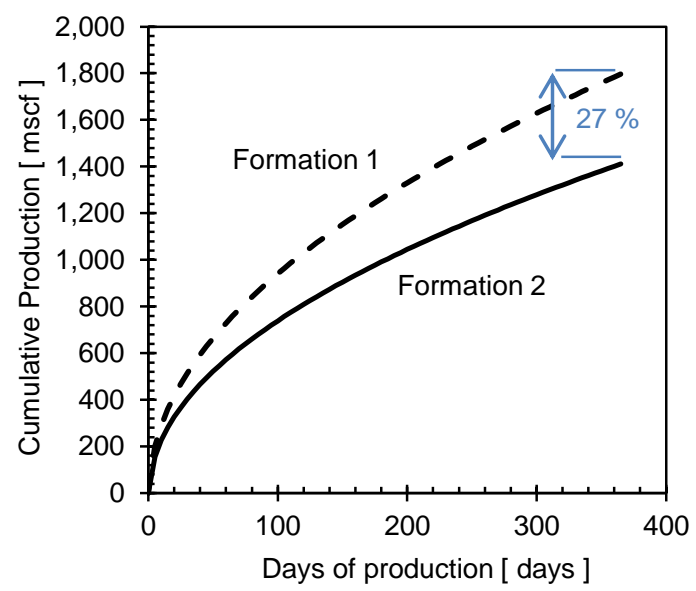

Figure 2.21. Pressure and cumulative production profiles for each formation 


\section{CONTRIBUTION OF FRACTURE FLOW TO PRODUCTION}

In the previous section, the flow mechanisms through shale matrix was the main focus, and the permeability in the hydraulic fracture was fixed at high value such that it could be considered as infinite conductivity. Here, in this section, the focus moves to the flow in the hydraulic fracture. The discussion begins with fixed fracture permeability with different values. First constant fracture permeabilities are analyzed as a base case before considering any mechanisms that adds more complexity. This would help us having an idea on what to expect when more complexity is added, such as elastic/inelastic stress sensitivity of the fracture permeability or non-Darcy effect. Then the discussion moves on to revisiting the flow mechanisms in the fracture including stress sensitive fracture permeability (elastic), time dependent fracture permeability, and the Forchheimer effect.

\subsection{Threshold Fracture Permeability}

Here, the fracture permeability is still constant, but 4 different values $(500 \mathrm{md}, 50$ $\mathrm{md}, 5 \mathrm{md}$, and $0.5 \mathrm{md}$ ) are investigated to see the effect on the production. Matrix permeability is set at approximately $4.5 \mathrm{nd}$. In this case fracture and matrix permeabilities are both insensitive to pressure. In order to investigate the effect of fracture permeability on production, a year of cumulative production profile and pressure distribution changing with the distance from the wellbore (along $x$-axis, $x=0$ indicates well bore location) were plotted. 
Figure 3.1 shows the results. As fracture permeability decreases, it will become difficult for the fracture cell to deliver the petroleum to the well. It seems the impact starts showing up around the permeability of $50 \mathrm{md}$ in either figures.

(a) Impact of fixed fracture permeability on pore pressure distribution

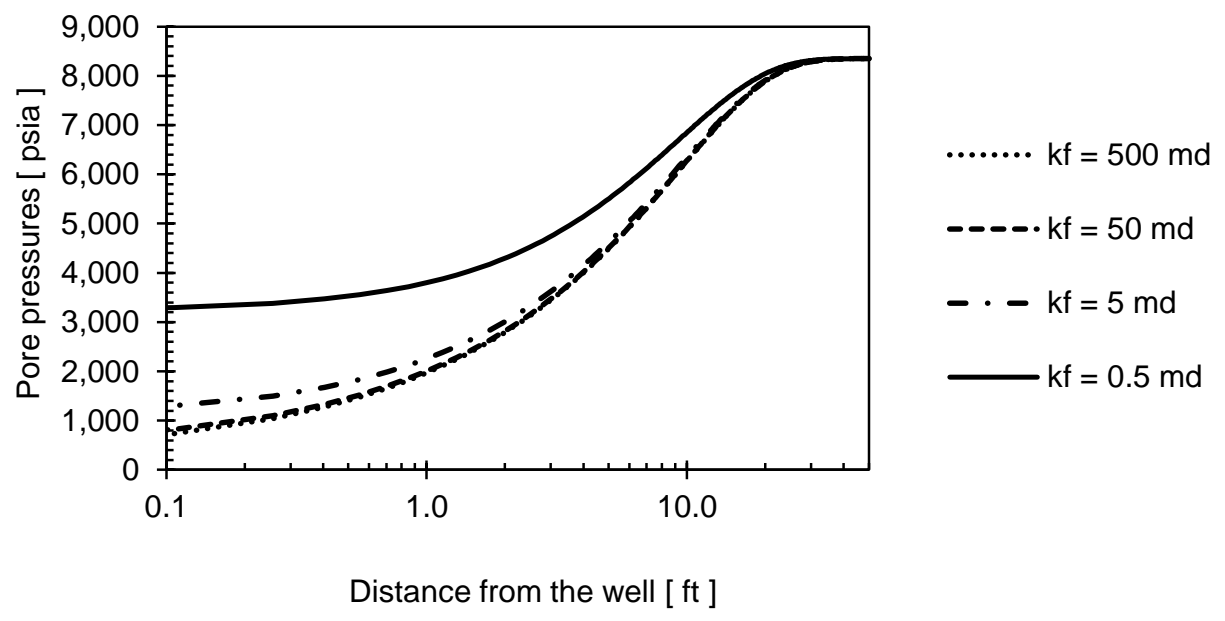

(b) Impact of fixed fracture permeability on cumulative production

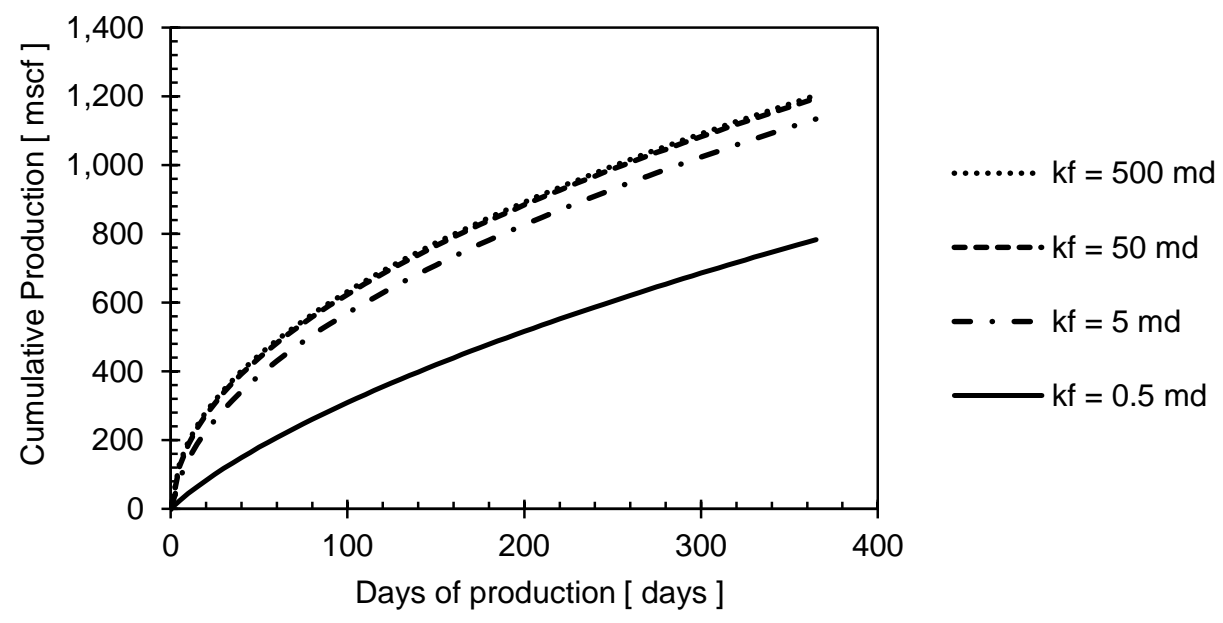

Figure 3.1. Results for the $k_{f}$ sensitivity runs (1D) 
This result is consistent with the previous work done by Wattenbarger et al. (1998). They derived productivity index expressions for a well with a planer fracture whose dimensionless fracture conductivity, $C_{f d}$, is infinite. The expressions are for the boundary condition of either constant rate or pressure. Since productivity of such a well is bounded, productivity index for a well with finite conductivity fracture should also be bounded. And the productivity index should reach the value of infinite conductivity case as the conductivity increases. A dimensionless fracture conductivity is defined as follows. In the equation, $k_{f}$ is the fracture permeability, $w$ is fracture width, $k$ is matrix permeability, and $X_{f}$ is fracture half length.

$$
C_{f d}=\frac{k_{f} w}{k X_{f}}
$$

Wattenbarger et al. (1998) mention that it could be assumed as an infinite conductivity if $C_{f d}>50$. Figure 3.1 is consistent in terms of having a threshold where $C_{f d}$ can be treated as an infinite conductivity. However, the threshold observed in Figure 3.1 is $C_{f d} \cong 2,000$, and the corresponding fracture permeability is $50 \mathrm{md}$. If fracture permeability is a parameter which deteriorates with time, for example, the effect will show up when the value becomes lower than the threshold. 


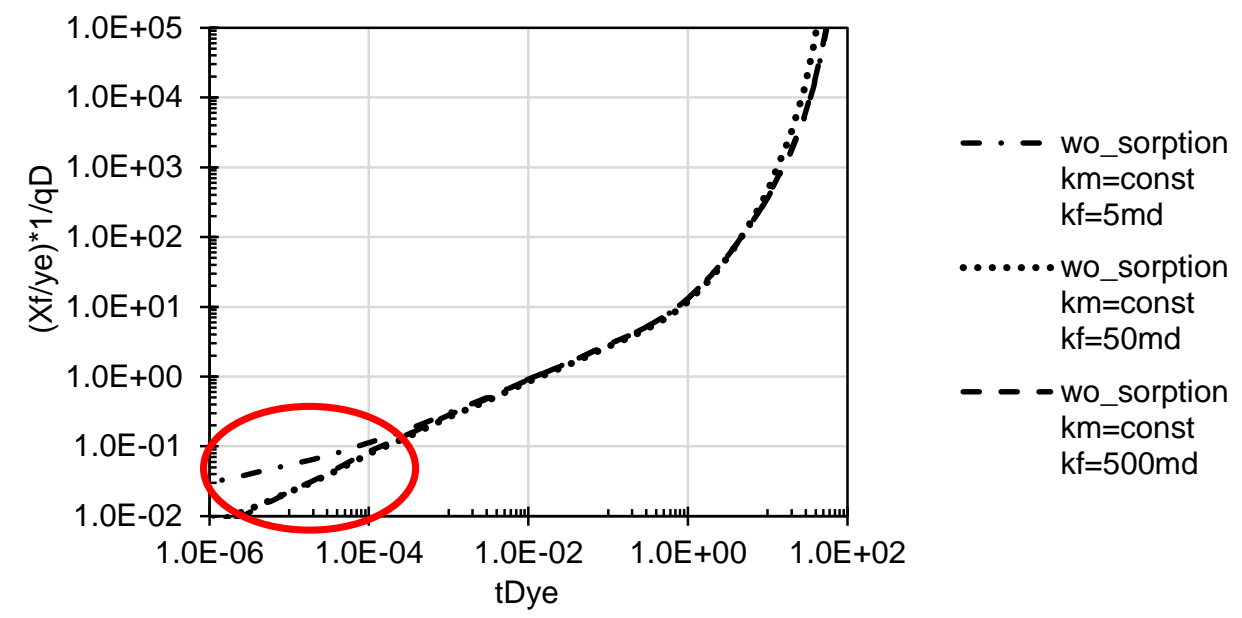

Figure 3.2. Production analysis on a shale reservoir featuring the fracture permeability

Fracture permeability below the threshold as a deviation from infinite conductivity assumption can be captured by using production analysis introduced in previous Section 2.5. Here, Figure 3.2 shows three curves whose fracture permeability differs. The dash dot line is when the fracture permeability is $5 \mathrm{md}$, the dot line is when the fracture permeability is $50 \mathrm{md}$, and the dashed line is when the fracture permeability is $500 \mathrm{md}$. For all the cases, sorption is not included, and the matrix permeability is fixed. In early time, less than $t_{D y e}=1$, half slope is seen for the dot line and dashed line. However, the dash dot line shows deviation from the half slope, which looks like a skin effect.

Even though the assumption for the fracture permeability is constant and simple, the case is informative, and give some insight before applying a stress sensitive fracture permeability model. Here, if configuration in the flow simulation is changed from 1D to 2D, then pressure distribution along the fracture could be obtained. Figure 3.3 shows the 
maximum pressure (solid line) and the minimum pressure (dashed line) in the fracture for each fracture permeability case. When the fracture has high enough permeability, for example Figure 3.3(c) or (d), the pore pressure in the fracture is almost uniform and does not change much with time. However, if the permeability is not high enough, then there will be larger pressure gradient and the pressure distribution changes more significantly as time proceeds.

From this observation it is expected that stress sensitive fracture permeability can become negligible if the fracture permeability is high enough. For this case, time dependent permeability declines under constant effective stress can be more important. However, the stress sensitive permeability might be able to make difference when the fracture permeability for given range of effective stress is below $50 \mathrm{md}$. Then the pore pressure will change more significantly over time which could result in reasonably large decline in fracture permeability due to change in effective stress. 
(a) $k_{f}=0.5 \mathrm{md}$

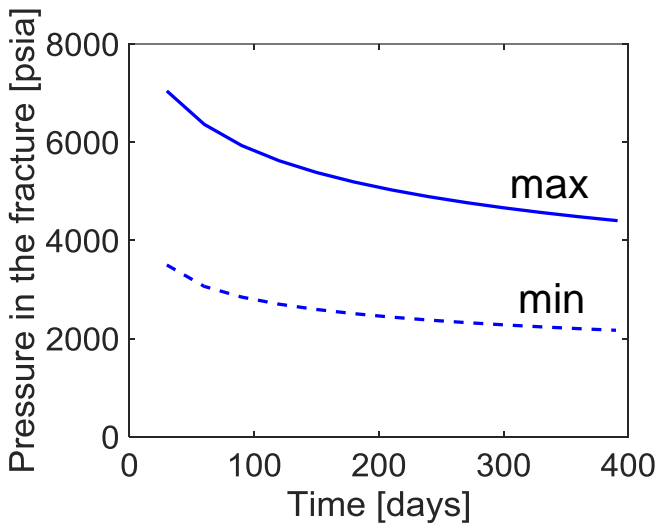

(c) $k_{f}=50 \mathrm{md}$

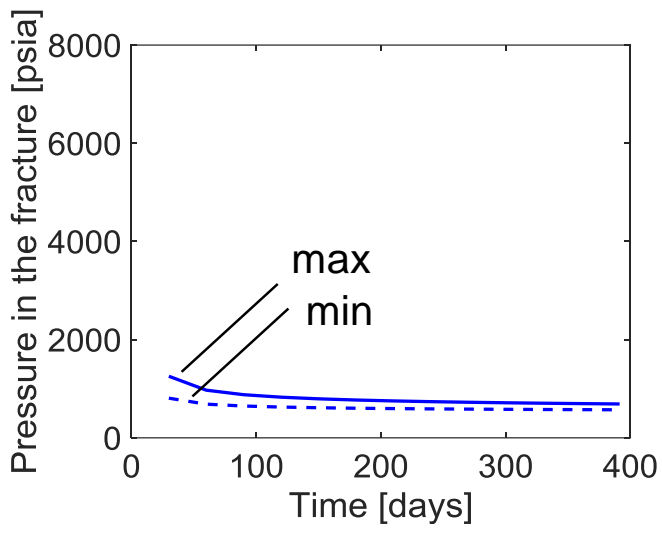

(b) $k_{f}=5 \mathrm{md}$

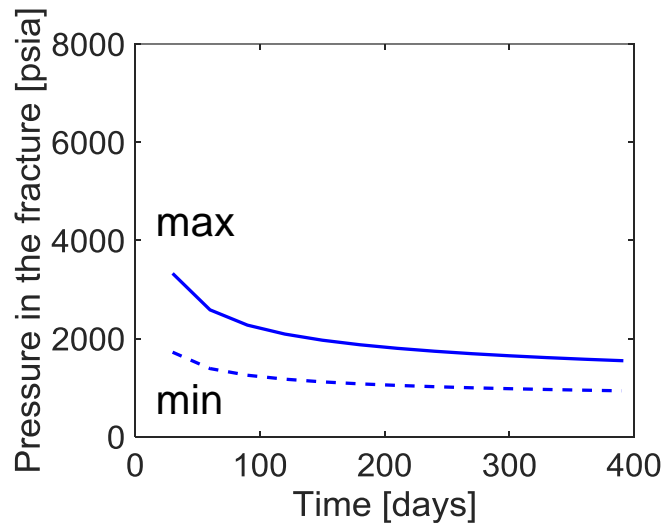

(d) $k_{f}=500 \mathrm{md}$

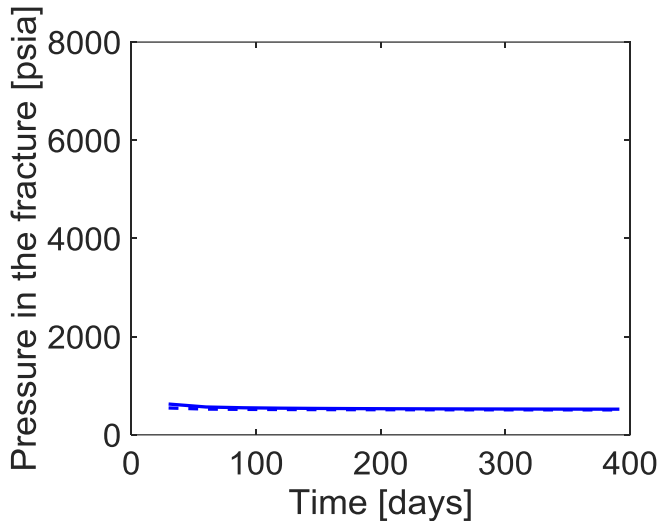

Figure 3.3. Max/Min pressure profile in the fracture 
In the previous section, the fracture permeability was fixed at $50 \mathrm{md}$ and a tornado chart showed the most influential mechanisms on production from shale matrix. This is the case when the fracture permeability is on the threshold, where fracture conductivity can be taken as infinite. What would happen if the fracture permeability is below the threshold? This case could be explained as a case when the fracture conductivity decreases with time no matter if the initial fracture permeability is above the threshold. Or it could be a case when the fracture conductivity is below the ideal value from the beginning.

Here, fracture permeability is changed from $50 \mathrm{md}$ to $5 \mathrm{md}$ to investigate how the fracture permeability would affect the production. Again, the eight parameters in the apparent matrix permeability model are perturbed and its effect on cumulative gas production is shown in the tornado chart in Figure 3.4. In the figure, bars from Figure 2.18 $\left(k_{f}=50 \mathrm{md}\right.$ case) are shown with white filling, and the case for $k_{f}=5 \mathrm{md}$ is shown with red or blue filling. In the figure you see more influence coming from stress sensitive permeability, and less influence from sorbed phase transport and pore diffusion compared with the case of $k_{f}=50 \mathrm{md}$. For example, the length of the bars increase by $6 \%$ for $p_{1}$ and $m$. As for $V_{s L}$ it decreases by $17 \%$ and $21 \%$ for $\varepsilon_{k s}$. 


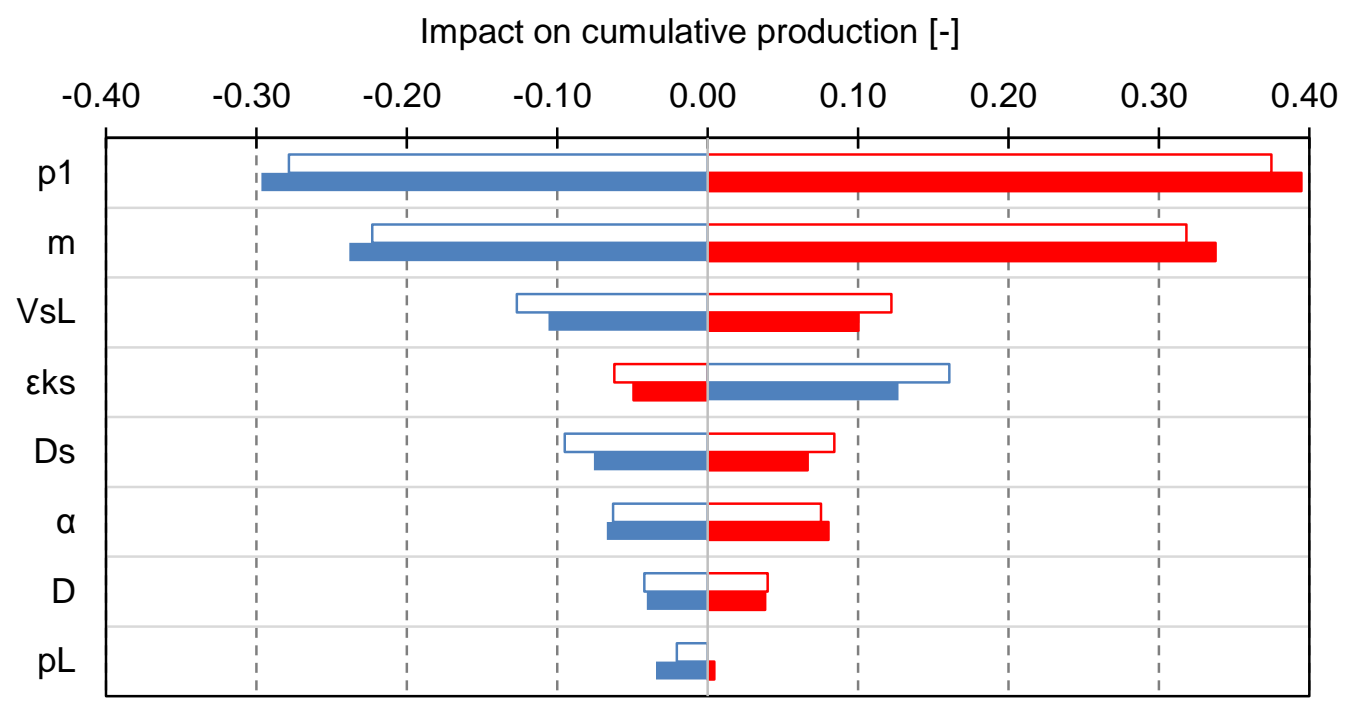

Figure 3.4. Sensitivity analysis of apparent permeability parameters on production ( $k_{f}=5$ md colored case compared with $k_{f}=50$ md uncolored case)

In order to explain the result, we go back to the cases where we have static fracture permeability and static matrix permeability. Figure 3.5(b) shows the pressure distribution after 1 year. This figure is showing the cross section perpendicular to the fracture. The fracture is on the left. The dashed line shows the result for $k_{f}=5 \mathrm{md}$, and the dotted line shows the result for $k_{f}=50 \mathrm{md}$. The case of $k_{f}=5 \mathrm{md}$ shows higher pressure distribution around the well compared to the other case. If you take a look at the apparent matrix permeability plotted in Figure 3.5 (a), you can see that these two different fracture permeability cases would result in different apparent matrix permeability. Therefore, if the fracture is not permeable enough, then the pore pressure in the adjacent matrix to the fracture would become higher and it will become harder to make use of the benefit coming from molecular transport effects. 
(a) Apparent matrix permeability

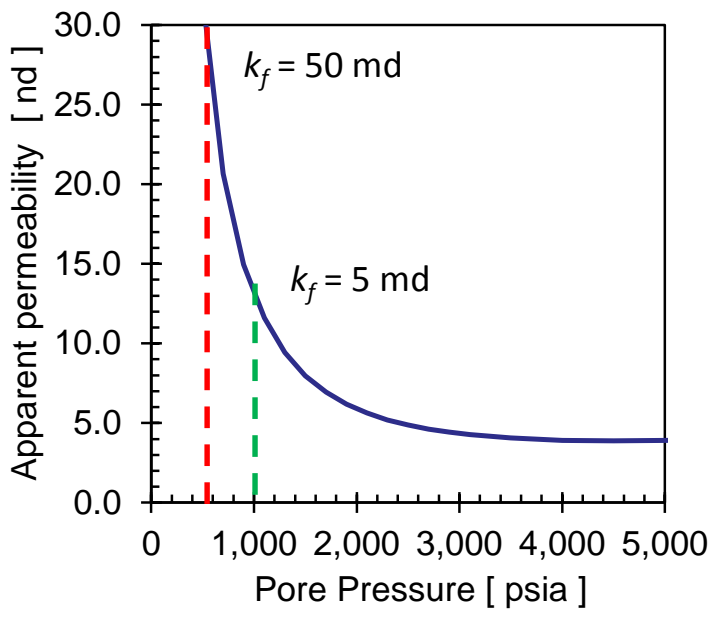

(b) Pore pressure distribution @ 1 year

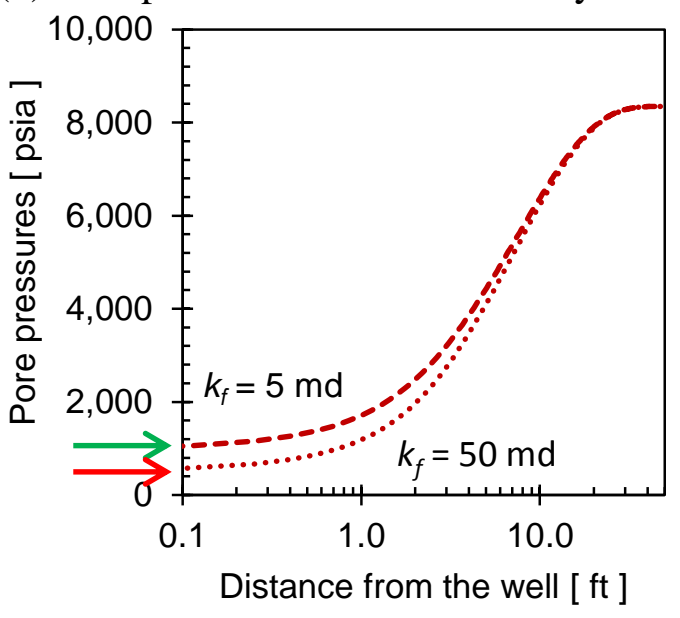

Figure 3.5. Corresponding apparent matrix permeability to pore pressure distribution after 1 year of production

One year cumulative production variance is computed here as well. This analysis is exactly the same as the one in Section 2.5. This time, the fracture permeability is set at $k_{f}=5 \mathrm{md}$. A triangular distribution is given for each of the eight parameter which appears in Equation (2.28), based on the range shown in Table 2.3 in Section 2.3. 500 sets of parameters are drawn randomly from these cumulative density functions, and 500 simulation runs are made to obtain one year cumulative production for each set. Table 3.1 shows the mean and variance of the 500 cumulative productions. Case 1 is when all the eight parameters in Equation (2.28) are perturbed. Case 2 is when the parameters from the first term, the stress sensitive term, are perturbed. Case 3 is when the parameters in the third term, the sorbed phase transport term, are perturbed. And Case 4 is when the parameter in the second term, the pore diffusion term, is perturbed. The variance becomes 
small for Case 3 and 4, which indicates the uncertainty, again, mainly comes from the stress sensitive term. Compared with the previous section, Table 2.5, the tendency does not change much. The largest uncertainty comes from the stress sensitive term, and the least comes from the pore diffusion term. Unlike Figure 3.4, it is not clear if the significance of the stress sensitive term is increasing or not. By taking the ratio of variances in Table 3.1, the ratio of Case 4 to Case 1 and that of Case 3 to Case 1 is decreasing compared to Table 2.5. Probably this indicates that the influence coming from the pore diffusion and the sorbed phase transport is decreasing as the fracture permeability, $k_{f}$, goes below the threshold. However, the ratio of Case 2 to Case 1 also decreases, and it is hard to say from Table 3.1 that the stress sensitive term becomes more influential when $k_{f}$ is below the threshold.

Table 3.1. Effect on uncertainty coming from each flow mechanism in shale matrix $\left(k_{f}=5 \mathrm{md}\right)$

\begin{tabular}{|l|r|r|}
\hline & $\begin{array}{c}\text { Mean } \\
(\text { mmscf })\end{array}$ & $\begin{array}{c}\text { Variance } \\
\left(\mathrm{mmscf}^{2}\right)\end{array}$ \\
\hline Case 1 (all of the eight parameters perturbed) & 1.24 & $8.08 \times 10^{-2}$ \\
\hline Case 2 (only the stress sensitive term perturbed) & 1.25 & $6.73 \times 10^{-2}$ \\
\hline Case 3 (only the sorbed phase transport term perturbed) & 1.22 & $5.19 \times 10^{-3}$ \\
\hline Case 4 (only the pore diffusion term perturbed) & 1.24 & $4.11 \times 10^{-4}$ \\
\hline
\end{tabular}




\subsection{Flow Mechanisms in a Hydraulic Fracture and Its Impact on the Production}

\subsubsection{Stress Sensitive Fracture Permeability}

Many researchers have looked into the stress sensitive elastic (reversible) behavior for hydraulic fracture permeability. Among all, two papers, Fredd et al. (2001) and Zhang et al. (2014), are reviewed to build a stress sensitive fracture permeability for this work. In both papers, the declining trend of fracture conductivity with increasing effective stress is shown as a function of proppant strength, and the proppant concentration in the fracture. In addition, the initial permeability can be controlled by the proppant grain size and the concentration. Looking at the figures in the literature, the data is fitted with an exponential curve to take into a flow simulation.

The literature gives an idea on fracture conductivity as a function of effective stress. However, the easiest way to put the stress sensitive conductivity to a flow simulation is to use stress sensitive permeability. Figure 3.6 shows an example of stress sensitive fracture permeability model. The permeability model needs to be a direct function of pore pressure for easy use. Then two steps are required to obtain a stress sensitive permeability model. One is conversion from effective stress to fluid pressure in a hydraulic fracture. Effective stress, $p_{e}$, is calculated by $p_{e}=p_{c}-\alpha \cdot p \cdot p_{c}$ is confining stress, for which minimum horizontal stress is used in many cases. $\alpha$ is Biot coefficient, which is unity for a hydraulic fracture. This is a common and reasonable assumption for a hydraulic fracture since it is a highly porous media. And $p$ is fluid pressure. In this way, fracture conductivity could be interpreted as a function of fluid pressure. The other step is 
conversion from fracture conductivity to fracture permeability. Fracture conductivity is a product of fracture width and fracture permeability. It is likely that the width is changing as effective stress increases, however, it is assumed to be constant and, instead, only permeability is changing. Finally, a permeability model for a hydraulic fracture could be given as an exponential function of pore pressure as shown in Figure 3.6.

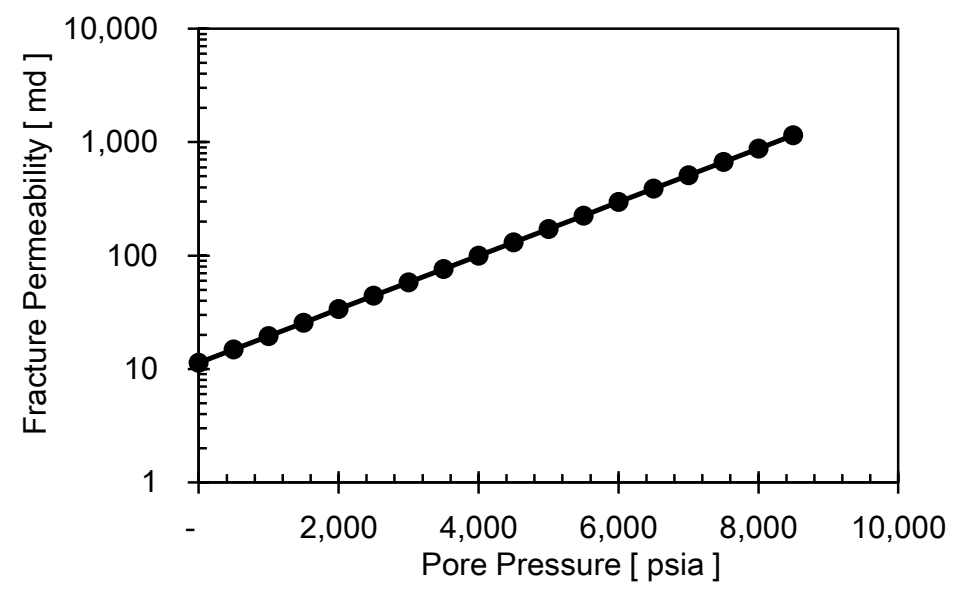

Figure 3.6. Stress sensitive fracture permeability model [modified from Zhang et al. (2014)] 


\subsubsection{Time-dependent Fracture Permeability}

There is another stress sensitive response which is sometimes called "creep." This is time dependent response under constant stress condition. On the other hand, the stress sensitivity discussed in the previous section was instantaneous response. Creep is an inelastic deformation often discussed in literature on rock mechanics. It is often described with three regimes (Figure 3.7), (i) transient creep, (ii) steady-state creep, and (iii) accelerating creep. In this figure, the strain, $\varepsilon$, shown on $y$-axis is, for example, a deformation of the rock over original sample length. The time-strain relationship is often expressed with the following formulation [Jaeger et al. (2007)]:

$$
\varepsilon=\varepsilon_{e}+\varepsilon_{1}(t)+V t+\varepsilon_{3}(t)
$$

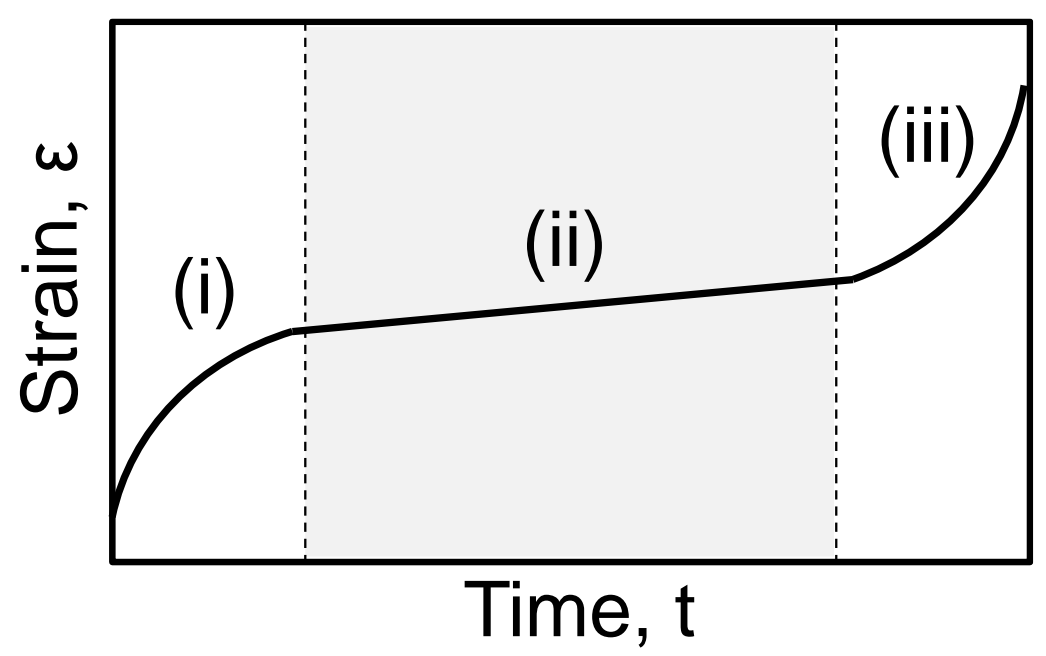

Figure 3.7. Idealized creep curve with three regimes [modified from Schwartz and Kolluru (1981)] 
$\varepsilon_{e}$ is the instantaneous elastic strain, $\varepsilon_{l}(t)$ corresponds to the regime (i), where strain rate $(\dot{\varepsilon}=d \varepsilon / d t)$ or the gradient in Figure 3.7 decreases with time. Until the first transient creep, the deformation is often described as viscoelastic and assumed reversible. $V \cdot t$ corresponds to the regime (ii), where strain rate is constant. $\varepsilon_{3}(t)$ corresponds to the regime (iii), where the strain rate increases in time just before the failure. This discussion is mainly for intact rock. However, probably there is no intact rock in the reality. Schwartz and Kolluru $(1981,1982)$ attempt to look into the effect coming from discontinuity. They conducted creep measurements on jointed rock samples, and showed the joint could result in additional creep strain. This is caused by stress concentration at joint asperities (Figure 3.8). This stress concentration could occur in a hydraulic fracture at the contact of fracture face and proppant to cause additional joint creep within the fracture. If this phenomenon from rock mechanics is taken and applied to understand the dynamics in a hydraulic fracture, the analogy is proppant embedment.

(a)

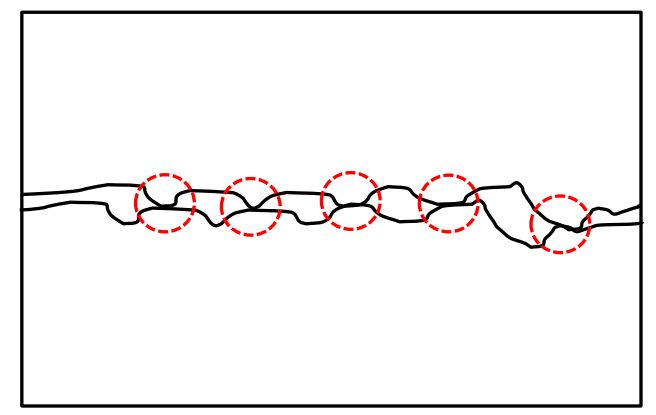

(b)

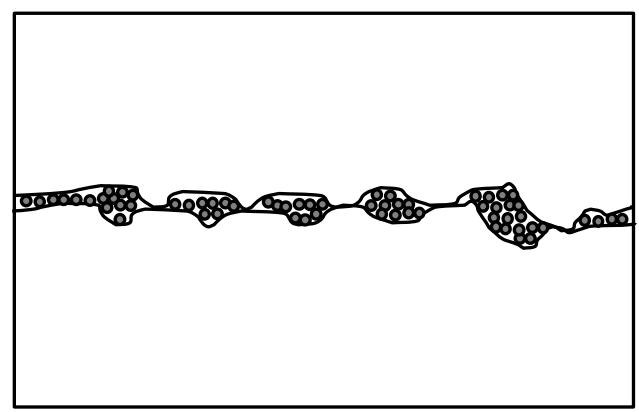

Figure 3.8. Stress concentration at (a) joint asperities, (b) proppant 
Based on the conceptual discussion above, the following assumptions are introduced for a hydraulic fracture to construct a time-dependent permeability model for the porous media.

- Constant stress applied for the entire production period.

- The fracture width, $w_{o}$, becomes narrower due to proppant embedment, $w_{o}(1-\varepsilon)$, following the logarithmic function in Equation (3.3).

$$
\varepsilon=a \ln (b t+1)
$$

- Instead of introducing time varying fracture width, assume constant width, $w_{o}$, and introduce time varying fracture permeability shown in Equation (3.4)

$$
k_{f}=k_{f, \text { init }}(1-\varepsilon)
$$

$\left[\because(\right.$ Fracture conductivity $\left.)=k_{f, \text { init }} w_{o}(1-\varepsilon)\right]$

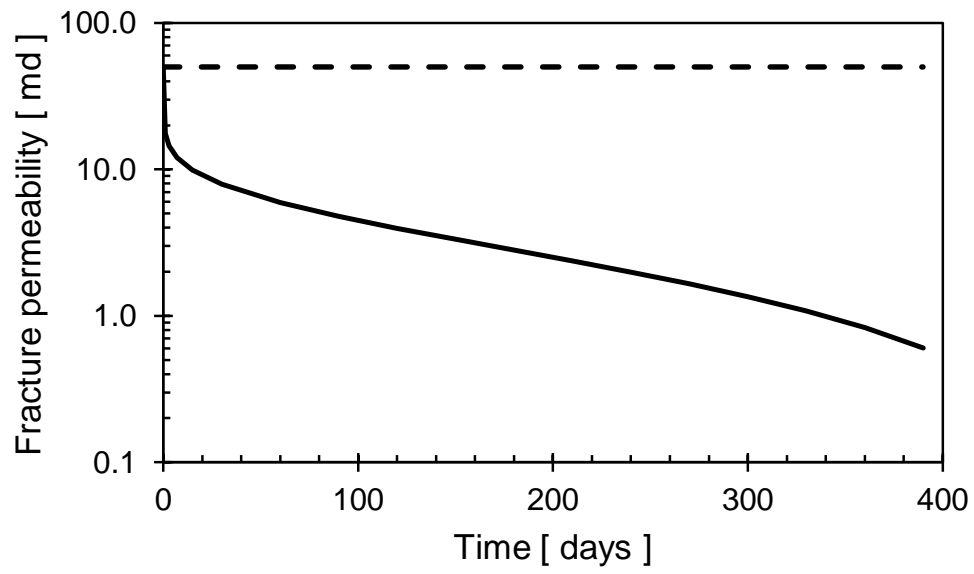

Figure 3.9. Time deteriorating fracture permeability $\operatorname{model}(a=0.057 ; b=1)$ 
Note that although Equation (3.3) assumes regime (i), proppant embedment is irreversible deformation. These assumptions require the condition of constant effective stress over the production time. If the fracture permeability is high enough, then the high fluid pressure in the fracture prevails only in a short production time (this is already shown in in Section 3.1), and the fracture permeability will be controlled by the bottom-hole pressure. In that case, constant stress condition can be valid. As for the time-strain relation shown in Equation (3.3), similar relation is also shown in Schwartz and Kolluru (1981), and Wen et al. (2007). Figure 3.9 shows one extreme example of time dependent fracture permeability constructed based on the assumptions above. The dashed line shows constant fracture permeability of $50 \mathrm{md}$, and the solid line shows time dependent fracture permeability. The solid line also starts from $50 \mathrm{md}$, however, deteriorates with time. The parameters selected to feed into the time dependent permeability model in Equation (3.4) is also shown with the figure. With this parameters, the width of a hydraulic fracture keep decreasing and completely closes after one and a half year.

\subsubsection{Forchheimer Effect in a Hydraulic Fracture}

Forchheimer effect is a deviation from Darcy's law at high fluid flow rate found by Forchheimer in 1901. So-called Forchheimer equation has an additional pressure drop term to Darcy's flow equation which is a function of flow rate [Equation (3.5)]. Because of this term, apparent permeability at a given flow rate becomes smaller than that based on Darcy's law. There has been a lot of discussion to give a physical explanation on this additional pressure drop term. The most common explanation on this additional pressure 
drop is "inertial effect" [ $\mathrm{Li}$ and Engler (2001)]. In other words, because the flow paths in porous media are twisted and winding, the flow becomes a vector whose components can be split into two, the portion that contributes to the fluid flow through the path, and the portion that does not contribute to the transport. This inefficiency increases non-linearly with larger flow rate, which is the so-called inertial or inertia effect [Ruth and Ma (1992)].

$$
-\frac{\partial p}{\partial x}=\frac{\mu v}{k_{D}}+\beta \rho v^{2}
$$

$\mu$ is the fluid viscosity $(\mathrm{Pa} \cdot \mathrm{s}), v$ is the average velocity of the fluid $(\mathrm{m} / \mathrm{s}), k_{D}$ is permeability without non-Darcy's effect $\left(\mathrm{m}^{2}\right), \beta$ is so-called Forchheimer coefficient $(1 / \mathrm{m})$ shown below, and $\rho$ is the fluid density $\left(\mathrm{kg} / \mathrm{m}^{3}\right)$. Several models for Forchheimer coefficient has been proposed by different researchers, and this is well-summarized by $\mathrm{Li}$ and Engler (2001). One of the model is shown in Equation (3.6). This could be derived by combination of modified Ergun Equation (3.7) in Macdonald et al. (1979) and BlakeKozeny Equation (3.8). Also one of the models shown in Li and Engler (2001) as Kutasov model for the case when there is no water saturation. Here, $N_{R E}$ is Reynolds number, $N_{R E}$ $=\rho v D_{p} / \mu$. Here, $D_{p}$ is grain diameter.

$$
\begin{aligned}
& \beta \cong \frac{0.143}{k_{D}^{0.5} \phi^{1.5}} \\
& -\frac{\partial p}{\partial x}=\frac{1}{2}\left(1.75+\frac{150(1-\phi)}{N_{R E}}\right) \frac{1-\phi}{\phi^{3}} \frac{2 \rho v^{2}}{D_{p}} \\
& k_{D}=\frac{D_{p}^{2} \phi^{3}}{150(1-\phi)^{2}}
\end{aligned}
$$


By applying Equation (3.8) to Equation (3.7), Equation (3.5) could be derived with Forchheimer coefficient $\beta=1.75(1-\phi) /\left(\phi^{3} D_{p}\right)$. And again, apply Equation (3.8) will give the model shown in Equation (3.6).

Now, take Equation (3.5) and reorganize it. An effective permeability that could be substituted in Darcy's equation will be derived, Equation (3.9).

$$
\begin{gathered}
-\operatorname{grad}(p)=\frac{\mu v}{k_{D}}+\beta \rho v^{2} \\
\Leftrightarrow-\operatorname{grad}(p)=\frac{\mu v}{k_{D}}\left(1+\frac{k_{D} \beta \rho v}{\mu}\right) \\
\Leftrightarrow-\operatorname{grad}(p)=\frac{\mu v}{k_{D} /\left(1+\frac{k_{D} \beta \rho v}{\mu}\right)} \\
k_{N D}=\frac{k_{D}}{1+\frac{k_{D} \rho v \beta}{\mu}} \ldots \ldots \ldots
\end{gathered}
$$

Note that " $v=-k_{N D} / \mu \cdot \operatorname{grad}(p)$ " then Equation (3.9) will be recognized as a quadratic equation for $k_{N D}$ and it could be used for $k_{N D}$. By solving this quadratic equation, Equations (3.10) and (3.11) are derived. These derived equations are uses in a flow simulation later on to investigate the Forchheimer effect on production.

$$
\begin{array}{r}
k_{N D}=\frac{1}{2 X}+\sqrt{\left(\frac{1}{2 X}\right)^{2}-\frac{k_{D}}{X}} \\
X=\frac{k_{D} \rho \beta}{\mu^{2}}\left(\frac{\partial p}{\partial x}\right) \ldots \ldots
\end{array}
$$


In order to visualize the difference between Darcy's permeability, $k_{f D}$, and the one with Forchheimer effect, $k_{f N D}$, the following plot in Figure 3.10 is prepared. $k_{f D}$ corresponds to $k_{D}$ in Equation (3.10). The subscript of " $f$ " is added to clarify that it is fracture permeability. The same rule applies to $k_{f N D}$. This corresponds to $k_{N D}$ in Equation (3.10). The subscript " $f$ " indicates that it is fracture permeability, and " $N D$ " indicates that this is effective permeability when non-Darcy flow, in this case Forchheimer effect, is considered. This plot shows fracture permeabilities against pore pressures. Since permeability with Forchheimer effect is a function of pore pressure and its gradient, several $k_{f N D}$ with different pressure gradients are shown. Here, the permeability without Forchheimer effect is $k_{f D}=50 \mathrm{md}$. Suppose a linear pressure gradient across a hydraulic fracture whose half-length of $175 \mathrm{ft}$, the bottom-hole pressure of 500 psia, pore pressure at the fracture tip 8,350 psia would give a pressure gradient around $10^{6} \mathrm{~Pa} / \mathrm{m}$. The third dashed line from the bottom in Figure 3.10 corresponds to this pressure gradient. Looking at Figure 3.10, it is expected that especially in the early stage of production, Forchheimer effect could pose a negative effect on production, and it is worth examining this effect by applying this permeability model in a flow simulation. 


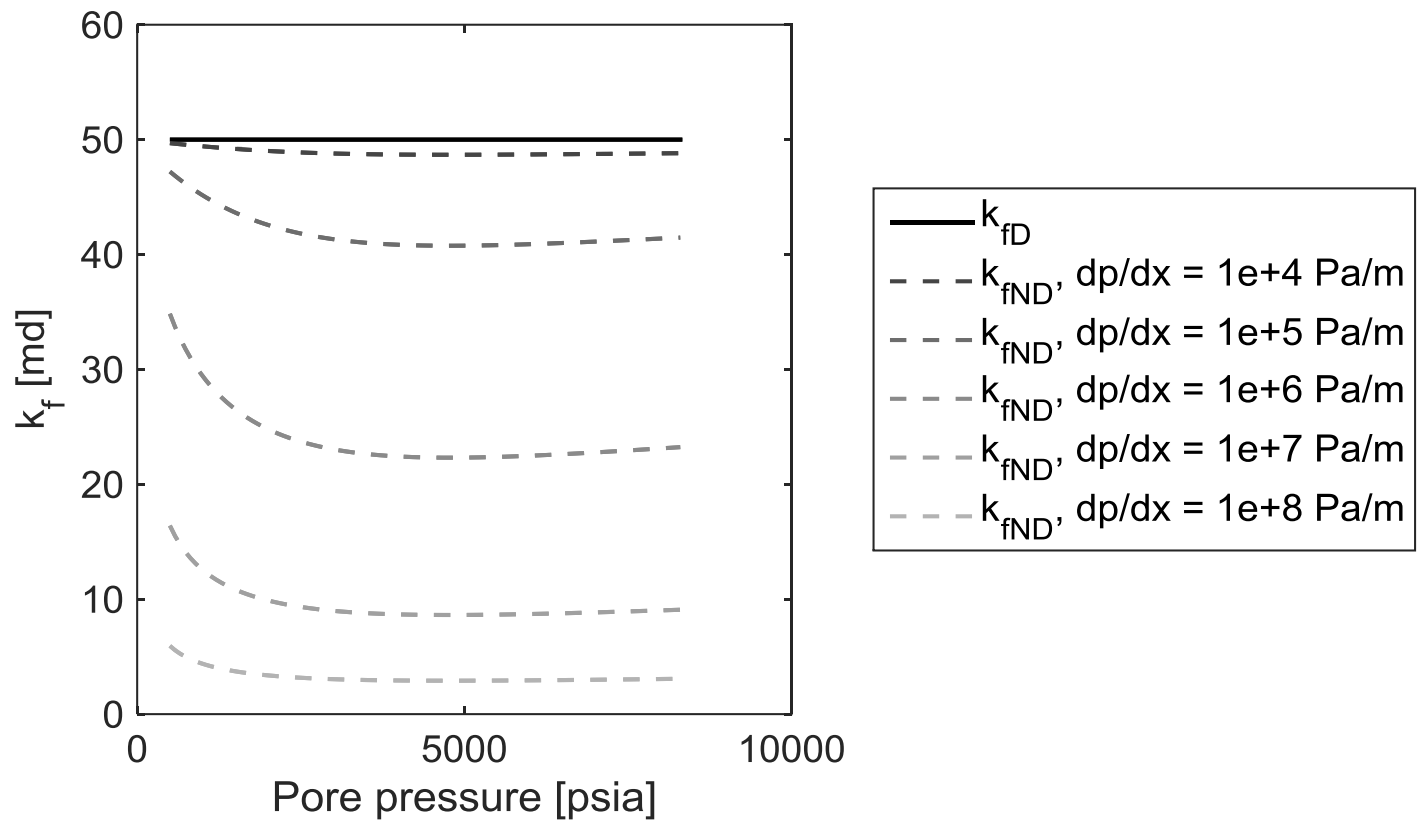

Figure 3.10. Fracture permeabilities with Forchheimer effect

Looking at Figure 3.10, now we take the Forchheimer effect in the flow simulation and see if it will affect the production. Using fracture permeability of 5, 50, and $500 \mathrm{md}$, both cases with Forchheimer effect and without Forchheimer effect are compared. For this simulation run, Forchheimer coefficient, $\beta$, is required. Equation (3.6) is used in this work, assuming porosity in the fracture as 0.3 and Darcy's permeability (permeability without Forchheimer effect) of 5, 50, and 500 md respectively. Corresponding Forchheimer coefficients are 12.23, 3.87, and $1.22 \mathrm{~atm}^{*} \mathrm{~s}^{2} / \mathrm{g}$ respectively. (For this computation, Eclipse is used rather than the in-house simulator because of computation time issue.) 


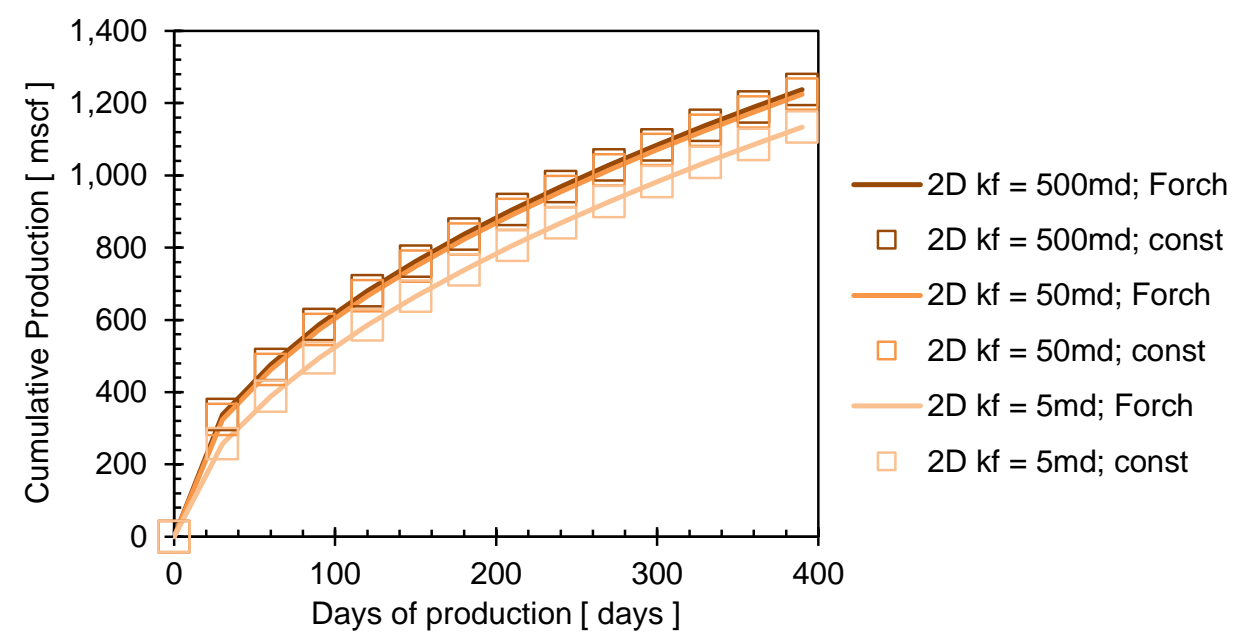

Figure 3.11. Forchheimer effect on production

Figure 3.11 shows the result of the total 6 runs. Solid lines show the cases with Forchheimer effect, and square markers show the cases without the effect. Negative effect on production is expected for the runs with Forchheimer effect, however, as you can see from Figure 3.11, that negative effect does not appear clearly.

The possible explanation for this result is that there is almost negligible difference between non-Darcy permeability (the one with Forchheimer effect, $k_{f N D}$ ) and Darcy permeability (the one without Forchheimer effect, $k_{f D}$ ). In order to check this, the average permeability profiles in the fracture are plotted in Figure 3.12. Left column in the figure shows pressure distribution along the fracture, from the well bore to the tip, and the figure shows how the pressure in the fracture evolves as time proceeds. Recall that the initial reservoir pressure is 8,350 psia. All the pressure distributions shown in Figure 3.12 indicates that the entire fracture is in the drainage volume. Therefore, the average permeability in the fracture can be calculated using all the cells in the fracture. Fracture 
permeability profiles are shown in the right column of Figure 3.12. The maximum, average, minimum fracture permeabilities are plotted. Average permeability is calculated as a harmonic average. The maximum fracture permeabilities, $k_{f, \max }$, are almost the same as Darcy permeability over time, and the minimum permeabilities, $k_{f, m i n}$, are always lower than the maximum values. However, the average permeabilities, $k_{f, a v e}$, are rather closer to the maximum values. In Figure 3.12 (a-2), it is almost impossible to differentiate $k_{f, \max }$ from $k_{f, a v e}$. This explains why the cumulative production did not change in Figure 3.11. The same explanation applies to Figure 3.12 (c-2) as well. And Figure 3.12 (c-2) can be explained by the discussion on the threshold. Previously we found that the threshold fracture permeability for our configuration is around $50 \mathrm{md}$, and the permeability values we see in Figure 3.12 (c-2) never go below the threshold. Therefore we did not observe difference in cumulative production in Figure 3.11. According to this observation, probably the Forchheimer effect in the fracture is not significant. 
(a-1) Pressure profile for $k_{f D}=5 \mathrm{md}$

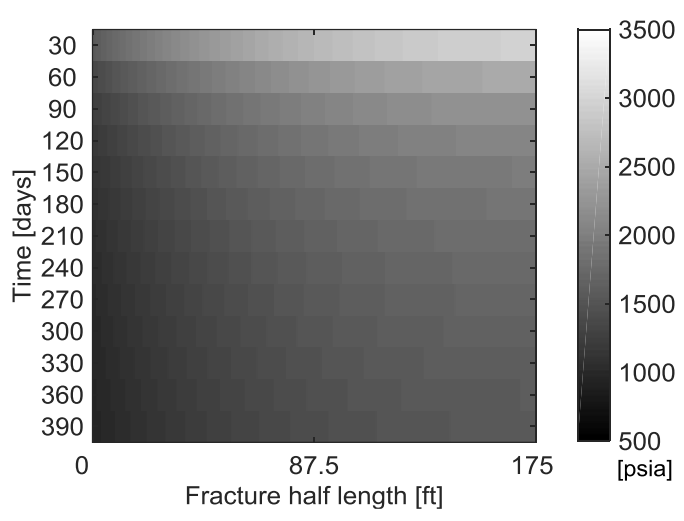

(b-1) Pressure profile for $k_{f D}=50 \mathrm{md}$

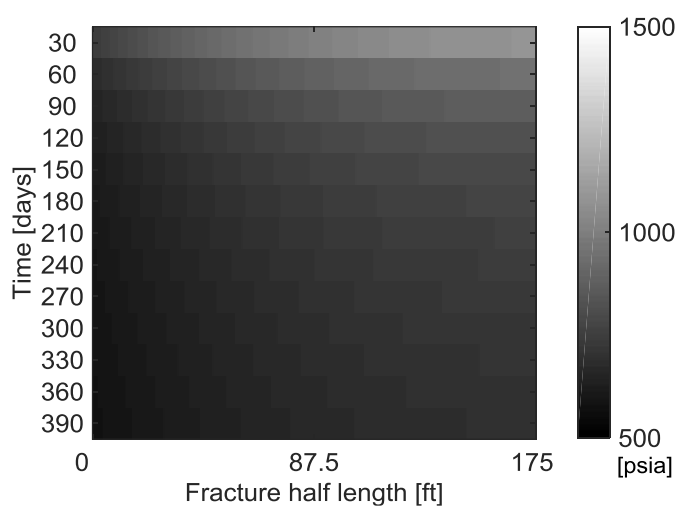

(c-1) Pressure profile for $k_{f D}=500 \mathrm{md}$

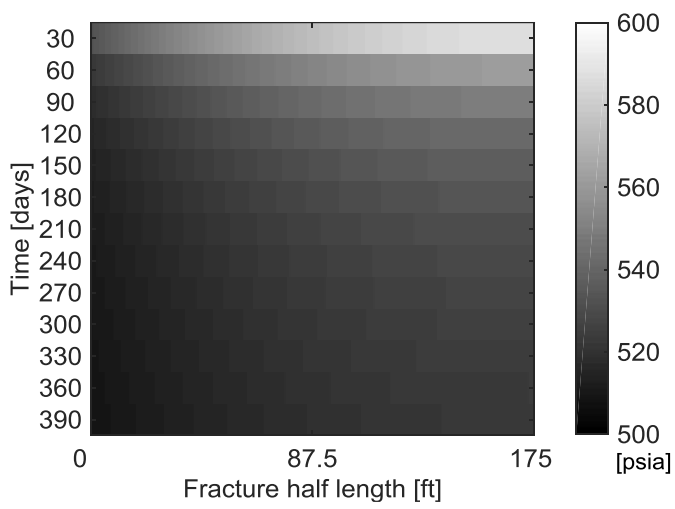

(a-2) $k_{f N D}$ profile for $k_{f D}=5 \mathrm{md}$

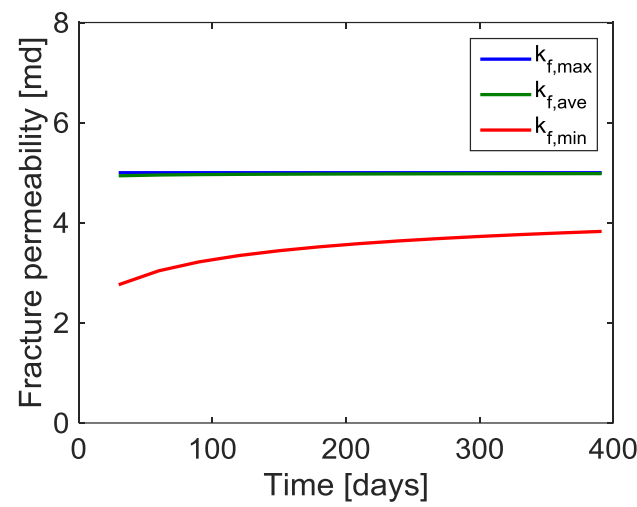

(b-2) $k_{f N D}$ profile for $k_{f D}=50 \mathrm{md}$

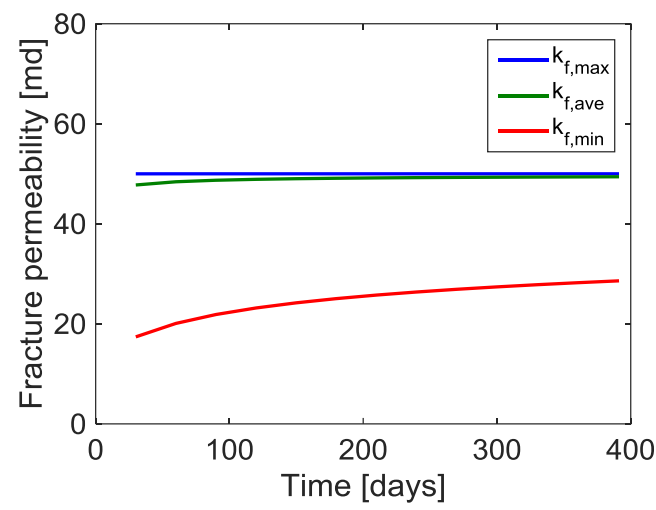

(c-2) $k_{f N D}$ profile for $k_{f D}=500 \mathrm{md}$

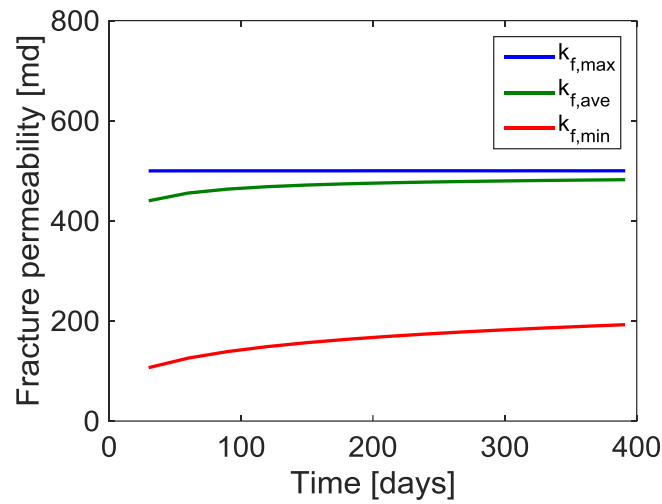

Figure 3.12. Fracture permeability, $\boldsymbol{k}_{f N D}$, and pressure profile 


\section{PLAUSIBLE EFFECT OF CHOKING A WELL}

In the industry, it is often said that cumulative production could be improved by choking a well. The explanation to this goes by looking at the Darcy's flow Equation (4.1), where $q$ is the flow rate and $A$ is a cross section area. $k$, is permeability and fluid viscosity is $\mu$, and pressure gradient of $d p / d x$. Although we will reduce $d p / d x$ by keeping the bottomhole pressure sufficiently high, we can prevent impairment in matrix permeability, $k$, because of elastic/inelastic stress sensitivity of the fracture permeability. The benefit from

maintaining the bottom-hole pressure high overcomes the negative effect from reducing the pressure gradient, and eventually realizes more cumulative production.

$$
\begin{array}{r}
q=-\frac{k A}{\mu} \frac{d p}{d x} \ldots \ldots \ldots \ldots \ldots \ldots \ldots \ldots \ldots \ldots \ldots \ldots \\
\frac{d q}{d p_{w f}}=\frac{\partial q}{\partial k} \frac{d k}{d p_{w f}}+\frac{\partial q}{\partial \mu} \frac{d \mu}{d p_{w f}}+\frac{\partial q}{\partial(d p / d x)} \frac{d(d p / d x)}{d p_{w f}} \\
\frac{d q}{d p_{w f}}=-\frac{A}{\mu}\left(\frac{d p}{d x} \frac{d k}{d p_{w f}}-\frac{k}{\mu} \frac{d p}{d x} \frac{d \mu}{d p_{w f}}+k \frac{d(d p / d x)}{d p_{w f}}\right)
\end{array}
$$

In a sense, if one take a derivative of Equation (4.1) with respect to bottom-hole pressure, $p_{w f}$, Equation (4.2) and (4.3) can be obtained. By assuming stress sensitive permeability in the drainage, we consider non-zero $d k / d p_{w f}$. Without stress sensitivity of the matrix permeability, $d k / d p_{w f}$. is zero. However, the choking effect is an idea that contradicts to this typical expectation. In the industry, those who believe in the choking effect expect positive $d q / d p_{w f}$ due to significant magnitude of $d k / d p_{w f}$. Similar discussion 
is found in RPSEA report by Higgs-Palmer Technologies (2013) as a "drawdown management."

In this section, the sector model with a single fracture is used to investigate the effect of choking the well. For this purpose, bottom-hole pressure is changed from 500 psia to 1,500 psia. First, with fixed fracture permeability with all the dynamics in shale matrix to see if there is any effect from the matrix. Then, fixed matrix permeability with dynamic fracture permeability is considered.

Let us go back to the first case of consideration. That is the dynamic matrix permeability with different bottom-hole pressures. The results are shown in Figure 4.1. Figure 4.1(a) shows the static permeability case. Figure 4.1(b) is the case where only stress sensitive permeability is considered. Since stress sensitive permeability poses a negative effect on production, all cases shown in Figure 4.1(b) lead to less production compared to Figure 4.1(a). However, note that difference between low bottom-hole pressure case, 500 psia, and high bottom-hole pressure case, 1,500 psia, Figure 4.1(b) is small, almost negligible. This is because of the effect coming from keeping the bottomhole pressure high. This certainly helps to maintain the matrix permeability. However, this operation also leads to less drawdown which results in overall decrease in cumulative production. Figure 4.1(c) shows the case where all the three transport mechanisms, i.e., stress sensitive permeability, sorbed phase transport, and pore diffusion, are taken into account. Stress sensitive permeability is a negative effect, and molecular transport mechanisms are positive effects on the production. And Figure 4.1(c) shows overall apparent permeability improvement. When the difference between the bottom-hole 
(a) fixed matrix permeability

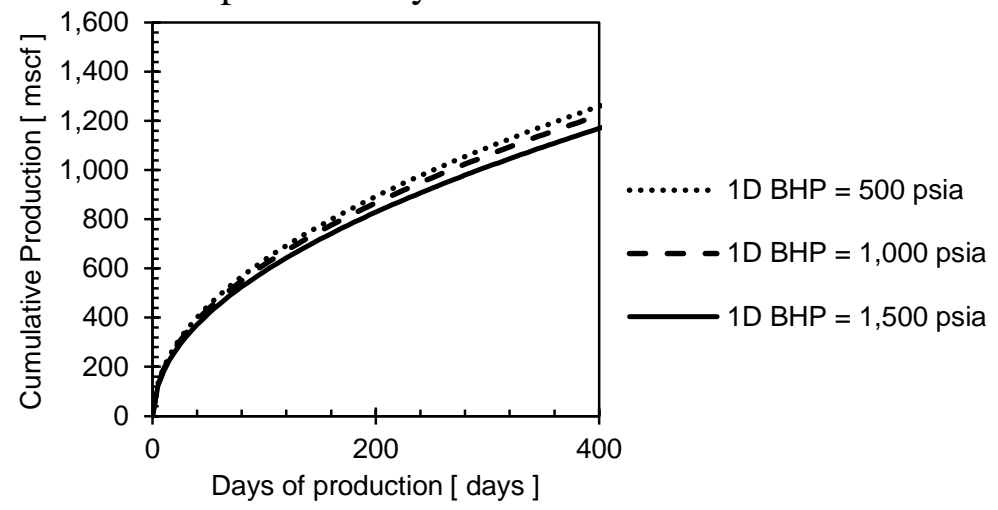

(b) Apparent permeability only with stress sensitive term

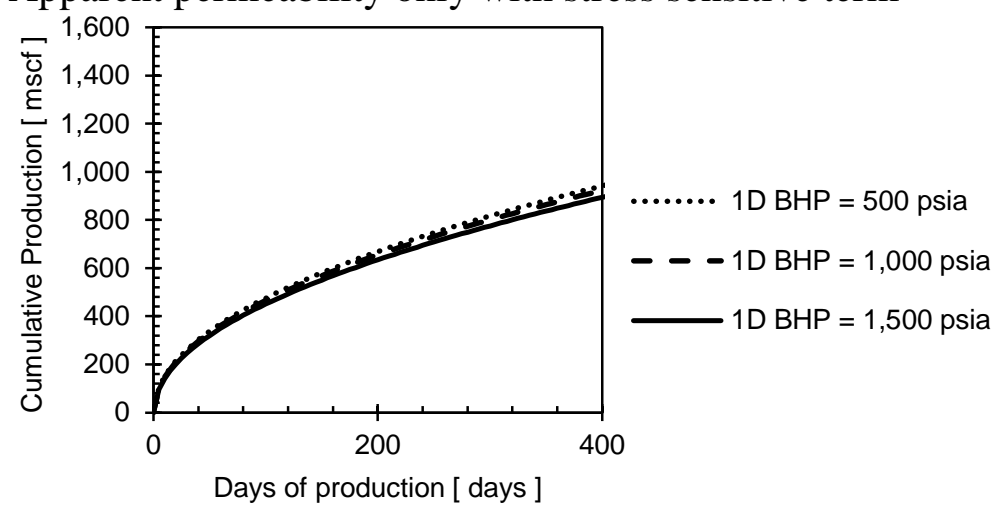

(c) Apparent permeability with all the three transport mechanisms

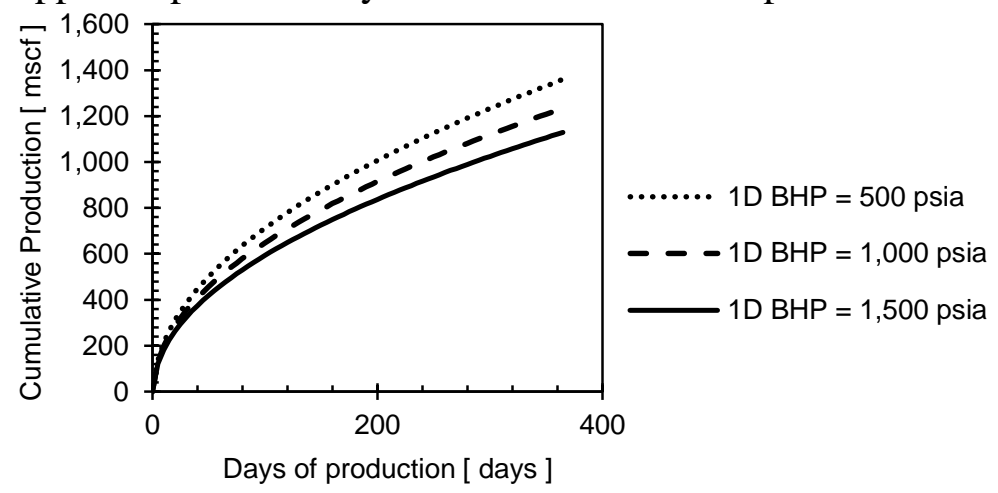

Figure 4.1. Choking effect on cumulative production (constant $\boldsymbol{k}_{f}$; dynamic $\boldsymbol{k}_{\text {matrix }}$ ) 
pressures is focused, Figure 4.1(c) shows the largest difference. This result is consistent with the one we saw in Section 3.1. In other word, less contribution from sorbed-phase transport is expected at high bottom-hole pressure. This leads to less cumulative production. None of the plots in Figure 4.1 shows choking the well helps with the production. From this observation, the so-called choking effect is very likely not coming solely from the matrix.

As a next step, dynamic fracture permeability is introduced under fixed matrix permeability. Three factors were listed previously, which affect the flow in a hydraulic fracture, i.e., stress sensitive reversible permeability, time dependent irreversible permeability, and the Forchheimer effect. In Section 3.2.3, it was shown that the Forchheimer effect is negligible even for the largest drawdown with the bottom-hole pressure of 500 psia. Therefore, in this section, we exclude the Forchheimer effect and focus to the other two effects.

First, stress sensitive reversible (or elastic) fracture permeability is considered. In this case, fracture permeability is treated as a function of pore pressure which declines as the pore pressure decreases. One possible reason which may lead to this effect is that a hydraulic fracture is not well-designed such that the conductivity goes below the minimum requirement (mentioned as "threshold" in Section 3.1) at the minimum bottom-hole pressure. Therefore in order to maintain the fracture conductivity, the bottom-hole pressure should be kept high enough. This is investigated by using the sector model. The fracture permeability models used in this investigation is shown in Figure 4.2. 


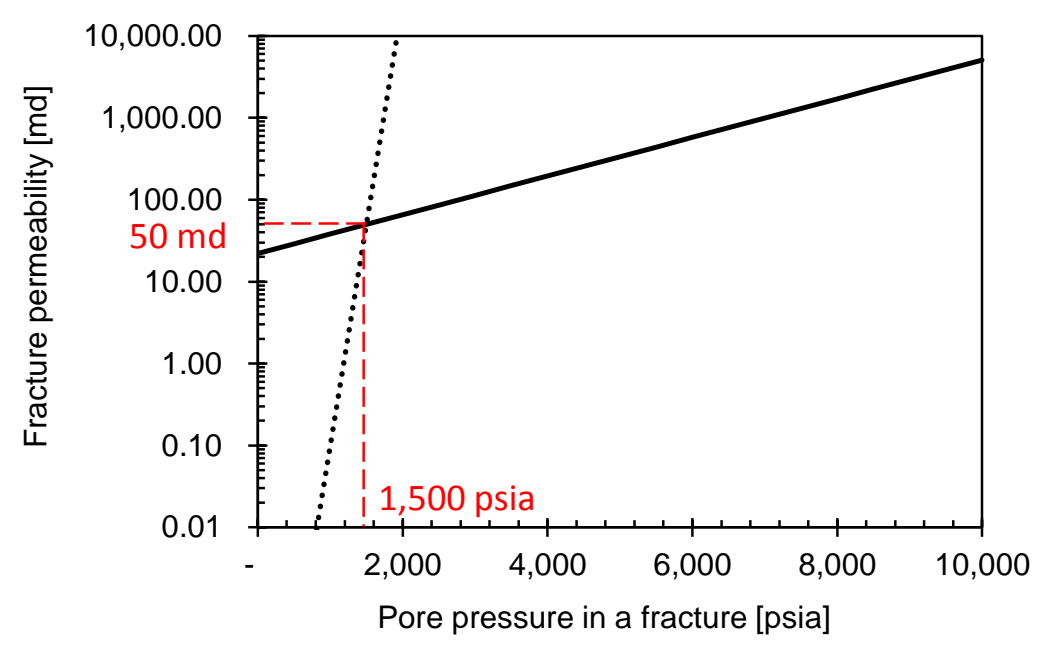

Figure 4.2. Stress sensitive fracture permeability models used

The solid line is constructed referring to Zhang, et al. (2014). The gradient corresponds to the ones in the paper with the condition of aligned, propped, 30/50 mesh, and proppant concentration of $0.1 \mathrm{lbm} / \mathrm{ft}^{2}$. The dotted line is an extreme case where the fracture permeability becomes comparable to that of matrix at pore pressure of 500 psia. Here, matrix permeability is fixed at 4.5 nd approximately. Both fracture permeability models show $50 \mathrm{md}$ at pore pressure of 1,500 psia, and three different bottom-hole pressure values, 500, 1,000, and 1,500 psia, are considered.

Figure 4.3 shows the resulting cumulative production curves. Figure 4.3(a) corresponds to the case of solid line in Figure 4.2, and Figure 4.3(b) corresponds to the dotted line in Figure 4.2, higher sensitivity to stress. In Figure 4.3, cumulative production decreases as stress sensitivity increases. And the difference in cumulative production decreases as well. However, choking a well does not result in more cumulative production in any of the cases. 
(a) Stress sensitive fracture permeability

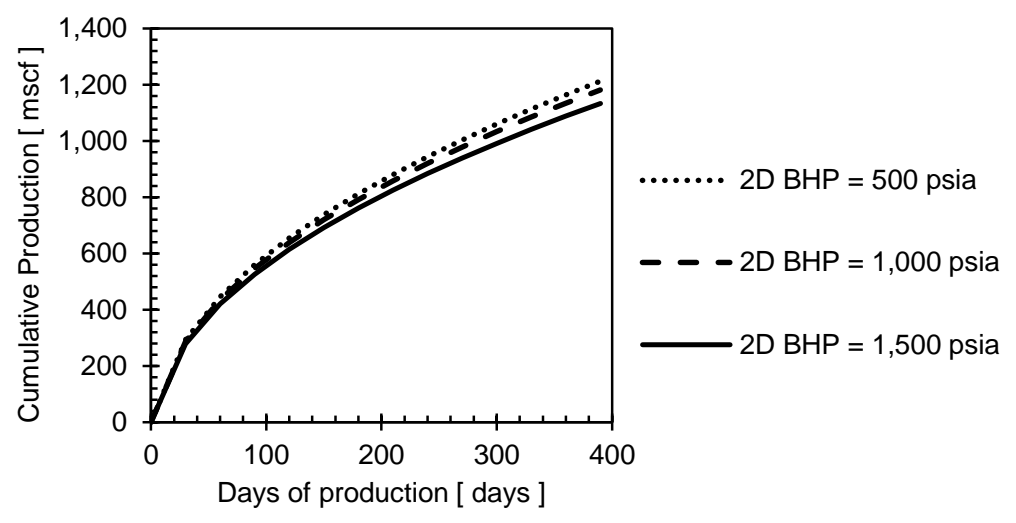

(b) Stress sensitive fracture permeability (steeper gradient)

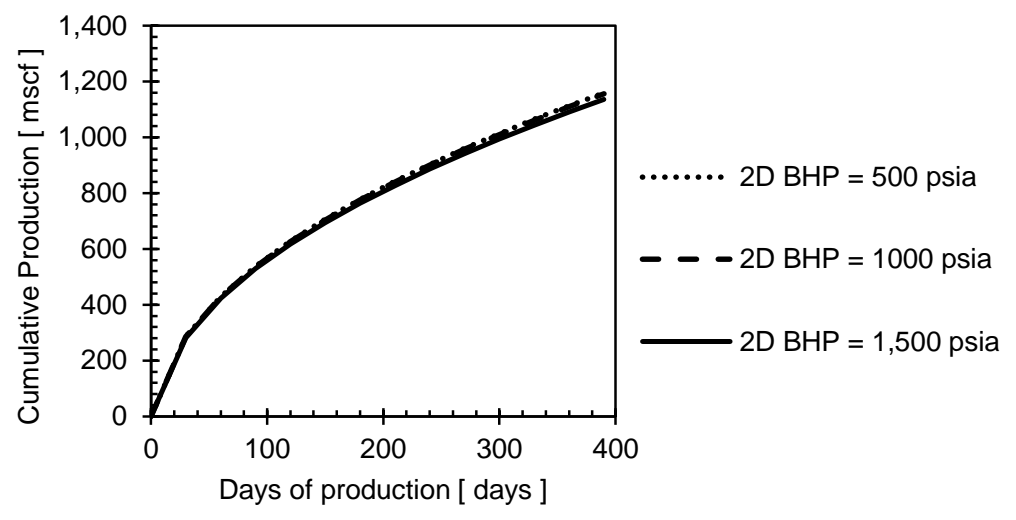

Figure 4.3. Effect of choking with stress sensitive fracture permeability 
Next, the creep effect (permeability decreasing with time) is considered. Bottomhole pressure can change the strain rate because higher bottom-hole pressure will result in less net stress on a fracture. Depending on the sensitivity to net stress, cumulative production could improve. As an example, two cases are carried out. One is with high strain rate, converted to fracture permeability decreasing with production time. The bottom-hole pressure is fixed at 500 psia. The other case is without any deformation in the fracture, therefore no decrease in permeability, and the bottom-hole pressure is fixed at 1,000 psia. The result is shown in Figure 4.4. We observe slightly better cumulative production for the latter case, which could be interpreted as a potential choking effect. However, In this case, the difference is not significant. Moreover, the way how to find out the actual decline rate of fracture conductivity or its sensitivity to stress remains as a huge uncertainty.

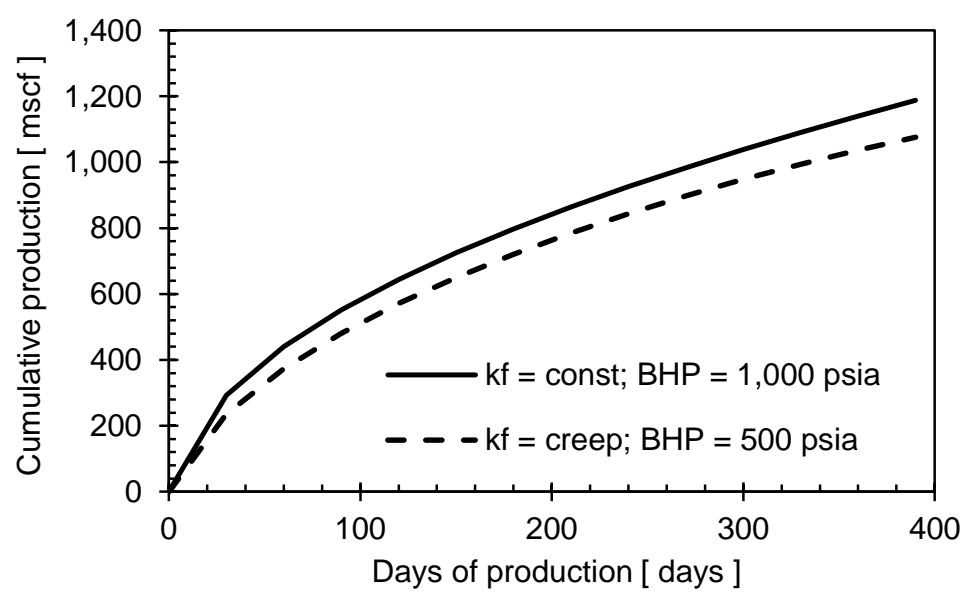

Figure 4.4. Effect of choking with time dependent fracture permeability 


\section{CONCLUSION}

Building a petrophysically consistent apparent permeability model for organic-rich shale was the main focus of this work, and it was built starting from looking into the pore structures in shale matrix. The matrix was split into two parts, one is round shape organic pores and the other is slit-shape pores. Molecular transport effects are considered for the flow in the organic pores, and stress sensitive convection for slit-shape pores. Then a sensitivity study was conducted to investigate the influence of these flow mechanisms on production. Stress sensitive convection is the most influential among the all mechanisms. The effect of molecular transports could be important at lower pore pressure, which can be seen in the region close to a production well or a hydraulic fracture.

The contribution of a hydraulic fracture on the production was also considered. The flow was considered in a continuum scale unlike shale matrix. However, the flow could still be complicated due to its sensitivity to stress or flow rate. These effects on fracture permeability and production was investigated as well, starting the discussion from static permeability. The main observation from this investigation was that there exists a minimum fracture permeability that can realize the production performance of infinite conductivity. The production would be limited below this minimum fracture permeability. This value can be obtained by running several cases of flow simulations with different fracture permeabilities.

A practical problem in the oil field industry were considered using the sector model and the flow simulator. The problem was about the effect of choking a well. We looked 
for a case when higher bottom-hole pressure could result in a better cumulative production. The only possible case was when the fracture permeability declines with time and the decline rate is a function of the bottom-hole pressure.

In summary, according to the formulation of the apparent permeability shown in this work, it is preferable to look for a spot in shale whose matrix permeability is less sensitive to effective stress. In other word, larger $p_{l}$ or $m$ values in Equation (2.28) will give a better production. Once good reservoir quality is located, the next step is to place a hydraulic fracture with high enough conductivity. When we turn to production issue, choking effect might not result in significant increase in cumulative production unless creep effect is significant. 


\section{REFERENCES}

Akkutlu, I.Y., and Fathi, E. 2012. Multi-scale Gas Transport in Shales with Local Kerogen Heterogeneities. SPE Journal, Volume 17 (4), 1002-1011.

Ambrose, R.J., Hartman, R.C., Diaz-Campos, M., Akkutlu, I.Y., and Sondergeld, C.H. 2012. Shale Gas in-place Calculations Part I - New Pore-scale Considerations. SPE Journal, Volume 17 (1), 219-229.

Amini, S., and Valkó, P.P. 2010. Using Distributed Volumetric Sources To Predict Production From Multiple-Fractured Horizontal Wells Under Non-Darcy-Flow Conditions. SPE Journal, Volume 15 (1), 105-115.

Bae, J.S., and Do, D.D. 2003. Surface Diffusion of Strongly Adsorbing Vapors in Activated Carbon by a Differential Permeation Method. Chemical Engineering Science, Volume 58 (19) 4403-4415.

Bui, K., and Akkutlu, I.Y. 2015. Nanopore Wall Effect on Surface Tension of Methane. Journal of Molecular Physics, in print.

Chen, Z., and Zhang, Y. 2009. Well flow models for various numerical methods. Internatinal Journal of Numerical Analysis and Modeling, Volume 6 (3) 375-388.

Do, D.D. 1998. Adsorption Analysis: Equilibria and Kinetics. Imperial College Press, London.

Do, D.D. and Wang, K. 1998. A New Model for the Description of Adsorption Kinetics in Heterogeneous Activated Carbon. Carbon, Volume 36 (10) 1539-1554. 
Fathi, E., and Akkutlu, I.Y. 2009. Matrix Heterogeneity Effects on Gas Transport and Adsorption in Coalbed and Shale Gas Reservoirs. Journal of Transport in Porous Media, Volume 80 (2), 281-304.

Fathi, E., Tinni, A., and Akkutlu, I.Y. 2012. Correction to Klinkenberg Slip Theory for Gas Flow in Nano-capillaries. International Journal of Coal Geology, special issue on Shale, Volume 103, 51-59.

Fredd, C.N., McConnell, S.B., Boney, C.L., and England, K.W. 2001. Experimental Study of Fracture Conductivity for Water-Fracturing and Conventional Fracturing Applications. SPE Journal, Volume 6 (3), 288 - 298.

Gangi, A.F. 1978. Variation of Whole and Fractured Porous Rock Permeability with Confining Pressure. International Journal of Rock Mechanics and Mining Sciences and Geomechanics Abstracts, Volume 15 (5) 249-257.

Heller, R. 2013. Multiscale Investigation of Fluid Transport in Gas Shales. PhD thesis, Stanford University, Stanford, California (September 2013).

Heller, R., and Zoback, M. 2013. Laboratory Measurements of Matrix Permeability and Slippage Enhanced Permeability in Gas Shales. Unconventional Resources Technology Conference, 12-14 August, Denver, Colorado, USA.

Higgs-Palmer Technologies, 2009 Unconventional Resources Characterizing Stimulation Domains, for Improved Well Completions in Gas Shales. (RPSEA Project Number: 09122-02)

Jaeger, J.C., Cook, N.G.W., and Zimmerman, R.W. 2007. Fundamentals of Rock Mechanics. Blackwell Publishing, Malden, MA. 
Javadpour, F., Fisher, D., and Unsworth, M. 2007. Nanoscale Gas Flow in Shale Gas Sediments. Journal of Canadian Petroleum Technology, Volume 46 (10), 55-61. Javadpour, F. 2009. Nanopores and Apparent Permeability of Gas Flow in Mudrocks (Shales and Siltstone). Journal of Canadian Petroleum Technology, Volume 48 (8), 16-21.

Kang, S., Fathi, E., Ambrose, R.J., Akkutlu, I.Y., and Sigal, R.F. 2011. Carbon Dioxide Storage Capacity of Organic-rich Shales. SPE Journal, Volume 16 (4), 842-855.

Karniadakis, G., Beskok, A., and Aluru, N. 2005 Microflows and Nanoflows: Fundamentals and Simulation. Springer, New York.

Kwon, O., Kronenberg, A.K., Gangi, A.F., Johnson, B., and Herbert, B.E. 2004. Permeability of Illite-bearing Shale: 1. Anisotropy and Effects of Clay Content and Loading. Journal of Geophysical Research-Solid Earth, Volume 109 (B10), B10205.

Li, D., and Engler, T.W. 2001. Modeling and Simulation of Non-Darcy Flow in Porous Media. SPE/DOE Improved Oil Recovery Symposium, 13-17 April, Tulsa, Oklahoma.

Loucks, R.G., Reed, R.M., Ruppel, S.C., and Hammes, U. 2012. Spectrum of pore types and networks in mudrocks and a descriptive classification for matrix-related mudrock pores. AAPG Bulletin, Volume 96 (6), 1071-1098.

Loucks, R.G., Reed, R.M., Ruppel, S.C., and Jarvie, D.M. 2009. Morphology, Genesis, and Distribution of Nanometer-Scale Pores in Siliceous Mudstones of the 
Mississippian Barnett Shale. Journal of Sedimentary Research, Volume 79 (12), $848-861$.

Macdonald, I.F., El-Sayed, M.S., Mow, K., and Dullien, F.A.L. 1979. Flow through Porous Media-the Ergun Equation Revisited. Industrial \& Engineering Chemistry Fundamentals, Volume 18 (3), 199-208.

Meyer, B.R., Bazan, L.W., Jacot, R.H., and Lattibeaudiere, M.G. 2010. Optimization of Multiple Transverse Hydraulic Fractures in Horizontal Wellbores. SPE Unconventional Gas Conference, 23-25 February, Pittsburgh, Pennsylvania, USA.

Miskimins, J.L., Lopez, H.D.J., and Barree, R.D. 2005. Non-Darcy Flow in Hydraulic Fractures: Does It Really Matter? SPE Annual Technical Conference and Exhibition, 9-12 October, Dallas, Texas.

Palciauskas, V.V., and Domenico, P.A. 1980. Microfracture development in compacting sediments; relation to hydrocarbon-maturation kinetics. AAPG Bulletin, Volume 64 (6), 927-937.

Riewchotisakul, S. 2015. Effects of Adsorption on Molecular Transport in Nanotube. MSc Thesis, Texas A\&M University, College Station, Texas (May 2015).

Ruth, D., and Ma, H. 1992. On the derivation of the Forchheimer equation by means of the averaging theorem. Transport in Porous Media, Volume 7 (3), 255-264.

Ruthven, D.M. 1984. Principles of Adsorption and Adsorption Processes. Wiley, New York.

Sakhaee-Pour, A., and Bryant, S. 2012. Gas Permeability of Shale. SPE Reservoir Evaluation \& Engineering, Volume 15 (4), 401-409. 
Santos, J.M., and Akkutlu, I.Y. 2013. Laboratory Measurement of Sorption Isotherm under Confining Stress with Pore Volume Effects. SPE Journal, Volume 18 (5), 924-931.

Schwartz, C.W., and Kolluru, S. 1981. Laboratory Investigation Of Creep Of Jointed Rock Material. American Rock Mechanics Association. The 22nd U.S. Symposium on Rock Mechanics (USRMS), 29 June-2 July, Cambridge, Massachusetts.

Schwartz, C.W., and Kolluru, S. 1982. The Influence Of Stress Level On The Creep Of Unfilled Rock Joints. American Rock Mechanics Association. The 23rd U.S Symposium on Rock Mechanics (USRMS), 25-27 August, Berkeley, California.

Walsh, J.B. 1981 Effect of Pore Pressure and Confining Pressure on Fracture Permeability, International Journal of Rock Mechanics and Mining Sciences and Geomechanics Abstracts, Volume 18 (5), 429-435.

Wasaki, A., and Akkutlu, I.Y. 2014. Permeability of Organic-rich Shale. SPE Annual Technical Conference and Exhibition, 27-29 October, Amsterdam, The Netherlands.

Wattenbarger, R.A., El-Banbi, A.H., Villegas, M.E., and Maggard, J.B. 1998. Production Analysis of Linear Flow Into Fractured Tight Gas Wells. SPE Rocky Mountain Regional/Low-Permeability Reservoirs Symposium, 5-8 April, Denver, Colorado. Wen, Q., Zhang, S., Wang, L., Liu, Y., and Li, X. 2007. The effect of proppant embedment upon the long-term conductivity of fractures. Journal of Petroleum Science and Engineering, Volume 55 (3-4), 221-227. 
Zhang, J., Kamenov, A., Hill, A.D., and Zhu, D. 2014. Laboratory Measurement of Hydraulic-Fracture Conductivities in the Barnett Shale. SPE Production \& Operations, Volume 29 (3), $216-227$.

Zimmerman, R.W. 1991. Compressibility of Sandstones. Elsevier, New York. 


\section{APPENDIX A}

\section{A.1. Derivation of the Sorbed-phase Amount}

Sorbed phase amount, $C_{\mu}$, is shown in Equation (A6). The equation is derived by the following steps. According to Ruthven (1984) or Do and Wang (1998), Langmuir kinetics can be described as follows. Here, $R_{a d s}$ in Equation (A1) is the sorption rate and $R_{d e s}$ in Equation (A2) is the desorption rate, both with units in moles/volume/second. $k_{a}$ and $k_{d}$ are the rate constants for sorption and desorption, respectively.

$$
\begin{array}{r}
R_{a d s}=k_{a} p\left(C_{\mu s}-C_{\mu}\right) \\
R_{d e s}=k_{d} C_{\mu} \ldots \ldots
\end{array}
$$

Referring to Equation (A1) and (A2), mass change of sorbed phase can be written as Equation (A3).

$$
\frac{\partial C_{\mu}}{\partial t}=k_{a} p\left(C_{\mu s}-C_{\mu}\right)-k_{d} C_{\mu}
$$

Expression of $C_{\mu}$ that we use in the main body of the paper is obtained by introducing $\partial$

$C_{\mu} \partial t=0$ based on the assumption that sorption and desorption reaches equilibrium instantly, i.e., equilibrium sorption condition is satisfied. From this condition, Equation (A4) could be derived.

$$
\begin{gathered}
0=k_{a} p\left(C_{\mu s}-C_{\mu}\right)-k_{d} C_{\mu} \\
\Leftrightarrow 0=k_{a} p C_{\mu s}-k_{a} p C_{\mu}-k_{d} C_{\mu}
\end{gathered}
$$




$$
\begin{gathered}
\Leftrightarrow\left(k_{a} p+k_{d}\right) C_{\mu}=k_{a} p C_{\mu s} \\
\therefore C_{\mu}=C_{\mu s} \frac{k_{a} p}{k_{a} p+k_{d}} \cdots
\end{gathered}
$$

Here, by introducing $K=k_{d} k_{d}$, then Equation (A4) can be simplified even more, as shown in Equation (A5).

$$
C_{\mu}=C_{\mu s} \frac{K p}{K p+1}
$$

The new variable $K$ is equivalent to the inverse of the Langmuir pressure $p_{L}$, i.e., $\mathrm{K}=1 / p_{L}$ (Do and Wang (1998); Do (1998)). Finally, we obtain the familiar form (A6) as follows.

$$
C_{\mu}=C_{\mu s} \frac{p}{p+p_{L}}
$$

The pressure that appears in Equation (A6) should be the pore pressure in the organic pore, strictly speaking. However, in this paper, we assume pore pressure in organic pores and inorganic pores are the same, for simplicity. 


\section{APPENDIX B}

\section{B.1. Derivation of the Apparent Permeability, Equation (2.6)}

Starting from Equation (2.4), we manipulate the mass flux terms on the right-hand-side (RHS) to obtain apparent permeability. Here, we would like to show the procedure step by step.

$$
\begin{gathered}
(R H S)=\frac{\partial}{\partial x}\left(D_{S} \frac{\partial C_{\mu}}{\partial x}\right)+\frac{\partial}{\partial x}\left(D \frac{\partial C}{\partial x}\right)+\frac{\partial}{\partial x}\left(C \frac{k_{m}}{\mu} \frac{\partial p}{\partial x}\right) \\
\Leftrightarrow(R H S)=\frac{\partial}{\partial x}\left[D_{s} \frac{\partial}{\partial x}\left(C_{\mu s} \frac{p}{p+p_{L}}\right)\right]+\frac{\partial}{\partial x}\left(D \frac{\partial C}{\partial x}\right)+\frac{\partial}{\partial x}\left(C \frac{k_{m}}{\mu} \frac{\partial p}{\partial x}\right) \\
\Leftrightarrow(R H S)=\frac{\partial}{\partial x}\left[D_{s} \frac{\partial}{\partial x}\left(C_{\mu s} \frac{p}{p+p_{L}}\right)+D \frac{\partial C}{\partial x}+C \frac{k_{m}}{\mu} \frac{\partial p}{\partial x}\right]
\end{gathered}
$$

As for the second term, pore diffusion term, free gas moles concentration can be related to gas density, $C=\rho_{g} M$.

$$
\begin{gathered}
\Leftrightarrow(R H S)=\frac{\partial}{\partial x}\left[D_{s} \frac{\partial}{\partial x}\left(C_{\mu s} \frac{p}{p+p_{L}}\right)+D \frac{1}{M} \frac{\partial \rho_{g}}{\partial x}+C \frac{k_{m}}{\mu} \frac{\partial p}{\partial x}\right] \\
\Leftrightarrow(R H S)=\frac{\partial}{\partial x}\left[D_{s} \frac{\partial}{\partial x}\left(C_{\mu s} \frac{p}{p+p_{L}}\right)+D \frac{1}{M} \frac{\rho_{g}}{\rho_{g}} \frac{d \rho_{g}}{d p} \frac{\partial p}{\partial x}+C \frac{k_{m}}{\mu} \frac{\partial p}{\partial x}\right]
\end{gathered}
$$

Defining the gas coefficient of isothermal gas compressibility as $c_{g}=1 / \rho_{g}(\partial \rho g \partial p)$, we obtain Equation (B1):

$$
\Leftrightarrow(R H S)=\frac{\partial}{\partial x}\left[D_{s} \frac{\partial}{\partial x}\left(C_{\mu s} \frac{p}{p+p_{L}}\right)+D \frac{\rho_{g} c_{g}}{M} \frac{\partial p}{\partial x}+C \frac{k_{m}}{\mu} \frac{\partial p}{\partial x}\right]
$$




$$
\begin{gathered}
\Leftrightarrow(R H S)=\frac{\partial}{\partial x}\left[D_{S} \frac{\partial}{\partial x}\left(C_{\mu s} \frac{p}{p+p_{L}}\right)+D C c_{g} \frac{\partial p}{\partial x}+C \frac{k_{m}}{\mu} \frac{\partial p}{\partial x}\right] \\
\therefore(R H S)=\frac{\partial}{\partial x}\left[D_{s} C_{\mu s} \frac{p_{L}}{\left(p+p_{L}\right)^{2}} \frac{\partial p}{\partial x}+D C c_{g} \frac{\partial p}{\partial x}+C \frac{k_{m}}{\mu} \frac{\partial p}{\partial x}\right]
\end{gathered}
$$

The total mass flux $J$ can then be expressed as Equation (B2):

$$
J=\left[D_{S} C_{\mu s} \frac{p_{L}}{\left(p+p_{L}\right)^{2}}+D C c_{g}+C \frac{k_{m}}{\mu}\right] \frac{\partial p}{\partial x}
$$

Then by binding terms which has the same dimension as $k_{m}$, we can come up with a new expression of apparent permeability (Equation (B3)).

$$
k_{\text {gas }}=k_{m}+\mu D c_{g}+\mu D_{s} \frac{C_{\mu s}}{C} \frac{p_{L}}{\left(p+p_{L}\right)^{2}}
$$

Here, if we introduce Equation (2.3) and the definition of free gas mole concentration, $C$ $=p / z R T$, we can eliminate moles concentration expressions. Remember that there is a relationship, $\rho_{s c, g a s} B_{g}=\rho_{g}$, where $B_{g}$ is free gas formation volume factor.

$$
\begin{gathered}
k_{\text {gas }}=k_{m}+\mu D c_{g}+\mu D_{s} \frac{V_{s L} \rho_{s c, \text { gas }} \rho_{\text {grain }}}{\varepsilon_{k s}} \frac{z R T}{M p} \frac{p_{L}}{\left(p+p_{L}\right)^{2}} \\
\Leftrightarrow k_{\text {gas }}=k_{m}+\mu D c_{g}+\mu D_{s} \frac{V_{s L} \rho_{s c, \text { gas }} \rho_{\text {grain }}}{\varepsilon_{k s} \rho_{g}} \frac{p_{L}}{\left(p+p_{L}\right)^{2}} \\
\therefore k_{\text {gas }}=k_{m}+\mu D c_{g}+\mu D_{s} \frac{V_{s L} \rho_{\text {grain }} B_{g}}{\varepsilon_{k s}} \frac{p_{L}}{\left(p+p_{L}\right)^{2}}
\end{gathered}
$$




\section{APPENDIX C}

\section{C.1. Derivation of Gangi's Permeability Model}

The derivation comes from deriving two relationships assuming the following configuration. One is the relationship between permeability and slit width assuming Hagen-Poiseuille type of flow for Newtonian fluid. The other part is the relation between slit width and net stress. By coupling these two relationships, Gangi (1978) derived the relation between net stress and permeability.

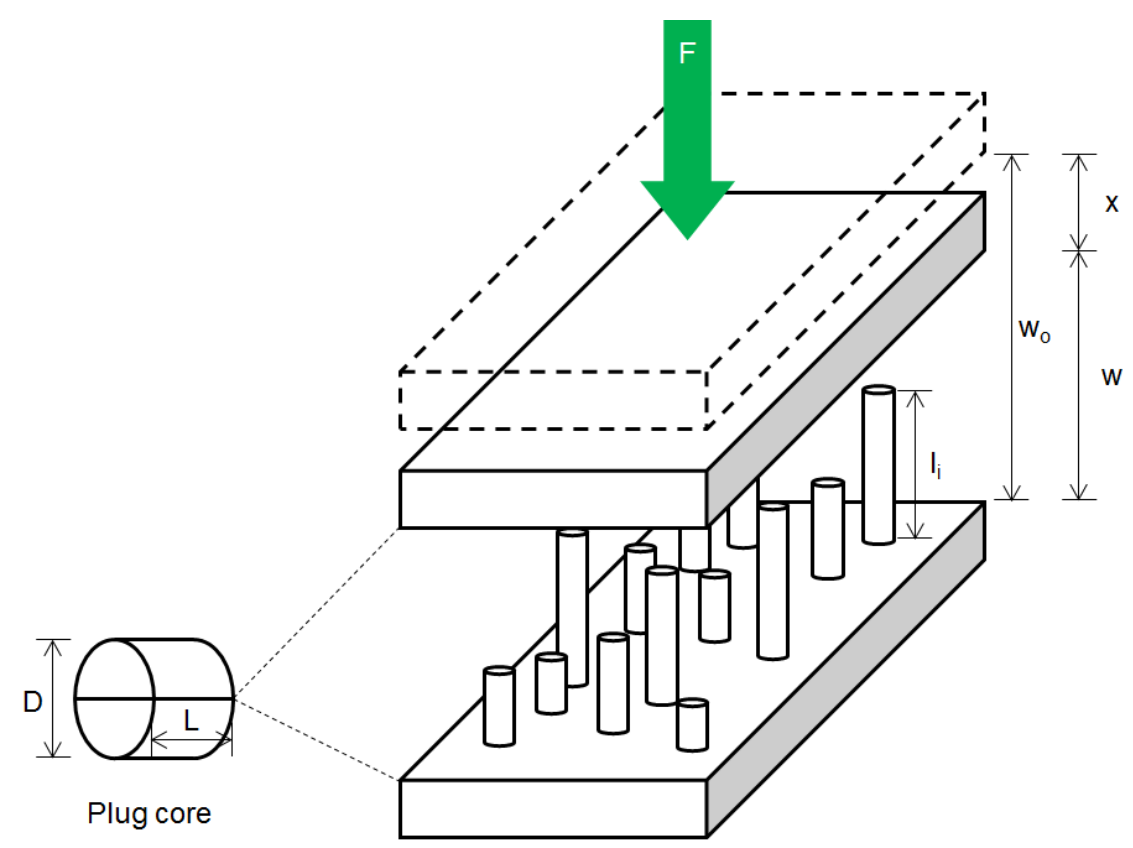

Figure C.1. Conceptual image of "Bed of Nails" model [modified from Gangi (1978)] 


\section{C.2. Relationship between Permeability of the System and Slit Width}

A flow between two planes is shown as follows, assuming a flow for Newtonian fluid. Here $w$ is the width between the planes, $\mu$ is the viscosity of the fluid, $d p / d x$ is the pressure gradient within the flow path, and $u_{\text {ave }}$ is the average fluid flow velocity across the width.

$$
u_{\text {ave }}=\frac{w^{2}}{12 \mu} \frac{d p}{d x}
$$

Comparison with Darcy's velocity, $v$, the relation between $u_{\text {ave }}$ and $v$ could be shown as $\phi u_{\text {ave }}=v . \phi$ is the porosity assuming the flow occurs only in the slit between the halfcut core in Figure C.1. Therefore, $\phi=4 w /(\pi D)$ and Darcy's velocity, $v$, could be shown as follows.

$$
v=\phi u_{\text {ave }}=\frac{w^{3}}{3 \pi D} \frac{1}{\mu} \frac{d p}{d x}
$$

Therefore, the permeability of this core when a flow through this cylindrical core is applied could be written as follows.

$$
k=\frac{w^{3}}{3 \pi D}
$$

In case, the derivation of Equation $(\mathrm{C} 1)$ is explained further here. Think about a flow between two planes as shown in Figure C.2, and build the following force balance equation. This leads to Equation (C5). Here, $\tau$ is the shear stress at the plane.

$$
\begin{gathered}
\tau_{w} 2 L h=\Delta P w h \\
\tau_{w}=\frac{w}{2} \frac{\Delta P}{L} \cdots
\end{gathered}
$$



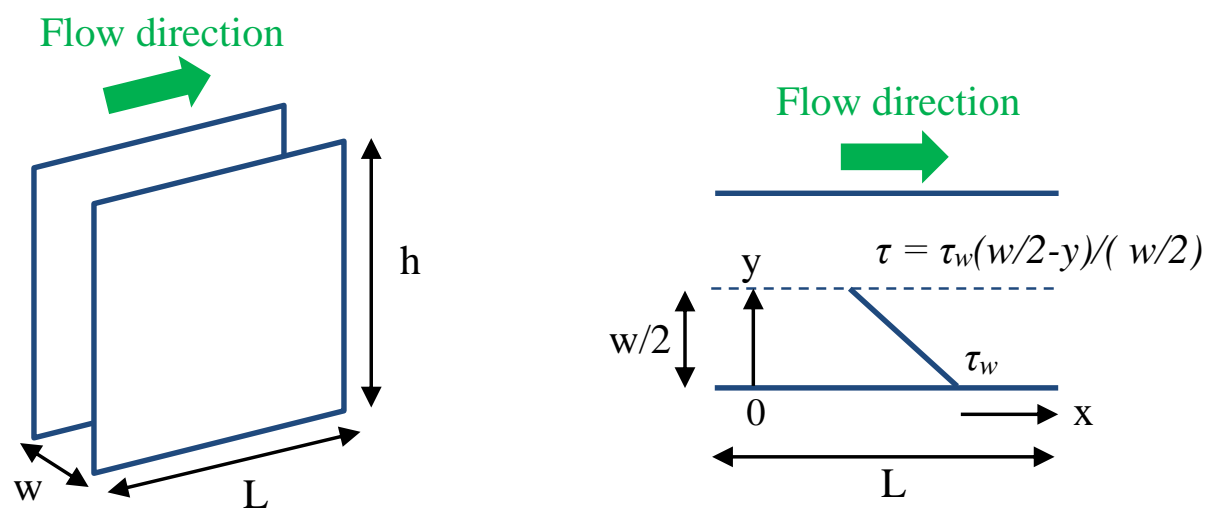

Figure C.2. A flow between two planes

Assume the distribution of shear stress across the width is linear, which shows maximum value at the wall, decreases as it becomes far from the wall and reaches zero at the center of the flow $(y=w / 2)$. Then the shear stress could be written as follows.

$$
\tau(y)=\tau_{w} \frac{w / 2-y}{w / 2}
$$

And according to the definition of shear stress, the following equation is true for Newtonian fluid. Here, $u$ is the velocity in $x$-direction at location $y$.

$$
\tau(y)=\mu \frac{\partial u}{\partial y}
$$

Using Equation (C5), $\tau_{w}$ could be eliminated from Equation (C6), then coupled with Equation (C7), $\tau$ could be eliminated next. Finally we could obtain Equation (C1) by assuming $d p / d x=\Delta P / L$. 


\section{C.3. Relationship between Net Stress and Slit Width}

First, a force balance equation for the force, $F$, on the bed of nails assuming an elastic body for the nails. Here, $I_{o}$ is the total number of the nails, and $k$ is a spring constant. Since the material is the same, a uniform spring constant is applied for all nails. Equation (C8) is constructed based on the Hook's law.

$$
\begin{gathered}
F=\sum_{i=1}^{I_{o}} k R\left(x+l_{i}-w_{o}\right) \\
R(x)= \begin{cases}x & (x>0) \\
0 & (x \leq 0)\end{cases}
\end{gathered}
$$

The spring constant, $k$, in Equation (C8) can be shown using Young's Modulus, $E$, by comparing Hook's law $\left(F_{i}=k x_{i}\right)$ and the definition Young's Modulus $\left(\sigma_{i}=E \varepsilon_{i}\right)$ since $\sigma_{\mathrm{i}}=$ $F_{i} / a_{i}$ and $\varepsilon_{i}=x_{i} / l_{i} . a_{i}$ is the cross-sectional area of a nail.

$$
k=E \frac{a_{i}}{l_{i}}
$$

Since the left hand side of Equation (C10) is constant, $a_{i} / l_{i}$ is also constant. For convenience, introduce a constant $b$ and define it as follows.

$$
\frac{a_{i}}{l_{i}}=b w_{o}
$$

Then Equation (C8) can be reshaped.

$$
F=E b w_{o} \sum_{i=1}^{I_{o}} R\left(x+l_{i}-w_{o}\right)
$$

Here, the plane area of the bed is $A$, then the force $F$ could be replaced with net stress $p_{e}(=F / A)$. Also introduce a function, $n\left(l_{i}\right)$, which is a number of nails with the same 102 
length $l_{i}$. Then reshape Equation $(\mathrm{C} 12)$ again. Here, $N_{j}$ is the number of the total number of the nail sizes.

$$
p_{e}=\frac{E b w_{o}}{A} \sum_{i=1}^{N_{j}} n\left(l_{j}\right) R\left(x+l_{j}-w_{o}\right)
$$

Since $N_{j}$ is a very large number, Equation $(\mathrm{C} 13)$ could be shown in a form of integral.

$$
p_{e}=\frac{E b w_{o}}{A} \int_{l_{\min }}^{l_{\max }} n(l) R\left(x+l-w_{o}\right) \mathrm{d} l
$$

Here, introduce another parameter called shortness, $\xi\left(=w_{o}-l\right)$ then we could obtian Equation (C15).

$$
p_{e}=\frac{E b w_{o}}{A} \int_{\xi_{\min }}^{\xi_{\max }} n(\xi) R(x-\xi) \mathrm{d} \xi
$$

Here, introduce another function, $N(\xi)$, which is a number of the nails that lies between $0 \sim \xi$. Using this function, reshape Equation (C15).

$$
\begin{array}{r}
\mathrm{d} N(\xi)=n(\xi) \mathrm{d} \xi \ldots \ldots \ldots . . . \\
p_{e}=\frac{E b w_{o}}{A} \int_{0}^{N\left(\xi_{\max }\right)} R(x-\xi) \mathrm{d} N(\xi) \\
=\frac{E b w_{o}}{A} \int_{0}^{N(x)}(x-\xi) \mathrm{d} N(\xi)
\end{array}
$$

Now, suppose a power law distribution for the nails shortness for mathematical simplicity reason.

$$
N(\xi)=I_{o}\left(\frac{x}{w_{o}}\right)^{n-1} \quad(1 \leq n \leq \infty)
$$




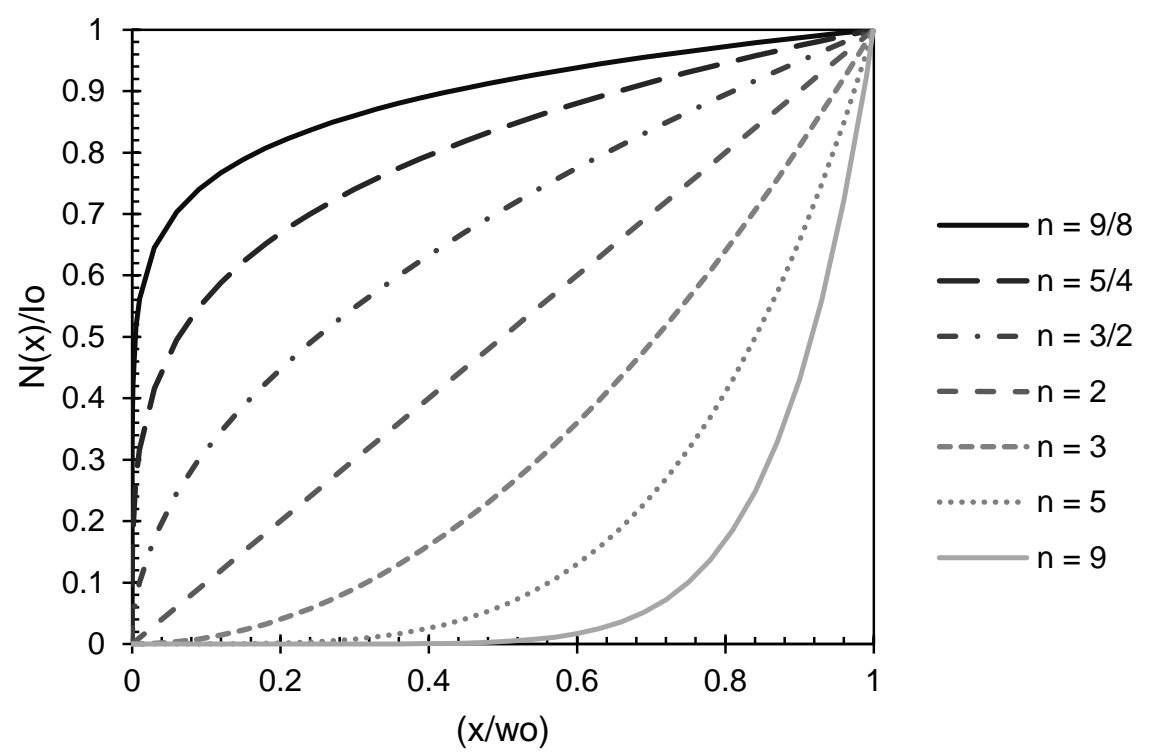

Figure C.3. The power law distribution of the nails

Here, recall $x=w_{o}-w$, then Equation (C17) coupled with Equation (C18) could be shown as follows.

$$
p_{e}=\frac{E b w_{o}^{2} I_{o}}{n A}\left(\frac{w_{o}-w}{w_{o}}\right)^{n}
$$

Replace $E b w_{o}^{2} I_{o} /(n A)$ with $p_{1}$ and $1 / n$ with $m$ gives the following equation.

$$
\left(\frac{p_{e}}{p_{1}}\right)^{m}=1-\left(\frac{w}{w_{o}}\right)
$$

Combine Equation (C3) and (C20) then we could derive the Gangi's permeability model. Another way to relate $p_{1}$ is probably to connect it to a Sneddon solution of symmetric line crack with uniform pressure condition. 